\title{
DIFFERENTIAL FORMS ON LOG CANONICAL SPACES
}

\author{
DANIEL GREB, STEFAN KEBEKUS, SÁNDOR J KOVÁCS, AND THOMAS PETERNELL
}

In memory of Eckart Viehweg

\begin{abstract}
The present paper is concerned with differential forms on log canonical varieties. It is shown that any $p$-form defined on the smooth locus of a variety with canonical or klt singularities extends regularly to any resolution of singularities. In fact, a much more general theorem for log canonical pairs is established. The proof relies on vanishing theorems for log canonical varieties and on methods of the minimal model program. In addition, a theory of differential forms on dlt pairs is developed. It is shown that many of the fundamental theorems and techniques known for sheaves of logarithmic differentials on smooth varieties also hold in the dlt setting.

Immediate applications include the existence of a pull-back map for reflexive differentials, generalisations of Bogomolov-Sommese type vanishing results, and a positive answer to the Lipman-Zariski conjecture for klt spaces.
\end{abstract}

\section{CONTENTS}

PART I. INTRODUCTION

1. Introduction

2. Notation, conventions and standard facts 5

3. Examples

PART II. APPLICATIONS OF THE EXTENSION THEOREM

4. Pull-back morphisms for reflexive differentials

5. Reflexive differentials on rationally chain connected spaces 15

6. The Lipman-Zariski Conjecture for klt spaces 15

7. Bogomolov-Sommese type results on log canonical spaces 16

PART III. REFLEXIVE FORMS ON DLT PAIRS

8. Overview and main results of Part III

9. The local structure of dlt pairs in codimension 2

10. Relative differential sequences on dlt pairs 26

11. Residue sequences for reflexive differential forms 30

12. The residue map for 1 -forms

Date: November 1, 2011.

Daniel Greb was supported in part by an MSRI postdoctoral fellowship during the 2009 special semester in Algebraic Geometry. Stefan Kebekus and Thomas Peternell were supported in part by the DFG-Forschergruppe "Classification of Algebraic Surfaces and Compact Complex Manifolds". Sándor Kovács was supported in part by NSF Grants DMS-0554697 and DMS-0856185, and the Craig McKibben and Sarah Merner Endowed Professorship in Mathematics.

A shortened version of this paper without color figures has appeared in Publications mathématiques de l'IHÉS, DOI:10.1007/s10240-011-0036-0 The final publication is available at www.springerlink.com 
PART IV. COHOMOLOGICAL METHODS

13. Vanishing results for pairs of Du Bois spaces 36

14. Steenbrink-type vanishing results for log canonical pairs 39

15. Generic base change for cohomology with supports 41

PART V. EXTENSION WITH LOGARITHMIC POLES 4

16. Main result of this part

17. Proof of Theorem 16.2

\begin{tabular}{llll} 
PART VI. & PROOF OF THE EXTENSION THEOREM 1.5 & 51 \\
\hline
\end{tabular}

18. Proof of Theorem 1.5, idea of Proof

19. Proof of Theorem 1.5, overview of the proof 53

20. Step 0 in the proof of Theorem $1.5 \quad 55$

21. Step 1 in the proof of Theorem 1.5

22. Step 2 in the proof of Theorem $1.5 \quad 56$

23. Step 3 in the proof of Theorem $1.5 \quad 57$

24. Step 4 in the proof of Theorem $1.5 \quad 58$

25. Step 5 in the proof of Theorem 1.5

$\begin{array}{llll}\text { PART VII. Appendix } & 64\end{array}$

Appendix A. Effective linear combinations of exceptional divisors $\quad 64$

\begin{tabular}{ll|l|}
\hline Appendix B. Finite group actions on coherent sheaves & 65 \\
\hline
\end{tabular}

$\begin{array}{lll}\text { Appendix C. The Four-Lemmas for vector spaces } & 68\end{array}$

\begin{tabular}{l|l} 
References & 68 \\
\hline
\end{tabular}

List of Figures

List of Tables

\section{PART I. INTRODUCTION}

\section{INTRODUCTION}

Differential forms play an essential role in the study of algebraic varieties. On a smooth complex variety $X$ of dimension $n$ the sheaf $\omega_{X}=\Omega_{X}^{n}$ of $n$-forms is of particular importance as it appears both in Serre duality and in the Kodaira vanishing theorem. As observed by Grauert and Riemenschneider, these two roles do not generalise the same way to the singular case. If $X$ is singular, there are several possible definitions for the sheaf of $n$-forms, depending on which of the properties one would like to keep. In general, there is one definition that preserves the role of differentials in duality theory and another one suitable for vanishing theorems.

A simple case. Consider the case when $X$ is normal and Gorenstein. In this setting the dualising sheaf $\omega_{X}$ is locally free, and Serre duality holds the same way as in the smooth case. In contrast, the Kodaira vanishing theorem fails in general. There exist a Gorenstein variety $X$ with ample line bundle $\mathscr{L} \in \operatorname{Pic} X$ such that $H^{1}\left(X, \omega_{X} \otimes \mathscr{L}\right) \neq 0$, [GR70, Sect. 3.3]. However, when $\pi: \widetilde{X} \rightarrow X$ is a resolution of singularities and $\widetilde{\omega}_{X}:=\pi_{*} \omega_{\tilde{X}}$, then there exists an inclusion $\widetilde{\omega}_{X} \subseteq \omega_{X}$, the subsheaf $\widetilde{\omega}_{X}$ is independent of the resolution, and Kodaira vanishing holds for $\widetilde{\omega}_{X}$ by [GR70, Thm. 2.1]. Consequently, there are two 
sheaves on $X$ that generalise the notion of the canonical line bundle of a smooth variety: $\omega_{X}$ works for duality, $\widetilde{\omega}_{X}$ for vanishing.

Given the importance of duality and vanishing theorems in complex algebraic geometry, the following question seems natural in this context.

Question 1.1. Given a normal Gorenstein variety $X$, when do the sheaves $\omega_{X}$ and $\widetilde{\omega}_{X}$ agree?

To answer this question, recall that $\omega_{X}$ is locally free and therefore reflexive. If $U \subseteq X$ is any open subset, to give a section $\tau \in \omega_{X}(U)$, it is therefore equivalent to give an $n$-form on the smooth locus of $U$. In other words, to give a section $\tau \in \omega_{X}(U)$, it is equivalent to give an $n$-form $\tau^{\prime} \in \omega_{\tilde{X}}\left(\pi^{-1}(U) \backslash E\right)$, where $E \subset \tilde{X}$ is the exceptional locus of the resolution map $\pi$. In contrast, a section $\sigma \in \widetilde{\omega}_{X}(U)$ is, by definition, an $n$-form $\sigma^{\prime} \in \omega_{\widetilde{X}}\left(\pi^{-1}(U)\right)$.

In summary, we obtain the following equivalent reformulation of Question 1.1.

Question 1.2. When is it true that any $n$-form, defined on an open set of the form $\pi^{-1}(U) \backslash$ $E \subset \widetilde{X}$ extends across $E$, to give a form on $\pi^{-1}(U)$ ?

The answer to Question 1.2 is almost a tautology: it follows directly from the definition that $X$ has canonical singularities if and only if any $n$-form $\pi^{-1}(U) \backslash E$ extends across $E$. The fact that spaces with canonical singularities have a single sheaf that works for both duality and vanishing is one of the reasons for their importance in higher dimensional algebraic geometry.

Main result of this paper. This paper aims to answer Question 1.2 for differential forms of degree $p$, where $p \leq n$ and where $X$ is not necessarily Gorenstein. The main results, formulated in Theorems 1.4 and 1.5 below, assert that if $X$ is log terminal, then any $p$ form will extend. Our results also hold in the logarithmic setup, for log canonical pairs. Immediate applications concern vanishing theorems and other properties of differential forms on $\log$ canonical varieties.

Formulation using reflexive sheaves. Extension properties of differential forms can be expressed in terms of reflexivity of push-forward sheaves. Although perhaps not quite intuitive at first sight, this language is technically convenient. The following observation relates reflexivity and extension properties and will be used throughout the paper.

Observation 1.3. Let $X$ be a normal variety, and $\pi: \widetilde{X} \rightarrow X$ a resolution of singularities, with exceptional set $E \subset \widetilde{X}$. If $\mathscr{A}$ is any locally free sheaf on $\widetilde{X}$, then $\pi_{*} \mathscr{A}$ is torsion free, but not necessarily reflexive. Using that $\operatorname{codim}_{X} \pi(E) \geq 2$, observe that $\pi_{*} \mathscr{A}$ reflexive if and only if any section of $\left.\pi_{*} \mathscr{A}\right|_{X \backslash \pi(E)}$ extends to $X$. Equivalently, $\pi_{*} \mathscr{A}$ is reflexive if and only if any section of $\mathscr{A}$, defined on an open set of the form $\pi^{-1}(U) \backslash E$ extends to $\pi^{-1}(U)$.

1.A. Main results. The main result of this paper gives necessary and sufficient conditions that guarantee reflexivity of $\pi_{*} \Omega_{\widetilde{X}}^{p}$ for all $p \leq \operatorname{dim} X$. Equivalently, the main result gives necessary and sufficient conditions to guarantee that any differential $p$-form on $\widetilde{X}$, defined away from the exceptional set $E$ extends across $E$. The simplest form of our main result is the following.

Theorem 1.4 (Extension theorem for differential forms on klt varieties). Let $X$ be a complex quasi-projective variety with at most klt (Kawamata log terminal) singularities and $\pi: \widetilde{X} \rightarrow X$ a log resolution. Then $\pi_{*} \Omega_{\widetilde{X}}^{p}$ is reflexive for all $p \leq \operatorname{dim} X$. 
Remark 1.4.1. Gorenstein klt varieties have canonical singularities. The statement of Theorem 1.4therefore includes the results discussed in the introduction.

In fact, we prove much more. Our main result works in the category of log canonical (lc) pairs.

Theorem 1.5 (Extension theorem for differential forms on lc pairs). Let $X$ be a complex quasi-projective variety of dimension $n$ and let $D$ be $a \mathbb{Q}$-divisor on $X$ such that the pair $(X, D)$ is log canonical. Let $\pi: \widetilde{X} \rightarrow X$ be a log resolution with $\pi$-exceptional set $E$ and

$$
\widetilde{D}:=\text { largest reduced divisor contained in } \operatorname{supp} \pi^{-1} \text { (non-klt locus), }
$$

where the non-klt locus is the smallest closed subset $W \subset X$ such that $(X, D)$ is klt away from $W$. Then the sheaves $\pi_{*} \Omega_{\widetilde{X}}^{p}(\log \widetilde{D})$ are reflexive, for all $p \leq n$.

Remark 1.5.1. In Section 3 we gathered a number of examples to illustrate Theorem 1.5 and to show that its statement is sharp.

Remark 1.5.2. The name "extension theorem" is justified by Observation 1.3, which asserts that the sheaf $\pi_{*} \Omega_{\widetilde{X}}^{p}(\log \widetilde{D})$ is reflexive if and only if for any open set $U \subseteq X$ and any number $p$, the restriction morphism

$$
H^{0}\left(U, \pi_{*} \Omega_{\widetilde{X}}^{p}(\log \widetilde{D})\right) \rightarrow H^{0}\left(U \backslash \pi(E), \Omega_{X}^{p}(\log \lfloor D\rfloor)\right)
$$

is surjective. In other words, logarithmic $p$-forms defined on the non-singular part of $X$ can be extended to any resolution of singularities.

Remark 1.6. A pair is log canonical if its sheaf of logarithmic $n$-forms satisfies certain conditions, closely related to extension properties. For such pairs, Theorem 1.5 asserts that analogous extension properties hold for forms of arbitrary degrees. This matches the philosophy that the geometry of a variety is governed by the behaviour of its $n$-forms.

1.B. Previous results. The extension problem has been studied in the literature, mostly asking extension only for special values of $p$. For a variety $X$ with only isolated singularities, reflexivity of $\pi_{*} \Omega_{\widetilde{X}}^{p}$ was shown by Steenbrink and van Straten for $p \leq \operatorname{dim} X-2$ without any further assumption on the nature of the singularities, [SvS85, Thm. 1.3]. Flenner extended these results to normal varieties, subject to the condition that $p \leq$ $\operatorname{codim} X_{\text {sing }}-2$, [Fle88]. Namikawa proved reflexivity for $p \in\{1,2\}$, in case $X$ has canonical Gorenstein singularities, [Nam01, Thm. 4]. In the case of finite quotient singularities similar results were obtained in [dJS04]. For a log canonical pair with reduced boundary divisor, the cases $p \in\{1, \operatorname{dim} X-1, \operatorname{dim} X\}$ were settled in [GKK10, Thm. 1.1].

A related setup where the pair $(X, D)$ is snc, and where $\pi: \widetilde{X} \rightarrow X$ is the composition of a finite Galois covering and a subsequent resolution of singularities has been studied by Esnault and Viehweg. In [EV82] they obtain in their special setting a result similar to Theorem 1.5 and additionally prove vanishing of higher direct image sheaves.

We would also like to mention the paper [Bar78] where differential forms are discussed even in non-normal settings.

1.C. Applications. In order to keep the length of this article reasonable, we only give a few applications. These include the existence of a pull-back map for reflexive differentials, rational connectivity of klt spaces, the Lipman-Zariski-conjecture, and BogomolovSommese type results. Many more applications, e.g., to rational connectivity, KodairaAkizuki-Nakano vanishing type results and varieties with trivial canonical classes, will be published separately. 
1.D. Further results of this paper. Apart from the extension results, we develop a theory of differential forms on dlt pairs, showing that many of the fundamental theorems and techniques known for sheaves of logarithmic differentials on smooth varieties also hold in the dlt setting. In particular, there is a satisfactory theory of relative differentials and a residue theory. A detailed introduction is given in Section 8 on page 19.

We believe that these results are of independent interest. Sheaves of reflexive differentials on singular spaces appear naturally when one uses minimal model theory to study compactifications of moduli spaces, where differentials can often be constructed using Hodge-theoretic methods, cf. [VZ02, Vie10]. For a concrete example, we refer to [KK10a] where a study of reflexive differentials on dlt spaces was an important ingredient in a generalisation of Shafarevich hyperbolicity.

1.E. Outline of the paper. The proof of our main theorem is given in two steps. We first extend up to logarithmic poles and then we prove the stronger extension result. This is done in Parts $\nabla$ and VI respectively.

After a preliminary section, mainly devoted to setting up the basic notation, we first give in Part $\amalg$ some applications of the Extension Theorem 1.5. Parts $\amalg$ and IV consist of indispensable technical preparations which might, however, merit attention on their own. In particular, Part ஹpresents a systematic treatment of reflexive differential on dlt pairs. Part IV presents two vanishing theorems for direct image sheaves on log canonical pairs, one of them generalising and expanding Steenbrink's vanishing theorem. A technical vanishing theorem for cohomology with support is also included. In the Appendix $\mathrm{A}$ and $\mathrm{B}$ we present several important facts that are likely known to experts, but for which we were unable to find complete references.

Acknowledgements. The main ideas that led to this paper were perceived when all four authors visited the MSRI special program in algebraic geometry in the spring of 2009. We would like to thank the institute for support and for the excellent working conditions. The work on this paper benefited from discussions with V. Alexeev, C. Birkar, H. Esnault, T. de Fernex, G.M. Greuel, Y. Kawamata, J. Kollár, J. McKernan, M. Reid, O. Riemenschneider, and W. Soergel. The authors want to thank the referee for very valuable remarks and suggestions.

\section{NOTATION, CONVENTIONS AND STANDARD FACTS}

The results of this paper are formulated and proven using the language of higher dimensional algebraic geometry. While most of the definitions and much of the notation we use is fairly standard in the field, we are aware of several instances where definitions have evolved with time and are not always coherently used in the literature. To minimise the potential for confusion, we have chosen to prepend this paper with the present section that collects standard facts and specifies notation wherever misunderstandings seem likely. We quote standard references where possible.

2.A. Base field, Kähler differentials. Throughout the paper, we will work over the field of complex numbers. For a point on a scheme or complex analytic space, $p \in X$, the residue field of $p$ will be denoted by $\kappa(p)$.

The central objects in this paper are differential forms on singular spaces. Traditionally that means (logarithmic) Kähler differentials: If $X$ is a scheme or complex space and $D$ a reduced Weil divisor on $X$ then we denote the sheaves of Kähler differentials (resp. logarithmic Kähler differentials) by $\Omega_{X}^{1}$ (resp. $\Omega_{X}^{1}(\log D)$ ). For a $p \in \mathbb{N}$ we let $\Omega_{X}^{p}=$ $\bigwedge^{p} \Omega_{X}^{1}$ and $\Omega_{X}^{p}(\log D)=\bigwedge^{p} \Omega_{X}^{1}(\log D)$. In particular, $\Omega_{X}^{0}=\Omega_{X}^{0}(\log D)=\mathscr{O}_{X}$. 
Remark 2.1. The sheaves of Kähler differentials do not behave well near singular points. It is often more advantageous to work with their reflexive hulls. See Subsection 2.D for definitions and remarks regarding reflexive differential forms.

2.B. Pairs. The main results of this paper concern pairs of algebraic varieties and effective divisors, which have long been central objects in higher dimensional algebraic geometry. In our discussion of pairs, we follow the language and notational conventions of the book [KM98]. We recall the most important conventions for the reader's convenience.

Definition 2.2 (Pairs and reduced pairs). A pair (or log variety) $(X, D)$ consists of a normal quasi-projective variety $X$ and a boundary, i.e., an effective $\mathbb{Q}$-Weil divisor $D=\sum d_{i} D_{i}$ on $X$ such that $D_{i}$ are reduced effective (integral) Weil-divisors and $d_{i} \in[0,1] \cap \mathbb{Q}$. A reduced pair is a pair $(X, D)$ such that $D$ is reduced, that is, $D=\lfloor D\rfloor$, or equivalently all components of $D$ appear with coefficient 1.

Notation 2.3 (Singularities of pairs). Given a pair $(X, D)$, we will use the notions lc (log canonical), klt, dlt without further explanation or comment and simply refer to [KM98, Sect 2.3] for a discussion and for their precise definitions.

Definition 2.4 (Snc pairs [KM98, 0.4(8)]). Let $(X, D)$ be a pair, and $x \in X$ a point. We say that $(X, D)$ is snc at $x$ if there exists a Zariski-open neighbourhood $U$ of $x$ such that $U$ is smooth and such that $\operatorname{supp}(D) \cap U$ is either empty, or a divisor with simple normal crossings. The pair $(X, D)$ is called snc if it is snc at every point of $X$.

Given a pair $(X, D)$, let $(X, D)_{\text {reg }}$ be the maximal open set of $X$ where $(X, D)$ is snc, and let $(X, D)_{\text {sing }}$ be its complement, with the induced reduced subscheme structure.

Remark 2.5. If $(X, D)$ is a pair, then by definition $X$ is normal. Furthermore, near a general point of $D$, both $X$ and $D$ are smooth. In particular, $\operatorname{codim}_{X}(X, D)_{\text {sing }} \geq 2$.

Example 2.6. In Definition 2.4 it is important that we work in the Zariski topology. If $X=\mathbb{P}^{2}$ and $D \subset X$ is a nodal cubic curve with singular point $x \in D$, then $(X, D)$ is not snc. In particular, $(X, D)_{\mathrm{reg}}=X \backslash\{x\}$.

While snc pairs are the logarithmic analogues of smooth spaces, snc morphisms, which we discuss next, are the analogues of smooth maps. Although relatively snc divisors have long been used in the literature, cf. [Del70, Sect. 3], we are not aware of a good reference that discusses them in detail, so that we include a full definition here.

Notation 2.7 (Intersection of boundary components). Let $(X, D)$ be a pair, where the boundary divisor $D$ is written as a sum of its irreducible components $D=\alpha_{1} D_{1}+\ldots+$ $\alpha_{n} D_{n}$. If $I \subseteq\{1, \ldots, n\}$ is any non-empty subset, we consider the scheme-theoretic intersection $D_{I}:=\cap_{i \in I} D_{i}$. If $I$ is empty, set $D_{I}:=X$.

Remark 2.8 (Description of snc pairs). In the setup of Notation 2.7, it is clear that the pair $(X, D)$ is snc if and only if all $D_{I}$ are smooth and of codimension equal to the number of defining equations: $\operatorname{codim}_{X} D_{I}=|I|$ for all $I$ where $D_{I} \neq \emptyset$.

Definition 2.9 (Snc morphism, relatively snc divisor, [VZ02, Def. 2.1]). If $(X, D)$ is an snc pair and $\phi: X \rightarrow T$ a surjective morphism to a smooth variety, we say that $D$ is relatively snc, or that $\phi$ is an snc morphism of the pair $(X, D)$ if for any set I with $D_{I} \neq \emptyset$ all restricted morphisms $\left.\phi\right|_{D_{I}}: D_{I} \rightarrow T$ are smooth of relative dimension $\operatorname{dim} X-\operatorname{dim} T-|I|$.

Remark 2.10 (Fibers of an snc morphisms). If $(X, D)$ is an snc pair and $\phi: X \rightarrow T$ is any surjective snc morphism of $(X, D)$, it is clear from Remark 2.8 that if $t \in T$ is any point, with preimages $X_{t}:=\phi^{-1}(t)$ and $D_{t}:=D \cap X_{t}$ then the pair $\left(X_{t}, D_{t}\right)$ is again snc. 
Remark 2.11 (All morphisms are generically snc). If $(X, D)$ is an snc pair and $\phi: X \rightarrow T$ is any surjective morphism, it is clear from generic smoothness that there exists a dense open set $T^{\circ} \subseteq T$, such that $D \cap \phi^{-1}\left(T^{\circ}\right)$ is relatively snc over $T^{\circ}$.

2.C. Strong log resolutions. Resolutions of singularities have been in constant use in algebraic geometry ever since Hironaka's seminal work [Hir62]. There are several incompatible definitions of "log resolutions" used in the literature, all serving different purposes. In this paper, we use two variations of the resolution theme, called "log resolution" and "strong log resolution", respectively. We refer to [KM98, p. 3] for further explanations concerning these notions.

Definition 2.12 (Log resolution and strong log resolution [KM98, 0.4(10)]). A log resolution of a pair $(X, D)$ is a surjective birational morphism $\pi: \widetilde{X} \rightarrow X$ such that

(2.12.1) the space $\widetilde{X}$ is smooth,

(2.12.2) the $\pi$-exceptional set $\operatorname{Exc}(\pi)$ is of pure codimension one, and

(2.12.3) the set $\pi^{-1}(\operatorname{supp} D) \cup \operatorname{Exc}(\pi)$ is a divisor with simple normal crossings.

A $\log$ resolution $\pi$ is called a strong $\log$ resolution of $(X, D)$ if the following property holds in addition.

(2.12.4) The rational map $\pi^{-1}$ is a well-defined isomorphism over the open set $(X, D)_{\mathrm{reg}}$.

Fact 2.13 (Hironaka's theorem on resolutions, cf. [Kol07]). Log resolutions and strong log resolutions exist.

Remark 2.14. Let $(X, D)$ be a pair, and $\pi: \widetilde{X} \rightarrow X$ a strong $\log$ resolution. If $D^{\prime} \subseteq D$ is a subdivisor, it is not generally true that $\pi$ is also a strong log resolution of the pair $\left(X, D^{\prime}\right)$.

For an example, let $X=\mathbb{P}^{2}$, let $D \subset \mathbb{P}^{2}$ be a cuspidal plane cubic, and $D^{\prime}=\emptyset$. Let $\pi: \widetilde{X} \rightarrow X$ be a strong log resolution of the pair $(X, D)$. Since $(X, D)$ is not snc, the morphism $\pi$ is not isomorphic. On the other hand, since $\left(X, D^{\prime}\right)$ is snc, the property (2.12.4) of Definition 2.12 asserts that any strong log resolution of $\left(X, D^{\prime}\right)$ must in fact be isomorphic.

The following elementary lemma shows that the property (2.12.4) is the only property that possibly fails when one replaces $D$ by a smaller divisor.

Lemma 2.15. Let $(X, D)$ be a pair, and $\pi: \widetilde{X} \rightarrow X$ a log resolution $(X, D)$. If $D^{\prime} \subseteq D$ is an effective sub- $\mathbb{Q}$-divisor, then $\pi$ is a log resolution of $\left(X, D^{\prime}\right)$.

Proof. Properties (2.12.1) and (2.12.2) being clear, it remains to show that $\pi^{-1}\left(\operatorname{supp} D^{\prime}\right) \cup$ $\operatorname{Exc}(\pi)$ is a divisor with simple normal crossings. Since every subdivisor of an snc divisor is again an snc divisor, it suffices to show that the set $\pi^{-1}\left(\operatorname{supp} D^{\prime}\right) \cup \operatorname{Exc}(\pi)$ is of pure codimension one. Accordingly, there is nothing to show if either $\operatorname{supp} D^{\prime}=\operatorname{supp} D$, or if $\operatorname{supp} D^{\prime}=\emptyset$. We may thus assume without loss of generality that $\operatorname{supp} D^{\prime} \neq \emptyset$, and that $\operatorname{supp} D^{\prime} \varsubsetneqq \operatorname{supp} D$.

We decompose the preimage of $\operatorname{supp} D^{\prime}$ into a divisorial and a small part,

$$
\pi^{-1}\left(\operatorname{supp} D^{\prime}\right)=\widetilde{D}_{\text {div }}^{\prime} \cup \widetilde{D}_{\text {small }}^{\prime}
$$

where $\widetilde{D}_{\text {div }}^{\prime}$ has pure codimension one, and $\operatorname{codim}_{\widetilde{X}} \widetilde{D}_{\text {small }}^{\prime}>1$. Since supp $D^{\prime}$ is of pure codimension one, it is clear that $\pi$ cannot be isomorphic at general points of $\widetilde{D}_{\text {small }}^{\prime}$, so that $\widetilde{D}_{\text {small }}^{\prime} \subseteq \operatorname{Exc}(\pi)$. It follows that

$$
\pi^{-1}\left(\operatorname{supp} D^{\prime}\right) \cup \operatorname{Exc}(\pi)=\widetilde{D}_{\text {div }} \cup \operatorname{Exc}(\pi) .
$$


Equation 2.15.1 immediately shows that $\pi^{-1}\left(\operatorname{supp} D^{\prime}\right) \cup \operatorname{Exc}(\pi)$ has pure codimension 1 , as claimed. This completes the proof.

2.D. Reflexive sheaves and their tensor operations. The main theme of this paper being reflexive sheaves of differentials on singular spaces, we constantly need to discuss sheaves that are not necessarily locally free. For this, we frequently use square brackets to indicate taking the reflexive hull.

Notation 2.16 (Reflexive tensor operations). Let $X$ be a normal variety, $D$ a reduced Weil divisor, and $\mathscr{A}$ a coherent sheaf of $\mathscr{O}_{X}$-modules. For $n \in \mathbb{N}$, set $\mathscr{A}^{[n]}:=\left(\mathscr{A}^{\otimes n}\right)^{* *}$ and if $\pi: X^{\prime} \rightarrow X$ is a morphism of normal varieties, set $\pi^{[*]}(\mathscr{A}):=\left(\pi^{*} \mathscr{A}\right)^{* *}$. In a similar vein, let $\Omega_{X}^{[p]}:=\left(\Omega_{X}^{p}\right)^{* *}$ and $\Omega_{X}^{[p]}(\log D):=\left(\Omega_{X}^{p}(\log D)\right)^{* *}$ For the definition of $\Omega_{X}^{p}$ and $\Omega_{X}^{p}(\log D)$ see $2 . \mathrm{A}$

Observe that if $(X, D)$ is a pair and $\iota: U=(X, D)_{\text {reg }} \hookrightarrow X$ is the embedding of the regular part of $(X, D)$ in to $X$, then $\Omega_{X}^{[p]}(\log D) \simeq \iota_{*}\left(\Omega_{U}^{p}\left(\left.\log D\right|_{U}\right)\right)$.

Notation 2.17 (Reflexive differential forms). A section in $\Omega_{X}^{[p]}$ or $\Omega_{X}^{[p]}(\log D)$ will be called a reflexive form or a reflexive logarithmic form, respectively.

Generalising the vanishing theorem of Bogomolov-Sommese to singular spaces, we need to discuss the Kodaira-Iitaka dimension of reflexive sheaves. Since this is perhaps not quite standard, we recall the definition here.

Definition 2.18 (Kodaira-Iitaka dimension of a sheaf). Let $X$ be a normal projective variety and $\mathscr{A}$ a reflexive sheaf of rank one on $Z$. If $h^{0}\left(X, \mathscr{A}^{[n]}\right)=0$ for all $n \in \mathbb{N}$, then we say that $\mathscr{A}$ has Kodaira-Iitaka dimension $\kappa(\mathscr{A}):=-\infty$. Otherwise, set

$$
M:=\left\{n \in \mathbb{N} \mid h^{0}\left(X, \mathscr{A}^{[n]}\right)>0\right\},
$$

recall that the restriction of $\mathscr{A}$ to the smooth locus of $X$ is locally free and consider the natural rational mapping

$$
\phi_{n}: X \rightarrow \mathbb{P}\left(H^{0}\left(X, \mathscr{A}^{[n]}\right)^{*}\right) \quad \text { for each } n \in M .
$$

The Kodaira-Iitaka dimension of $\mathscr{A}$ is then defined as

$$
\kappa(\mathscr{A}):=\max _{n \in M}\left(\operatorname{dim} \overline{\phi_{n}(X)}\right) .
$$

Definition 2.19. Let $X$ be a normal algebraic variety. A reflexive sheaf $\mathscr{F}$ of rank one is called $\mathbb{Q}$-Cartier if there exists an $m \in \mathbb{N}^{>0}$ such that $\mathscr{F}[m]$ is locally free.

Remark 2.20. In the setup of Definition 2.19, there exists a reduced Weil divisor $D$ on $X$ such that $\mathscr{F}=\mathscr{O}_{X}(D)$, see for example [Rei80, Appendix to $\left.\S 1\right]$. Then, $\mathscr{F}$ is $\mathbb{Q}$-Cartier if and only if there exists an $m \in \mathbb{N}^{>0}$ such that $\mathscr{O}_{X}(m D)$ is locally free.

2.E. Cutting down. An important technical property of canonical, terminal, klt, dlt and lc singularities is their stability under general hyperplane sections. This is particularly useful in inductive proofs, as we will see, e.g., in Section 9 . We gather the relevant facts here for later reference.

Notation 2.21. For a line bundle $\mathscr{L} \in \operatorname{Pic} X$, the associated linear system of effective Cartier divisors will be denoted by $|\mathscr{L}|$.

Lemma 2.22 (Cutting down pairs I). Let $(X, D)$ be a pair, $\operatorname{dim}(X) \geq 2$, and let $H \in|\mathscr{L}|$ be a general element of an ample basepoint-free linear system corresponding to $\mathscr{L} \in$ Pic $X$. Consider the cycle-theoretic intersection $D_{H}:=D \cap H$. Then the following holds. 
(2.22.1) The divisor $H$ is irreducible and normal.

(2.22.2) If $D=\sum a_{i} D_{i}$ is the decomposition of $D$ into irreducible components, then the intersections $D_{i} \cap H$ are distinct, irreducible and reduced divisors in $H$, and $D_{H}=\sum a_{i}\left(D_{i} \cap H\right)$.

(2.22.3) The tuple $\left(H, D_{H}\right)$ is a pair in the sense of Definition 2.2 and rounding-down $D$ commutes with restriction to $H$, i.e., $\operatorname{supp}\left(\left\lfloor D_{H}\right\rfloor\right)=H \cap \operatorname{supp}(\lfloor D\rfloor)$.

(2.22.4) If $H$ is smooth, then $X$ is smooth along $H$.

(2.22.5) If $\left(H, D_{H}\right)$ is snc, then $(X, D)$ is snc along $H$.

Proof. Assertion (2.22]1] is a known generalisation of Seidenberg's Theorem, see [BS95, Thm. 1.7.1] and [Sei50, Thm. 1]. Assertion (2.22]2] is a well-known consequence of Bertini's theorem, 2.22]3) follows from 2.2211) and 2.2222). Statements (2.22/4)(2.225) are consequences of the fact that a space is smooth along a Cartier divisor if the divisor itself is smooth.

Lemma 2.23 (Cutting down strong $\log$ resolutions). Let $(X, D)$ be a pair, $\operatorname{dim} X \geq 2$, and let $\pi: \widetilde{X} \rightarrow X$ a strong log resolution (resp. a log resolution). Let $H \in|\mathscr{L}|$ be a general element of an ample basepoint-free linear system on $X$ corresponding to $\mathscr{L} \in \operatorname{Pic} X$. Set $\widetilde{H}:=\pi^{-1}(H)$. Then the restricted morphism $\left.\pi\right|_{\widetilde{H}}: \widetilde{H} \rightarrow H$ is a strong log resolution (resp. a log resolution) of the pair $(H, D \cap H)$, with exceptional set $\operatorname{Exc}\left(\left.\pi\right|_{\widetilde{H}}\right)=\operatorname{Exc}(\pi) \cap \widetilde{H}$.

Proof. First consider the case when $\pi$ is a log resolution. Zariski's Main Theorem [Har77, V Thm. 5.2] implies that since $X$ is normal, a point $\widetilde{x} \in \tilde{X}$ is contained in the $\pi$-exceptional set $\operatorname{Exc}(\pi)$ if and only if the fibre through $\widetilde{X}$ is positive dimensional. Since $H$ is normal by (2.22]1), the same holds for the restriction $\left.\pi\right|_{\widetilde{H}}$; for all points $\widetilde{x} \in \widetilde{H}$, we have $\widetilde{x} \in$ $\operatorname{Exc}\left(\left.\pi\right|_{\tilde{H}}\right)$ if and only if the $\pi$-fibre through $\widetilde{x}$ is positive dimensional. It follows that

$$
\begin{array}{rlr}
\operatorname{Exc}\left(\left.\pi\right|_{\widetilde{H}}\right) & =\widetilde{H} \cap \operatorname{Exc}(\pi) & \text { and } \\
\left(\left.\pi\right|_{\widetilde{H}}\right)^{-1}(D \cap H) \cup \operatorname{Exc}\left(\left.\pi\right|_{\widetilde{H}}\right) & =\widetilde{H} \cap(\underbrace{\pi^{-1}(D) \cup \operatorname{Exc}(\pi)}_{\text {snc divisor by assumption }}) .
\end{array}
$$

Since $\pi$ has connected fibres the linear systems $|\mathscr{L}|$ and $\left|\pi^{*} \mathscr{L}\right|$ can be canonically identified. In particular, $\widetilde{H}$ is a general element of a basepoint-free linear system, and it follows immediately from Bertini's Theorem that $\widetilde{H}$ is smooth. The equality in 2.23.1) shows that $\operatorname{Exc}\left(\left.\pi\right|_{\widetilde{H}}\right)$ is of pure codimension one in $\widetilde{H}$. The equality in 2.23.2 and Bertini's Theorem then give that the set $\left(\left.\pi\right|_{\widetilde{H}}\right)^{-1}(D \cap H) \cup \operatorname{Exc}\left(\left.\pi\right|_{\widetilde{H}}\right)$ is a divisor with simple normal crossings. It follows that the restricted map $\left.\pi\right|_{\widetilde{H}}$ is a log resolution of the pair $(H, D \cap H)$.

Now assume that $\pi$ is a strong $\log$ resolution of $(X, D)$. We aim to show that then $\left.\pi\right|_{\widetilde{H}}$ is a strong log resolution of the pair $(H, D \cap H)$. To this end, let $x \in H$ be any point where the pair $(H, D \cap H)$ is snc. By (2.225) the pair $(X, D)$ is then snc in a neighbourhood of $x$, and the strong $\log$ resolution $\pi$ is isomorphic near $x$. The equality in (2.23.1) then shows that the restriction $\left.\pi\right|_{\widetilde{H}}$ is likewise isomorphic near $x$ showing that $\left.\pi\right|_{\widetilde{H}}$ is a strong $\log$ resolution indeed.

Lemma 2.24 (Cutting down pairs II). Let $(X, D)$ be a pair and let $H \in|\mathscr{L}|$ be a general element of an ample basepoint-free linear system corresponding to $\mathscr{L} \in \operatorname{Pic} X$. Consider the cycle-theoretic intersection $D_{H}:=D \cap H$. If $(X, D)$ is dlt (resp. canonical, klt, lc), then $\left(H, D_{H}\right)$ is dlt (resp. canonical, klt, lc) as well. 
Proof. To prove Lemma2.24 for dlt pairs, recall Szabó's characterisation of "dlt" [Sza94], [KM98, Thm. 2.44] which asserts that a pair is dlt if and only if there exists a log resolution $\pi: \widetilde{X} \rightarrow X$ where all exceptional divisors have discrepancy greater than -1 . Choose one such resolution and set $\widetilde{H}:=\pi^{-1}(H)$. Lemma 2.23 then asserts that $\pi_{\widetilde{H}}: \widetilde{H} \rightarrow H$ is a strong log resolution of the pair $\left(H, D_{H}\right)$, and it follows from the adjunction formula that the discrepancy of any $\pi_{\widetilde{H}}$-exceptional divisor is likewise greater than -1 . A second application of the characterisation of dlt pairs then yields the claim in case $(X, D)$ is dlt.

For canonical, klt, or lc pairs, Lemma2.24 follows from a computation of discrepancies, [KM98, Lem. 5.17].

2.F. Projection to subvarieties. Let $X$ be a normal variety such that $X_{\text {sing }}$ is irreducible and of dimension 1. One may study the singularities of $X$ near general points of $X_{\text {sing }}$ by looking at a family of sufficiently general hyperplane sections $\left(H_{t}\right)_{t \in T}$, and by studying the singularities of the hyperplanes $H_{t}$. Near the general point of $X_{\text {sing }}$ the $H_{t}$ define a morphism, and it is often notationally convenient to discuss the family $\left(H_{t}\right)_{t \in T}$ as being fibres of that morphism.

This idea is not new. We include the following proposition to fix notation, and to specify a precise framework for later use.

Proposition 2.25 (Projection to a subvariety). Let $X$ be quasi-projective variety and $T \subseteq$ $X$ an irreducible subvariety. Then there exists a Zariski-open subset $X^{\circ} \subseteq X$ such that $T^{\circ}:=T \cap X^{\circ}$ is not empty, and such that there exists a diagram

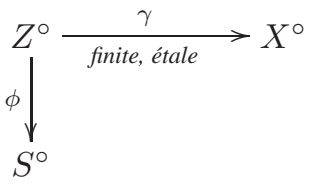

with the property that the restriction of $\phi$ to any connected component of $\widetilde{T}^{\circ}:=\gamma^{-1}\left(T^{\circ}\right)$ is an isomorphism.

Proof. Let $X_{0}^{\circ} \subseteq X$ be an affine open set that intersects $T$ non-trivially. An application of the Noether normalisation theorem, [Sha94, I. Thm. 10], to the affine variety $T_{0}^{\circ}:=$ $T \cap X_{0}^{\circ} \subseteq X_{0}^{\circ}$ yields a projection to an affine space, $\phi_{0}: X_{0}^{\circ} \rightarrow S_{0}^{\circ}$, whose restriction to $T_{0}^{\circ}$ is generically finite. Shrinking $X_{0}^{\circ}$ and $S_{0}^{\circ}$ further, if necessary, we may assume that the restriction $\left.\phi_{0}\right|_{T_{0}^{\circ}}$ is finite and étale, say $n$-to-1. Next, we will construct a commutative diagram of morphisms,

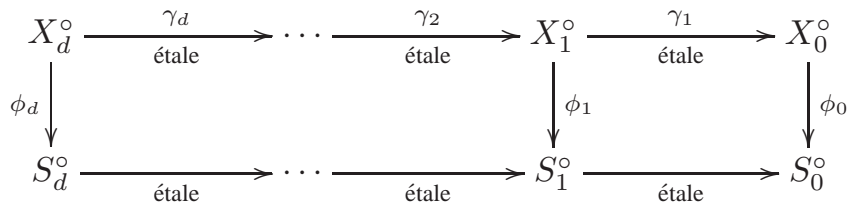

such that

(2.25.2) for any index $k$, the restriction of $\phi_{k}$ to $T_{k}^{\circ}:=\left(\gamma_{1} \circ \cdots \gamma_{k}\right)^{-1}\left(T_{0}^{\circ}\right)$ is étale, and (2.25.3) the restriction of $\phi_{d}$ to any component of $T_{d}^{\circ}$ is isomorphic.

Once the diagram is constructed, the proof is finished by setting $Z^{\circ}:=X_{d}^{\circ}, S^{\circ}:=S_{d}^{\circ}$ and $\phi:=\phi_{d}$.

To construct a diagram as in 2.25.1), we proceed inductively as follows. Assume $\phi_{k}$ : $X_{k}^{\circ} \rightarrow S_{k}^{\circ}$ have already been constructed. If the restriction of $\phi_{k}$ to any component of $T_{k}^{\circ}$ is 
an isomorphism then we stop. Otherwise, let $S_{k+1}^{\circ} \subseteq T_{k}^{\circ}$ be any component where $\left.\phi_{k}\right|_{S_{k+1}^{\circ}}$ is not isomorphic, and set $X_{k+1}^{\circ}:=X_{k}^{\circ} \times S_{k}^{\circ} S_{k+1}^{\circ}$. Since étale morphisms are stable under base change, [Gro71, I Prop. 4.6], it follows that the projection $\gamma_{k+1}: X_{k+1}^{\circ} \rightarrow X_{k}^{\circ}$ and the restriction $\left.\phi_{k+1}\right|_{T_{k+1}^{\circ}}$ are both étale.

We need to show that the inductive process terminates. For that, observe that all restrictions $\left.\phi_{k}\right|_{T_{k}^{\circ}}: T_{k}^{\circ} \rightarrow S_{k}^{\circ}$ are finite, étale and $n$-to- 1 . Additionally, it follows inductively from the fibre product construction that the restriction $\left.\phi_{k}\right|_{T_{k}^{\circ}}$ admits at least $k$ sections. It is then immediate that the process terminates after no more than $n$ steps.

Example 2.26. To illustrate how projections to subvarieties will be used, consider a dlt pair $(X, D)$ whose singular locus $T:=(X, D)_{\operatorname{sing}}$ is irreducible and of codimension $\operatorname{codim}_{X} T=2$. We are often interested in showing properties of the pair $(X, D)$ that can be checked on the étale cover $Z^{\circ}$ constructed in 2.25. Examples for such properties include the following.

(2.26.1) The space $X$ is analytically $\mathbb{Q}$-factorial away from a set of codimension 3 .

(2.26.2) Near the general point of $T$, the space $X$ has only quotient singularities.

(2.26.3) For any strong $\log$ resolution $\pi: \widetilde{X} \rightarrow X$, the sheaf $\pi_{*} \Omega_{\widetilde{X}}^{p}$ is reflexive at the general point of $T$.

Setting $\Delta^{\circ}:=\gamma^{*}(D)$ and considering general fibres

$$
Z_{t}^{\circ}:=\phi^{-1}(t) \quad \text { and } \quad \Delta_{t}^{\circ}:=\Delta^{\circ} \cap Z_{t}^{\circ},
$$

it follows from the Cutting-Down Lemma 2.22 that the fibre pairs $\left(Z_{t}^{\circ}, \Delta_{t}^{\circ}\right)$ are dlt surfaces, where the property in question may often be checked easily. Once it is known that the fibres of $\phi$ have the desired property, it is often possible to prove that the property also holds for the total space $\left(Z^{\circ}, \Delta^{\circ}\right)$ of the family, and hence for $(X, D)$.

\section{EXAMPLES}

In this section we discuss a number of examples that show to what extent the main result of this paper, the Extension Theorem 1.5 is optimal

3.A. Non-log canonical singularities. The next example shows that log canonicity of $(X, D)$ is necessary to obtain any extension result allowing no worse than log poles along the exceptional divisor. This example is discussed in greater detail in [GKK10, Ex. 6.3].

Example 3.1. Let $X$ be the affine cone over a smooth curve $C$ of degree 4 in $\mathbb{P}^{2}$. Observe that $X$ is a normal hyperplane singularity. In particular, $X$ is Gorenstein. Let $\widetilde{X}$ be the total space of the line bundle $\mathscr{O}_{C}(-1)$. Then, the contraction of the zero section $E$ of $\tilde{X}$ yields a strong $\log$ resolution $\pi: \widetilde{X} \rightarrow X$. An elementary computation shows that the discrepancy of $E$ with respect to $X$ is equal to -2 cf. [Rei87, p. 351, Ex. (1)]. Hence, $X$ has worse than $\log$ canonical singularities. If $\tau$ is a local generator of the locally free sheaf $\Omega_{X}^{[2]}$ near the vertex $P \in X$, the discrepancy computation implies that $\tau$ acquires poles of order 2 when pulled back to $\widetilde{X}$. By abusing notation we denote the rational form obtained on $\widetilde{X}$ by $\pi^{*} \tau$.

Next, let $\xi$ be the vector field induced by the natural $\mathbb{C}^{*}$-action on $\widetilde{X}$ coming from the cone structure. By contracting $\pi^{*} \tau$ by $\xi$ we obtain a regular 1-form on $\widetilde{X} \backslash E$ that does not extend to an element of $H^{0}\left(\widetilde{X}, \Omega_{\widetilde{X}}^{1}(\log E)\right)$.

Hence, in the non-log canonical case there is in general no extension result for differential forms, not even for special values of $p$. 
3.B. Non-klt locus and discrepancies. It follows from the definition of discrepancy that for a given reflexive logarithmic $n$-form $\sigma$ on a reduced pair $(X, D)$ of dimension $n$ with $\log$ canonical singularities, the pull-back $\pi^{*} \sigma$ acquires additional poles only along those exceptional divisors $E_{i}$ with discrepancy $a_{i}=-1$, see [GKK10, Sect. 5]. It hence extends without poles even over those divisors $E_{i}$ with discrepancy $a_{i}>-1$ that map to the nonklt locus of $(X, D)$. In the setup of Theorem 1.5 , it is therefore natural to ask whether it is necessary to include the full-preimage of the non-klt locus in $\widetilde{D}$ in order to obtain an extension result or if it suffices to include the non-klt places, that is, those divisor with discrepancy -1 . The next example shows that this does not work in general for extending $p$-forms, when $p<n$.

Example 3.2. Let $X=\left\{u w-v^{2}\right\} \subset \mathbb{C}_{u, v, w}^{3}$ be the quadric cone, and let $D=\{v=$ $0\} \cap X$ be the union of two rays through the vertex. The pair $(X, D)$ is $\log$ canonical. Let $\tilde{X} \subset \mathrm{Bl}_{(0,0,0)}\left(\mathbb{C}^{3}\right) \subset \mathbb{C}_{u, v, w}^{3} \times \mathbb{P}_{\left[y_{1}: y_{2}: y_{3}\right]}^{2}$ be the strict transform of $X$ in the blowup of $\mathbb{C}^{3}$ at $(0,0,0)$ and $\pi: \widetilde{X} \rightarrow X$ the corresponding resolution. The intersection $U$ of $\widetilde{X}$ with $\left\{y_{1} \neq 0\right\}$ is isomorphic to $\mathbb{C}^{2}$ and choosing coordinates $x, z$ on this $\mathbb{C}^{2}$, the blow-up is given by $\varphi:(x, z) \mapsto\left(z, x z, x^{2} z\right)$. In these coordinates the exceptional divisor $E$ is defined by the equation $\{z=0\}$. The form $d \log v:=\frac{1}{v} d v$ defines an element in $H^{0}\left(X, \Omega_{X}^{[1]}(\log D)\right)$. Pulling back we obtain

$$
\varphi^{*}(d \log v)=d \log x+d \log z .
$$

which has log-poles along the exceptional divisor. If $f: \widetilde{X}^{\prime} \rightarrow \widetilde{X}$ is the blow up at a point $p \in E \backslash \pi_{*}^{-1}(D)$, we obtain a further resolution $\pi^{\prime}=\pi \circ f$ of $X$. This resolution has an additional exceptional divisor $E^{\prime} \subset \widetilde{X}^{\prime}$ with discrepancy 0 . Note however that the pull-back of $d \log v$ via $\pi^{\prime}$ has logarithmic poles along $E^{\prime}$. To be explicit we compute on $f^{-1}(U)$ : we have

$$
f^{*} \varphi^{*}(d \log v)=d \log \left(f^{*} x\right)+d \log \left(f^{*} z\right),
$$

and we note that $f^{*} z$ vanishes along $E^{\prime}$ since we have blown up a point in $E=\{z=0\}$.

3.C. Other tensor powers. The statement of Theorem 1.5 does not hold for arbitrary reflexive tensor powers of $\Omega_{X}^{1}$. We refer to [GKK10, Ex. 3.1.3] for an example where the analogue of the Extension Theorem 1.5 fails for $\operatorname{Sym}^{[2]} \Omega_{X}^{1}$, even when $X$ is canonical.

\section{PART II. APPLICATIONS OF THE EXTENSION THEOREM}

\section{PULL-BACK MORPHISMS FOR REFLEXIVE DIFFERENTIALS}

Kähler differentials are characterised by a number of universal properties, one of the most important being the existence of a pull-back map: if $\gamma: Z \rightarrow X$ is any morphism of algebraic varieties and if $p \in \mathbb{N}$, then there exists a canonically defined sheaf morphism

$$
d \gamma: \gamma^{*} \Omega_{X}^{p} \rightarrow \Omega_{Z}^{p}
$$

The following example illustrates that for sheaves of reflexive differentials on normal spaces, a pull-back map does not exist in general.

Example 4.2 (Pull-back morphism for dualising sheaves). Let $X$ be a normal Gorenstein variety of dimension $n$, and let $\gamma: Z \rightarrow X$ be any resolution of singularities. Observing that the sheaf of reflexive $n$-forms is precisely the dualising sheaf, $\Omega_{X}^{[n]} \simeq \omega_{X}$, it follows directly from the definition of canonical singularities that $X$ has canonical singularities if and only if a pull-back morphism $d \gamma: \gamma^{*} \Omega_{X}^{[n]} \rightarrow \Omega_{Z}^{n}$ exists. 


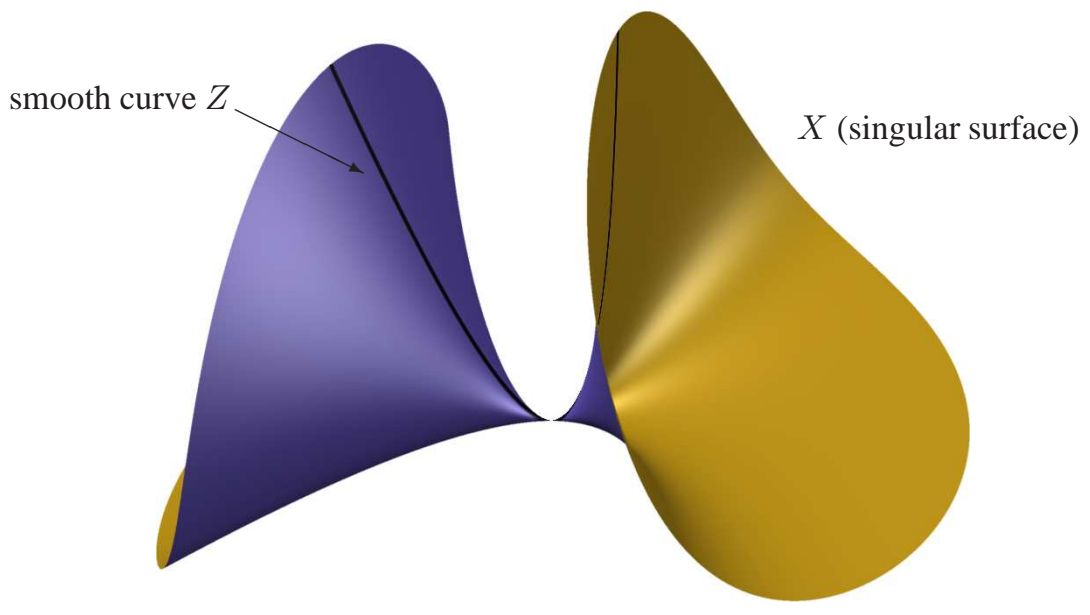

FIGURE 1. A special case of the Pull-back theorem 4.3.

An important consequence of the Extension Theorem 1.5] is the existence of a pull-back map for reflexive differentials of arbitrary degree, whenever $\gamma: Z \rightarrow X$ is a morphism where the target is klt. The pull-back map exists also in the logarithmic setup and -in a slightly generalised form - in cases where the target is only lc.

Theorem 4.3 (Pull-back map for reflexive differentials on lc pairs). Let $(X, D)$ be an lc pair, and let $\gamma: Z \rightarrow X$ be a morphism from a normal variety $Z$ such that the image of $Z$ is not contained in the reduced boundary or in the singular locus, i.e.,

$$
\gamma(Z) \nsubseteq(X, D)_{\operatorname{sing}} \cup \operatorname{supp}\lfloor D\rfloor .
$$

If $1 \leq p \leq \operatorname{dim} X$ is any index and

$$
\Delta:=\text { largest reduced Weil divisor contained in } \gamma^{-1} \text { (non-klt locus), }
$$

then there exists a sheaf morphism,

$$
d \gamma: \gamma^{*} \Omega_{X}^{[p]}(\log \lfloor D\rfloor) \rightarrow \Omega_{Z}^{[p]}(\log \Delta),
$$

that agrees with the usual pull-back morphism (4.1) of Kähler differentials at all points $p \in Z$ where $\gamma(p) \notin(X, D)_{\text {sing }} \cup \operatorname{supp}\lfloor D\rfloor$.

Before proving Theorem 4.3 below, we illustrate the statement with one example and add a remark concerning possible generalisations.

Example 4.4 (Restriction as a special case of Theorem 4.3). For a special case of Theorem 4.3, consider the case sketched in Figure 1, where $(X, \emptyset)$ is klt and $Z \subset X$ is a smooth subvariety that intersects $X_{\text {reg }}$ non-trivially with inclusion map $\gamma: Z \rightarrow X$. Under these assumptions, Theorem 4.3 asserts that any reflexive differential form $\sigma \in H^{0}\left(X, \Omega_{X}^{[p]}\right)$ restricts to a regular form on $Z$.

Remark 4.5 (Pull-back map when the image is contained in the boundary). In the setup of Theorem 4.3, if we assume additionally that the pair $(X, D)$ is dlt, then one may use the residue sequence (11.711 of Theorem 11.7 to define a pull-back map even in a setting where the image of $\gamma$ is contained in the boundary $\lfloor D\rfloor$. Details will be published in a forthcoming paper. 
The proof of Theorem 4.3 uses the following notation.

Notation 4.6. Let $(X, D)$ and $(Z, \Delta)$ be two pairs, and $\gamma: Z \rightarrow X$ a morphism such that $\gamma(Z) \nsubseteq(X, D)_{\text {sing }} \cup \operatorname{supp}\lfloor D\rfloor$. If $\sigma$ is a rational section in $\Omega_{X}^{[p]}(\log \lfloor D\rfloor)$, then one may use the standard pull-back map for Kähler differentials to pull $\sigma$ back to a rational section of $\Omega_{Z}^{[p]}(\log \lfloor\Delta\rfloor)$, which we denote by $\gamma^{*}(\sigma)$.

Proof of Theorem 4.3 Notice that to prove Theorem 4.3 it suffices to show that for every open subset $V \subseteq X$ the following holds:

$$
\gamma^{*}(\sigma) \in H^{0}\left(\gamma^{-1}(V), \Omega_{Z}^{[p]}(\log \Delta)\right) \text { for all } \sigma \in H^{0}\left(V, \Omega_{X}^{[p]}(\log \lfloor D\rfloor)\right) .
$$

Indeed, for every point $p \in Z$ and every germ $s \in\left(\gamma^{*} \Omega_{X}^{[p]}(\log \lfloor D\rfloor)\right)_{p}$ there exists an open neighbourhood $U$ of $p$ in $Z$, an open neighbourhood $V$ of $\gamma(p)$ in $X$ such that $\gamma(U) \subseteq$ $V$, and such that $s$ is represented by a sum $\sum g_{j} \cdot \gamma^{*} \sigma_{j}$, where $g_{j} \in \mathscr{O}_{Z}(U)$ and $\sigma_{j} \in$ $H^{0}\left(V, \Omega_{X}^{[p]}(\log \lfloor D\rfloor)\right)$.

To prove 4.6.1), let $\sigma \in H^{0}\left(V, \Omega_{X}^{[p]}(\log \lfloor D\rfloor)\right)$ be any reflexive form. To simplify notation, we may assume without loss of generality that $V=X$ and $\gamma^{-1}(V)=Z$. Let $\pi: \widetilde{X} \rightarrow X$ be any strong resolution of the pair $(X, D)$ and consider the following commutative diagram of varieties,

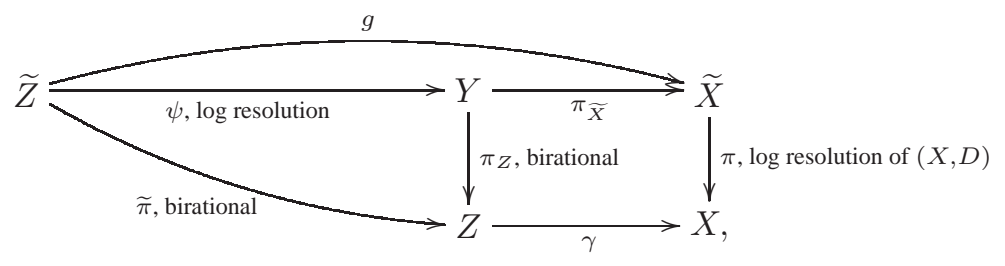

where $Y$ is the normalisation of the unique component of $Z \times_{X} \widetilde{X}$ that dominates $Z$, and where $\psi$ is a $\log$ resolution of the pair $\left(Y,\left(\pi \circ \pi_{\tilde{X}}\right)^{*} D\right)$. Furthermore, set

$$
\begin{aligned}
\widetilde{D} & :=\text { largest reduced divisor in } \operatorname{supp} \pi^{-1}(\text { non-klt locus of }(X, D)), \\
\widetilde{\Delta} & :=\text { largest reduced divisor in } \operatorname{supp}(\pi \circ g)^{-1}(\text { non-klt locus of }(X, D)) .
\end{aligned}
$$

By definition, we immediately obtain two relations 1 involving cycle-theoretic pull-back and push-forward,

$$
\begin{aligned}
& \operatorname{supp} g^{*} \widetilde{D} \subseteq \operatorname{supp} \widetilde{\Delta}, \\
& \operatorname{supp} \widetilde{\pi}_{*} \widetilde{\Delta}=\operatorname{supp} \Delta .
\end{aligned}
$$

It is then clear from 4.6.3) that 4.6.1) holds once we show that

$$
\widetilde{\pi}^{*}\left(\gamma^{*}(\sigma)\right)=g^{*}\left(\pi^{*}(\sigma)\right) \in H^{0}\left(\widetilde{Z}, \Omega_{\widetilde{Z}}^{[p]}(\log \widetilde{\Delta})\right) .
$$

The Extension Theorem 1.5 states that the pull-back $\pi^{*}(\sigma)$ is a regular logarithmic form in $H^{0}\left(\widetilde{X}, \Omega_{\widetilde{X}}^{p}(\log \widetilde{D})\right)$, for all reflexive forms $\sigma$. Using (4.6.2) and the standard pull-back map for logarithmic forms on snc pairs to pull back $\pi^{*} \sigma$ via the map $g$, the desired inclusion in (4.6.4 follows. This completes the proof.

\footnotetext{
${ }^{1}$ Note that the inclusion in 4.6 .2 might be strict. This can happen when $\pi^{-1}$ (non-klt locus of $(X, D)$ ) contains components of high codimension whose preimages under $g$ become divisors.
} 


\section{REFLEXIVE DIFFERENTIALS ON RATIONALLY CHAIN CONNECTED SPACES}

Rationally chain connected manifolds are rationally connected, and do not carry differential forms. Building on work of Hacon and McKernan, [HM07], we show that the same holds for reflexive forms on klt pairs.

Theorem 5.1 (Reflexive differentials on rationally chain connected spaces). Let $(X, D)$ be a klt pair. If $X$ is rationally chain connected, then $X$ is rationally connected, and $H^{0}\left(X, \Omega_{X}^{[p]}\right)=0$ for all $p \in \mathbb{N}, 1 \leq p \leq \operatorname{dim} X$.

Proof. Choose a strong $\log$ resolution $\pi: \widetilde{X} \rightarrow X$ of the pair $(X, D)$. Since klt pairs are also dlt, a theorem of Hacon-McKernan, [HM07, Cor. 1.5(2)], applies to show that $X$ and $\widetilde{X}$ are both rationally connected. In particular, it follows that $H^{0}\left(\widetilde{X}, \Omega_{\widetilde{X}}^{p}\right)=0$ for all $p>0$ by [Kol96, IV. Cor. 3.8].

Since $(X, D)$ is klt, Theorem 4.3 asserts that there exists a pull-back morphism $d \pi$ : $\pi^{*} \Omega_{X}^{[p]} \rightarrow \Omega_{\widetilde{X}}^{p}$. As $\pi$ is birational, $d \pi$ is generically injective and since $\Omega_{X}^{[p]}$ is torsion-free, this means that the induced morphism on the level of sections is injective:

$$
\pi^{*}: H^{0}\left(X, \Omega_{X}^{[p]}\right) \rightarrow H^{0}\left(\widetilde{X}, \Omega_{\widetilde{X}}^{p}\right)=0 .
$$

The claim then follows.

In this section, Theorem 5.1 is presented as a consequence of the Extension Theorem 1.5. As a matter of fact, the proof of the Extension Theorem 1.5. which we give in Part VI of the paper, involves a proof of Theorem 5.1 as part of the induction process. This explains why the statement of Theorem 5.1 appears essentially unchanged as Proposition 19.4 in Part V1] where the Extension Theorem 1.5 is proven.

In order to avoid confusion about the logic of this paper, we have chosen to present an independent statement and an independent proof here.

\section{THE LIPMAN-ZARISKI CONJECTURE FOR KLT SPACES}

The Lipman-Zariski Conjecture asserts that a variety $X$ with a locally free tangent sheaf $\mathscr{T}_{X}$ is necessarily smooth, [Lip65]. The conjecture has been shown in special cases; for hypersurfaces or homogeneous complete intersections [Hoc75, SS72], for isolated singularities in higher-dimensional varieties [SvS85, Sect. 1.6], and more generally, for varieties whose singular locus has codimension at least 3 [Fle88]. In this section we use the Extension Theorem 1.5 to prove the Lipman-Zariski Conjecture for klt spaces. Notice that klt spaces in general have singularities in codimension 2. The proof follows an argument that goes back at least as far as [SvS85]. It uses the notion of logarithmic tangent sheaf, which we quickly recall: if $Z$ is a smooth algebraic variety and $\Delta$ is an snc divisor on $Z$, then the logarithmic tangent sheaf $\mathscr{T}_{Z}(-\log \Delta)$ is defined to be the dual of $\Omega_{Z}^{1}(\log \Delta)$. A local computation shows that $\mathscr{T}_{Z}(-\log \Delta)$ can be identified with the subsheaf of $\mathscr{T}_{Z}$ containing those vector fields that are tangent to $\Delta$ at smooth points of $\Delta$.

Theorem 6.1 (Lipman-Zariski Conjecture for klt spaces). Let $X$ be a klt space such that the tangent sheaf $\mathscr{T}_{X}$ is locally free. Then $X$ is smooth.

Proof. We argue by contradiction and assume that $X$ is not smooth. Recall that there exists a uniquely defined strong log resolution $\pi: \widetilde{X} \rightarrow X$ of the pair $(X, \emptyset)$, called the "functorial" resolution, that is universal in the sense that it commutes with smooth morphisms, see [Kol07, Thms. 3.35 and 3.45]. The $\pi$-exceptional set $E$ will then be a non-empty divisor in $\widetilde{X}$, with snc support. 
Next, let $\theta_{1}, \ldots, \theta_{n}$ be sections in $\mathscr{T}_{X}$ that freely generate $\mathscr{T}_{X}$ in a neighbourhood $U$ of a given point $x \in X$. For simplicity of notation, we assume in the following that $U=X$. Given that $\pi$ is the functorial resolution, and that the singular set $X_{\text {sing }}$ is invariant under any automorphism, it follows from [GKK10, Cor. 4.7] that we may lift each $\theta_{j}$ to a logarithmic vector field on $\widetilde{X}$,

$$
\widetilde{\theta}_{j} \in H^{0}\left(\widetilde{X}, \mathscr{T}_{\widetilde{X}}(-\log E)\right) \subseteq H^{0}\left(\widetilde{X}, \mathscr{T}_{\widetilde{X}}\right) .
$$

Notice that away from $E$, the vector fields $\widetilde{\theta}_{j}$ are linearly independent. Choosing the dual basis, we will therefore obtain a set of differential forms

$$
\omega_{1}, \ldots, \omega_{n} \in H^{0}\left(\widetilde{X} \backslash E, \Omega_{\widetilde{X}}^{1}\right) \quad \text { such that } \forall i, j: \omega_{i}\left(\left.\widetilde{\theta}_{j}\right|_{\widetilde{X} \backslash E}\right)=\delta_{i j} \cdot \mathbf{1}_{\widetilde{X} \backslash E},
$$

where $\mathbf{1}_{\widetilde{X} \backslash E}$ is the constant function on $\widetilde{X} \backslash E$ with value 1. By the Extension Theorem 1.5 and Remark 1.5.2, the $\omega_{i}$ extend to differential forms that are defined on all of $\widetilde{X}$,

$$
\widetilde{\omega}_{1}, \ldots, \widetilde{\omega}_{n} \in H^{0}\left(\widetilde{X}, \Omega_{\widetilde{X}}^{1}\right) \text { such that } \forall i, j: \widetilde{\omega}_{i}\left(\widetilde{\theta}_{j}\right)=\delta_{i j} \cdot \mathbf{1}_{\widetilde{X}} .
$$

Now, if we evaluate the vector fields $\widetilde{\theta}_{j} \in H^{0}\left(\widetilde{X}, \mathscr{T}_{\widetilde{X}}\right)$ at any smooth point $p$ of $E$, the inclusion in 6.1.1) shows that the tangent vectors obtained,

$$
\theta_{1}(p), \ldots, \theta_{n}(p) \in \mathscr{T}_{\widetilde{X}} \otimes \kappa(p)
$$

actually lie in $\mathscr{T}_{E} \otimes \kappa(p)$. In particular, the tangent vectors $\theta_{i}(p)$ are linearly dependent. This contradicts (6.1.2) and completes the proof.

\section{BOGOMOLOV-SOMMESE TYPE RESULTS ON LOG CANONICAL SPACES}

7.A. Introduction and statement of the result. In this section, we use the Extension Theorem 1.5 to generalise the Bogomolov-Sommese vanishing theorem to the log canonical setting and to Campana's "geometric orbifolds". In its standard version, [EV92, Cor. 6.9], the theorem limits positivity of invertible sheaves of differentials, asserting that for any reduced snc pair $(X, D)$, any invertible sheaf of $p$-forms has Kodaira-Iitaka dimension no more than $p$, i.e.,

$$
\forall \text { invertible } \mathscr{A} \subseteq \Omega_{X}^{p}(\log D): \kappa(\mathscr{A}) \leq p,
$$

Theorem 7.2 the main result of this section, asserts that the inequality (7.1) also holds in the log canonical setting, for arbitrary $\mathbb{Q}$-Cartier sheaves of rank one (in the sense of Definition 2.19.

For three-dimensional reduced pairs $(X, D)$ this was proven in [GKK10, Thm. 1.4]. This three-dimensional case was an important ingredient in the generalisation of Shafarevich hyperbolicity to families over two- and three-dimensional base manifolds, KK07, KK10a]. There is hope that Theorem 7.2 will allow to generalise Shafarevich hyperbolicity to families over base manifolds of arbitrary dimension.

Theorem 7.2 (Bogomolov-Sommese vanishing for lc pairs). Let $(X, D)$ be an lc pair, where $X$ is projective. If $\mathscr{A} \subseteq \Omega_{X}^{[p]}(\log \lfloor D\rfloor)$ is a $\mathbb{Q}$-Cartier reflexive subsheaf of rank one, then $\kappa(\mathscr{A}) \leq p$.

Remark 7.2.1. The number $\kappa(\mathscr{A})$ appearing in the statement of Theorem 7.2 is the generalised Kodaira-Iitaka dimension introduced in Definition 2.18 on page 8 .

A proof of Theorem 7.2 is given in Section $7 . \mathrm{C}$ on the next page. 
7.B. Bogomolov-Sommese vanishing in the orbifold setting. In [Cam04], Campana introduced the category of "geometric orbifolds". These are pairs $(X, D)$ where all coefficients of the boundary divisor $D$ are of special form. Geometric orbifolds can in many ways be seen as interpolating between the compact and the logarithmic setup. As the word "geometric orbifold" is perhaps not universally accepted in this context, we prefer to call $(X, D)$ a "C-pair" in this paper. A brief overview and precise definitions for all notions that are relevant to our discussion are found in [JK09a, Part I].

Essentially all notions used in the compact or logarithmic setup can be generalised to $\mathcal{C}$-pairs. Examples include the following.

- Given $p, q \in \mathbb{N}$, there exist reflexive sheaves of $\mathcal{C}$-differentials $\operatorname{Sym}_{\mathcal{C}}^{[q]} \Omega_{X}^{p}(\log D)$, [JK09a, Sect. 3.5], with inclusions

$$
\operatorname{Sym}^{[q]} \Omega_{X}^{[p]}(\log \lfloor D\rfloor) \subseteq \operatorname{Sym}_{\mathcal{C}}^{[q]} \Omega_{X}^{p}(\log D) \subseteq \operatorname{Sym}^{[q]} \Omega_{X}^{[p]}(\log \lceil D\rceil) .
$$

In case $q=1$ one has the equality $\operatorname{Sym}_{\mathcal{C}}^{[1]} \Omega_{X}^{p}(\log D)=\Omega_{X}^{[p]}(\log \lfloor D\rfloor)$.

- Given a reflexive subsheaf $\mathscr{A} \subseteq \operatorname{Sym}_{\mathcal{C}}^{[1]} \Omega_{X}^{p}(\log D)$ of rank one, there exists a notion of a $\mathcal{C}$-Kodaira dimension, denoted by $\kappa_{\mathcal{C}}(\mathscr{A})$ that takes fractional parts of $D$ into account, [JK09a, Def. 4.3]. In general, one has $\kappa_{\mathcal{C}}(\mathscr{A}) \geq \kappa(\mathscr{A})$.

Sheaves of $\mathcal{C}$-differentials seem particularly suitable for the discussion of positivity on moduli spaces, cf. [JK09b] In this context, the following strengthening of Theorem 7.2 promises to be of great importance.

Theorem 7.3 (Bogomolov-Sommese vanishing for lc $\mathcal{C}$-pairs). Let $(X, D)$ be a $\mathcal{C}$-pair. Assume that $X$ is projective and $\mathbb{Q}$-factorial, that $\operatorname{dim} X \leq 3$, and that the pair $(X, D)$ is lc. If $1 \leq p \leq \operatorname{dim} X$ is any number and if $\mathscr{A} \subseteq \operatorname{Sym}_{\mathcal{C}}^{[1]} \Omega_{X}^{p}(\log D)$ is a reflexive sheaf of rank one, then $\kappa_{\mathcal{C}}(\mathscr{A}) \leq p$.

Remark 7.3.1. The important point in Theorem 7.3 is the use of the $\mathcal{C}$-Kodaira dimension $\kappa_{\mathcal{C}}(\mathscr{A})$ instead of the usual Kodaira dimension of $\mathscr{A}$.

Proof of Theorem 7.3 Using the Bogomolov-Sommese vanishing theorem for lc pairs, Theorem 7.2 instead of the weaker version [GKK10, Thm. 1.4], the proof from [JK09a, Sect. 7] applies verbatim.

7.C. Proof of Theorem 7.2. We argue by contradiction and assume that there exists a reflexive subsheaf $\mathscr{A} \subseteq \Omega_{X}^{[p]}(\log \lfloor D\rfloor)$ with Kodaira-Iitaka dimension $\kappa(\mathscr{A})>p$. Let $\pi: \widetilde{X} \rightarrow X$ be a strong $\log$ resolution of the pair $(X, D)$. We consider the following reduced snc divisors on $\widetilde{X}$,

$$
\begin{array}{ll}
E & :=\pi \text {-exceptional set, } \\
E^{\prime} & :=\operatorname{supp}\left(\pi_{*}^{-1} D+E\right) \\
\widetilde{D} & :=\text { largest reduced divisor in } \pi^{-1}(\text { non-klt locus of }(X, D))
\end{array}
$$

Since $\widetilde{D} \subseteq E^{\prime}$, the Pull-Back Theorem 4.3 for reflexive differentials implies that there exists an embedding $\pi^{[*]} \mathscr{A} \hookrightarrow \Omega_{\widetilde{X}}^{p}\left(\log E^{\prime}\right)$. Let $\mathscr{C} \subseteq \Omega_{\widetilde{X}}^{p}\left(\log E^{\prime}\right)$ be the saturation of the image, which is reflexive by [OSS80, Lem. 1.1.16 on p. 158], and in fact invertible by [OSS80, Lem. 1.1.15 on p. 154]. Further observe that for any $k \in \mathbb{N}$, the subsheaf $\mathscr{C}^{\otimes k} \subseteq \operatorname{Sym}^{k} \Omega_{\widetilde{X}}^{p}\left(\log E^{\prime}\right)$ is likewise saturated. To prove Theorem 7.2 it suffices to show that

$$
\kappa(\mathscr{C}) \geq \kappa(\mathscr{A})>p
$$


which contradicts the standard Bogomolov-Sommese Vanishing Theorem for snc pairs, [EV92, Cor. 6.9].

Choosing a basis of sections. Choose a number $m$ such that $\operatorname{dim} \overline{\phi_{m}(X)}=\kappa(\mathscr{A})=: \kappa$, where $\phi_{m}$ is the rational map used in the definition of Kodaira dimension, Definition 2.18 on page 8 . Let $B:=\left\{\sigma_{1}, \ldots, \sigma_{\kappa}\right\}$ be a a basis of $H^{0}\left(X, \mathscr{A}^{[m]}\right)$. If $\sigma \in B$ is any element, consider the pull-back $\pi^{*}(\sigma)$, which is a rational section in $\mathscr{C}^{\otimes m}$, possibly with poles along the exceptional set $E$. To show (7.3.2), it suffices to prove that $\pi^{*}(\sigma)$ does not have any poles as a section in $\mathscr{C}^{\otimes m}$, i.e., that

$$
\pi^{*}(\sigma) \in H^{0}\left(\tilde{X}, \mathscr{C}^{\otimes m}\right) \quad \forall \sigma \in B .
$$

Since $\mathscr{C}^{\otimes m}$ is saturated in $\operatorname{Sym}^{m} \Omega_{\widetilde{X}}^{p}\left(\log E^{\prime}\right)$, to show 7.3 .3 , it suffices to show that the $\pi^{*}(\sigma)$ do not have any poles as sections in the sheaf of symmetric differentials, i.e., that

$$
\pi^{*}(\sigma) \in H^{0}\left(\widetilde{X}, \operatorname{Sym}^{m} \Omega_{\widetilde{X}}^{p}\left(\log E^{\prime}\right)\right) \quad \forall \sigma \in B
$$

Taking an index-one-cover. The statement of (7.3.4 is local on $X$, hence we may shrink $X$ and assume that a suitable reflexive tensor power of $\mathscr{A}$ is trivial, say $\mathscr{A}^{[r]} \simeq \mathscr{O}_{X}$. Let $\gamma: Z \rightarrow X$ be the associated index-one-cover, cf. [KM98, Def. 2.52], [HK10, Sect. 2.D]. Let $D=\sum_{i} d_{i} D_{i}$ where $D_{i}$ are reduced irreducible divisors and $d_{i} \in \mathbb{Q}^{>0}$. Given any index $i$, let $\Delta_{i}:=\gamma^{-1}\left(D_{i}\right)$ be the reduced irreducible divisor supported on $\gamma^{-1}\left(\operatorname{supp} D_{i}\right)$, and set $\Delta:=\sum_{i} d_{i} \Delta_{i}$. Since $\gamma$ is étale in codimension 1 by construction, it follows that $K_{Z}+\Delta=\gamma^{*}\left(K_{X}+D\right)$ and hence the pair $(Z, \Delta)$ is again lc by [KM98, Prop. 5.20]. Furthermore, the sheaf $\mathscr{B}:=\gamma^{[*]}(\mathscr{A})$ is a locally free subsheaf of $\Omega_{Z}^{[p]}(\log \lfloor\Delta\rfloor)$, with section

$$
\sigma_{Z}:=\gamma^{[*]}(\sigma) \in H^{0}\left(Z, \mathscr{B}^{\otimes m}\right)
$$

A partial resolution of $Z$. Next, consider the commutative diagram

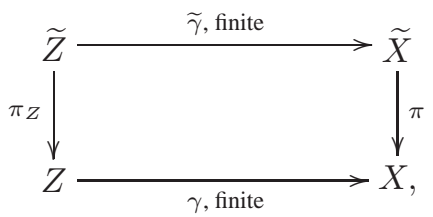

where $\widetilde{Z}$ is the normalisation of the fibre product $Z \times_{X} \widetilde{X}$. We consider the following reduced divisors on $\widetilde{Z}$,

$$
\begin{array}{lll}
E_{Z} & :=\pi_{Z} \text {-exceptional set } & =\operatorname{supp} \widetilde{\gamma}^{*} E, \\
E_{Z}^{\prime} & :=\operatorname{supp}\left((\pi \circ \widetilde{\gamma})_{*}^{-1}(D)+\widetilde{E}\right) & =\operatorname{supp} \widetilde{\gamma}^{*} E^{\prime}, \\
\widetilde{\Delta} & :=\text { largest reduced divisor in } \pi^{-1}(\text { non-klt locus of }(Z, \Delta)) &
\end{array}
$$

The inclusion $\widetilde{\Delta} \subseteq E_{Z}^{\prime}$ and Theorem 4.3 gives an embedding $\pi_{Z}^{*} \mathscr{B} \hookrightarrow \Omega_{\widetilde{Z}}^{[p]}\left(\log E_{Z}^{\prime}\right)$. In fact, since $\mathscr{B}$ is locally free, we also obtain an embedding of tensor powers,

$$
\iota_{m}: \pi_{Z}^{*} \mathscr{B}^{\otimes m} \hookrightarrow \operatorname{Sym}^{[m]} \Omega_{\widetilde{Z}}^{[p]}\left(\log E_{Z}^{\prime}\right) .
$$


Completion of proof. Since the index-one-cover $\gamma$ is étale away from the singularities of $X$, the morphism $\widetilde{\gamma}$ is étale outside of $E \subseteq E^{\prime}$. In particular, the standard pull-back morphism of logarithmic differentials, defined on the smooth locus of $\widetilde{Z}$, gives an isomorphism

$$
\widetilde{\gamma}^{[*]}\left(\operatorname{Sym}^{m} \Omega_{\widetilde{X}}^{p}\left(\log E^{\prime}\right)\right) \simeq \operatorname{Sym}^{[m]} \Omega_{\widetilde{Z}}^{[p]}\left(\log E_{Z}^{\prime}\right) .
$$

This isomorphism implies that in order to prove (7.3.4), it suffices to show that

$$
\widetilde{\gamma}^{[*]}\left(\pi^{*}(\sigma)\right) \in H^{0}\left(\widetilde{Z}, \operatorname{Sym}^{[m]} \Omega_{\widetilde{Z}}^{[p]}\left(\log E_{Z}^{\prime}\right)\right) .
$$

The inclusion in (7.3.5), however, follows when we observe that the rational section $\widetilde{\gamma}^{[*]}\left(\pi^{*}(\sigma)\right)$ of $\operatorname{Sym}^{[m]} \Omega_{\widetilde{Z}}^{[p]}\left(\log E_{Z}^{\prime}\right)$ and the regular section $\iota_{m}\left(\sigma_{Z}\right)=\widetilde{\pi}^{[*]}\left(\sigma_{Z}\right)$ agree on the open set $\widetilde{Z} \backslash \operatorname{supp} E_{Z}$. This finishes the proof Theorem 7.2

\section{PART III. REFLEXIVE FORMS ON DLT PAIRS}

\section{Overview AND MAIN RESUlts of PART III}

8.A. Introduction. Logarithmic Kähler differentials on snc pairs are canonically defined. They are characterised by strong universal properties and appear accordingly in a number of important sequences, filtered complexes and other constructions. First examples include the following:

(8.1.1) the pull-back property of differentials under arbitrary morphisms,

(8.1.2) relative differential sequences for smooth morphisms,

(8.1.3) residue sequences associated with snc pairs, and

(8.1.4) the description of Chern classes as the extension classes of the first residue sequence.

On singular spaces, Kähler differentials enjoy similar universal properties, but the sheaves of Kähler differentials are hardly ever normal, often contain torsion parts and are notoriously hard to deal with. For one example of the problems arising with Kähler differentials, observe that $\Omega_{X}^{p}$ is generally not pure in the sense of [HL97, Def. 1.1.2], so that no Harder-Narasimhan filtration ever exists.

Many of these problems can be overcome by using the sheaves $\Omega_{X}^{[p]}$ of reflexive differentials. For instance, Harder-Narasimhan filtrations exist for $\Omega_{X}^{[p]}$, sheaves of reflexive differentials enjoy good push-forward properties, [KK10a, Lem. 5.2], and reflexive differential can be constructed using Hodge-theoretic methods in a number of settings that are of interest for moduli theory, see for instance [VZ02, Thm. 1.4] and the application in [KK10a, Thm. 5.3].

Reflexive differentials do in general not enjoy the same universal properties as Kähler differentials. However, we have seen in Section 4.3 as one consequence of the Extension Theorem that reflexive differentials do have good pull-back properties if we are working with dlt pairs, and that an analogue of the property (8.1.1) holds. In the present Part of this paper, we would like to make the point that each of the Properties (8.1.2)-(8.1.4) has a very good analogue for reflexive differentials if we are working with dlt pairs. This makes reflexive differential extremely useful in practise. In a sense, it seems fair to say that "reflexive differentials and dlt pairs are made for one another".

8.B. Overview of Part III. We recall the precise statements of the properties (8.1.2)(8.1.4), formulate and prove generalisations to singular spaces in Sections 10-12 below.

Unlike the property (8.1.1), whose generalisation to singular spaces is given in Theorem 4.3 as a corollary of our main result, the results of this section do not depend on the 
Extension Theorem 1.5, but follow from a detailed analysis of the local analytic codimension 2 structure of dlt pairs. We have therefore included a preparatory Section 9 devoted to the discussion of dlt pairs.

\section{THE LOCAL STRUCTURE OF DLT PAIRS IN CODIMENSION 2}

The proofs of the results announced in the previous Section 8 will be given in Sections $10-12$ below. To prepare for the proofs, this section contains a detailed analysis of singularities that appear in the minimal model program. Since we are concerned with reflexive differentials and their restrictions to boundary components, we are mostly interested in structure results that hold in codimension 2.

Although the statements proven in this section are probably known to experts, to the best of our knowledge, proofs of these are not available in the literature. Since our arguments in other parts of the paper crucially depend on the detailed knowledge about the structure of dlt pairs as presented in this section, we have therefore chosen to include proofs of all statements required later, also for the reader's convenience.

9.A. $\mathbb{Q}$-factoriality of dlt pairs in codimension 2. If $(X, D)$ is a dlt surface pair, it is well-understood that $X$ is automatically $\mathbb{Q}$-factorial, [KM98, Prop. 4.11]. This remains true even if $(X, D)$ is only assumed to be numerically dlt and $K_{X}+D$ is not assumed to be $\mathbb{Q}$-Cartier. A higher dimensional dlt pair is not necessarily $\mathbb{Q}$-factorial, but the underlying space of a dlt pair is always $\mathbb{Q}$-factorial in codimension 2 regardless of its dimension.

Proposition 9.1 ( $\mathbb{Q}$-factoriality of dlt pairs in codim $=2)$. Let $(X, D)$ be a dlt pair. Then there exists a closed subset $Z \subset X$ with $\operatorname{codim}_{X} Z \geq 3$ such that $X \backslash Z$ is $\mathbb{Q}$-factorial.

Proof. Since every dlt pair is a limit of klt pairs, [KM98, Prop. 2.43], there exists a $\mathbb{Q}$ divisor $D^{\prime}$ on $X$ such that $\left(X, D^{\prime}\right)$ is klt. We may therefore assume from the beginning without loss of generality that $(X, D)$ is klt.

Applying [BCHM10, Cor. 1.4.3] with $\Delta_{0}=\Delta$ and $\mathfrak{E}=\emptyset$, we obtain a log-terminal model of $X$, i.e., a small birational morphism $p: Y \rightarrow X$ from a $\mathbb{Q}$-factorial variety $Y$ to $X$. Since $p$ is small, there exists a closed subset $Z \subset X$ with codimension $\operatorname{codim}_{X} Z \geq 3$ such that $p^{-1}: X \backslash Z \rightarrow Y \backslash p^{-1}(Z)$ is well-defined and an isomorphism. This finishes the proof.

Remark 9.2. Instead of using the full force of minimal model theory, it is certainly possible to give an elementary (though lengthy) proof that follows the arguments of [KM98, Lem. 4.10] after using repeated hyperplane sections to reduce to the surface case.

The reader who is willing to use the classification of dlt pairs over arbitrary, not necessarily closed, fields of characteristic zero might prefer the following argument, suggested by János Kollár.

Alternate proof of Proposition 9.1 using localization. Let $U \subseteq X$ be the maximal open set which is locally $\mathbb{Q}$-factorial, and set $Z=X \backslash U$. To prove Proposition 9.1 it suffices to show that $\operatorname{codim}_{X} Z \geq 3$. If not, let $Z^{\prime} \subseteq Z$ be a component of codimension $\operatorname{codim}_{X} Z^{\prime}=2$. Localisation at the generic point of $Z^{\prime}$ then gives a 2-dimensional local dlt pair, which is defined over a field of characteristic zero but which is not $\mathbb{Q}$-factorial. This contradicts [KM98, Prop. 4.11]. 
9.B. The local structure of canonical pairs in codimension 2. If $(X, D)$ is a canonical (or $\log$ canonical) pair and $x \in X$ a point, then the discrepancy of $(X, D)$ at $x$ is small if either $X$ is very singular at $x$ or if $D$ has high multiplicity at $x$. Conversely, it is true that $X$ cannot be very singular wherever the multiplicity of $D$ is large. This principle leads to the following description of canonical pairs along reduced components of the boundary divisor $D$.

Proposition 9.3 (Codimension 2 structure of canonical pairs along the boundary). Let $(X, D)$ be a canonical pair with $\lfloor D\rfloor \neq 0$. Then there exists a closed subset $Z \subset \operatorname{supp}\lfloor D\rfloor$ with $\operatorname{codim}_{X} Z \geq 3$ such that for any point $z \in(\operatorname{supp}\lfloor D\rfloor) \backslash Z$,

(9.3.1) the pair $(X, D)$ is snc at $z$, and

(9.3.2) the subvariety supp $D$ is smooth at $z$.

Proof. Consider general hyperplanes $H_{1}, \ldots, H_{\operatorname{dim} X-2} \subseteq X$ and set

$$
\left(H, D_{H}\right):=\left(H_{1} \cap \cdots \cap H_{\operatorname{dim} X-2}, D \cap H_{1} \cap \cdots \cap H_{\operatorname{dim} X-2}\right) .
$$

Lemma 2.24 then asserts that $\left(H, D_{H}\right)$ is a canonical surface pair. The classification of these pairs, [KM98, Thm. 4.5(2)], therefore applies to show that both $H$ and $\operatorname{supp} D_{H}$ are smooth along supp $\left\lfloor D_{H}\right\rfloor$. The Cutting-Down Lemma2.22 then gives that

- the properties (9.3)11 and 9.32) hold for all points $z \in \operatorname{supp}\left\lfloor D_{H}\right\rfloor$, and

- we have an equality of sets, $\operatorname{supp}\left\lfloor D_{H}\right\rfloor=\operatorname{supp}(\lfloor D\rfloor) \cap H$.

The claim then follows because the hyperplanes $H_{i}$ are generic.

9.C. The local structure of klt pairs in codimension 2. We show that the underlying space of a klt pair has quotient singularities in codimension 2. This result is used in Sections 10-12, where we reduce the study of reflexive differentials on singular spaces to the study of $G$-invariant differentials on suitable local Galois coverings with Galois group $G$.

Proposition 9.4 (Klt spaces have quotient singularities in codimension 2). Let $(X, D)$ be a klt pair. Then there exists a closed subset $Z \subset X$ with $\operatorname{codim}_{X} Z \geq 3$ such that $X \backslash Z$ has quotient singularities.

More precisely, every point $x \in X \backslash Z$ has an analytic neighbourhood that is biholomorphic to an analytic neighbourhood of the origin in a variety of the form $\mathbb{C}^{\operatorname{dim} X} / G$, where $G$ is a finite subgroup of $G L_{\operatorname{dim} X}(\mathbb{C})$ that does not contain any quasi-reflections. The quotient map is a finite Galois map, totally branched over the singular locus and étale outside of the singular set.

Remark 9.4.1. For families of du Val singularities, similar statements appear in the literature, e.g. in [Rei80, Cor. 1.14] or [Nam01, Proof of Prop. 1], but with little or no indication of proof. Our proof of Proposition 9.4 employs Grauert's miniversal deformation space for analytic germs of isolated singularities, tautness of dlt surface singularities and Teissier's "economy of the miniversal deformation", [Tei77]. We would like to thank Yujiro Kawamata and Gert-Martin Greuel for discussions on the subject.

The remainder of the present Section 9.C is devoted to a proof of Proposition 9.4. We subdivide the proof into a number of relatively independent steps.

9.C.1. Proof of Proposition 9.4: projection to the singular set. Observe that the assertion of Proposition 9.4 is local on $X$. Recalling from Proposition 9.1 that $X$ is $\mathbb{Q}$-factorial in codimension 2, observe that it suffices to prove Proposition 9.4 under the following additional assumption. 
Additional Assumption 9.5. The variety $X$ is $\mathbb{Q}$-factorial. In particular, we assume that the pair $(X, \emptyset)$ is klt, cf. [KM98, Cor. 2.39]. The singular locus $T:=X_{\text {sing }}$ is irreducible and of codimension $\operatorname{codim}_{X} T=2$.

Recall from Proposition 2.25 that there exists an open set $X^{\circ} \subseteq X$ such that $T^{\circ}:=$ $T \cap X^{\circ}$ is not empty, and a diagram

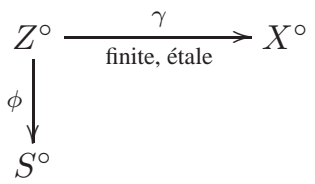

such that the restriction of $\phi$ to any connected component of $\gamma^{-1}\left(T^{\circ}\right)$ is an isomorphism. It is clear that $X$ is smooth at all points of $X \backslash\left(X^{\circ} \cup T\right)$, and that $\operatorname{codim}_{X}\left(T \backslash T^{\circ}\right) \geq 3$. Consequently, it suffices to prove Proposition 9.4 for points contained in $X^{\circ}$. Better still, since the assertion of Proposition 9.4 is local in the analytic topology, it suffices to prove Proposition 9.4 for the variety $Z^{\circ}$, even after removing all but one component of $\gamma^{-1}\left(T^{\circ}\right)$. We may therefore assume the following.

Additional Assumption 9.6. There exists a surjective morphism $\phi: X \rightarrow S$ with connected fibres whose restriction $\left.\phi\right|_{T}: T \rightarrow S$ is isomorphic.

Observation 9.7. Let $U \subseteq S$ be any Zariski-open subset. As in the previous paragraph, observe that $X$ is smooth at all points of $X \backslash\left(\phi^{-1}(U) \cup T\right)$, and that $\operatorname{codim}_{X} T \backslash \phi^{-1}(U) \geq$ 3. As above, we see that to prove Proposition 9.4, it suffices to consider the open set $\phi^{-1}(U) \subseteq X$ only.

Observation 9.7, together with the Generic Flatness Lemma, [FGI ${ }^{+}$05, Thm. 5.12], and the Cutting-Down Lemma 2.22 allows to assume the following.

Additional Assumption 9.8. The morphism $\phi$ is flat. Given any point $s \in S$, the preimage $X_{s}:=\phi^{-1}(s)$ is a normal klt surface 2 . If $t_{s} \in T$ is the unique point with $\phi\left(t_{s}\right)=s$, then $X_{s}$ is smooth away from $t_{s}$.

9.C.2. Proof of Proposition 9.4: simultaneous resolution of singularities. In this subsection, we aim to show that, possibly shrinking $S$ further, there exists a simultaneous minimal resolution of the surface singularities $\left(X_{s}\right)_{s \in S}$.

Claim 9.9. There exists a dense smooth open set $S^{\circ} \subseteq S$ with preimage $X^{\circ}:=\phi^{-1}\left(S^{\circ}\right)$, and a resolution of singularities $\pi: \widetilde{X}^{\circ} \rightarrow X^{\circ}$ such that the composition $\psi:=\phi \circ \pi$ is smooth over $S^{\circ}$, and such that the fibre $\widetilde{X}_{s}:=\psi^{-1}(s)$ is a minimal resolution of the klt surface $X_{s}$, for every $s \in S^{\circ}$.

Proof. To start, let $\pi: \widetilde{X} \rightarrow X$ be any resolution of singularities. If $s \in S$ is general, it is then clear that $\widetilde{X}_{s}$ is smooth. We may thus choose $S^{\circ}$ such that all scheme-theoretic fibres $\left(\widetilde{X}_{s}\right)_{s \in S^{\circ}}$ are smooth. Set $\widetilde{X}^{\circ}:=\widetilde{\phi}^{-1}\left(T^{\circ}\right)$.

Now, if $K_{\tilde{X}^{\circ} / X^{\circ}}$ is nef, then none of the surfaces $\widetilde{X}_{s}$ contains a $(-1)$-curve, $\pi$ is a simultaneous minimal resolution of the surface singularities $\left(X_{s}\right)_{s \in S^{\circ}}$, and the proof is complete.

\footnotetext{
${ }^{2}$ More precisely, we assume that the pair $\left(X_{s}, \emptyset\right)$ is klt.
} 
If $K_{\widetilde{X}^{\circ} / X^{\circ}}$ is not nef, then the Relative Cone Theorem, [KM98, Thm. 3.25] asserts that there exists a factorisation of $\pi$ via a birational, $\pi$-relative contraction of an extremal ray,

$$
\tilde{X}^{\circ} \underset{\pi_{1}}{\longrightarrow} \hat{X}^{\circ} \underset{\pi_{2}}{\longrightarrow} X^{\circ} \stackrel{\phi}{\longrightarrow} S^{\circ} \text {. }
$$

If $\pi_{1}$ is a divisorial contraction, then $\widehat{X}^{\circ}$ is terminal, [KM98, Cor. 3.43.(3)], and $\operatorname{codim}_{\widehat{X}^{\circ}} \widehat{X}_{\operatorname{sing}}^{\circ} \geq 3$, [KM98, Cor. 5.18]. If $\pi_{1}$ is a small contraction, it is likewise clear that $\operatorname{codim}_{\widehat{X}^{\circ}} \widehat{X}_{\text {sing }}^{\circ} \geq 3$. In either case, the singular set $\widehat{X}_{\text {sing }}^{\circ}$ does not dominate $S^{\circ}$. Replacing $S^{\circ}$ by a suitable subset, we may assume that $\pi_{2}: \widehat{X}^{\circ} \rightarrow X^{\circ}$ is a resolution of singularities with relative Picard-number $\rho\left(\widehat{X}^{\circ} / X^{\circ}\right)<\rho\left(\widetilde{X}^{\circ} / X^{\circ}\right)$. Replacing $\widetilde{X}^{\circ}$ by $\widehat{X}^{\circ}$ and repeating the process finitely many times, we will end up with a resolution where $K_{\tilde{X}^{\circ} / X^{\circ}}$ is nef. Claim 9.9 is thus shown.

Claim 9.9 and Observation 9.7 together allow to assume the following.

Additional Assumption 9.10. There exists a resolution of singularities $\pi: \widetilde{X} \rightarrow X$ such that the composition $\psi:=\phi \circ \pi$ is smooth, and such that for any $s \in S$, the fibre $\widetilde{X}_{s}:=$ $\psi^{-1}(s)$ is a minimal resolution of the klt surface singularity $X_{s}$.

9.C.3. Proof of Proposition 9.4: the isomorphism type of the surface germs $X_{s}$. Given a point $s \in S$, we consider the germ of the pointed surface $X_{s}$ at the point $t_{s} \in T$, the unique point of $T$ that satisfies $\phi\left(t_{s}\right)=s$. We use the symbol $\left(\mathbf{X}_{s} \ni t_{s}\right)$ to denote this germ.

Claim 9.11. There exists a dense Zariski-open subset $S^{\circ} \subseteq S$ such that for any two points $s_{1}, s_{2} \in S^{\circ}$, the associated germs of the pointed surfaces are isomorphic, $\left(\mathbf{X}_{s_{1}} \ni t_{s_{1}}\right) \simeq$ $\left(\mathbf{X}_{s_{2}} \ni t_{s_{2}}\right)$.

Proof. By [Ver76, Cor. 5.1], there exists a Zariski dense open subset $S^{\circ} \subseteq S$ with preimage $\tilde{X}^{\circ}:=\psi^{-1}\left(S^{\circ}\right)$ such that $\left.\psi\right|_{\tilde{X}^{\circ}}: \widetilde{X}^{\circ} \rightarrow S^{\circ}$ is a topological fibre bundle (in the analytic topology). As a consequence of the classification of log-terminal surface singularities, cf. [Kaw88, Thm. 9.6], the analytic isomorphism type of any such singularity is uniquely determined by the resolution graph (labelled with self-intersection numbers) of its minimal resolution. In other words, log terminal surface singularities are taut in the sense of Laufer [Lau73, Def. 1.1]. Since $\left.\psi\right|_{\widetilde{X}^{\circ}}$ is a fibre bundle, Claim 9.11 follows.

Again, Observation 9.7 allows to shrink $S$ and assume the following.

Additional Assumption 9.12. For any two points $s_{1}, s_{2} \in S$, we have an isomorphism $\left(\mathbf{X}_{s_{1}} \ni t_{s_{1}}\right) \simeq\left(\mathbf{X}_{s_{2}} \ni t_{s_{2}}\right)$.

9.C.4. Proof of Proposition 9.4: the completion of the proof. Let now $t \in T=X_{\operatorname{sing}}$ be any point, with image $s:=\phi(t)$. Note that by Assumption 9.8, the point $t$ is the unique singular point in the klt surface $X_{s}$. Since $\left(\mathbf{X}_{s} \ni t\right)$ is the germ of an isolated singularity, a theorem of Grauert, [Gra72], asserts the existence of a miniversal deformation space $(\mathbf{U} \ni 0)$ for $\left(\mathbf{X}_{s} \ni t\right)$, which is itself a germ of a pointed complex space; we refer to [GLS07, Sect. II.1] for these matters. Since $\phi: X \rightarrow S$ is flat, we obtain a holomorphic map of pointed space germs, say $\eta:(\mathbf{S} \ni s) \rightarrow(\mathbf{U} \ni 0)$. Since all fibres of $\phi$ give isomorphic space germs by Assumption 9.12 it follows from the "economy of the miniversal deformation", [HM89, Cor. 2], [Tei77, Thm. 4.8.4] that $\eta$ is the constant map which maps the germ $(\mathbf{T} \ni t)$ to $0 \in \mathbf{U}$. The universal property of the miniversal deformation space then gives an isomorphism of germs

$$
(\mathbf{X} \ni t) \simeq\left(\mathbf{S} \times \mathbf{X}_{s} \ni(s, t)\right) .
$$


Since $T$ and $S$ are smooth, there exists a neighbourhood $U$ of $t$ in $X$ such that $U$ is biholomorphic to $B^{\operatorname{dim} X-2} \times\left(X_{s} \cap U\right)$, where $B^{k}$ denotes the unit ball in $\mathbb{C}^{k}$. It follows from the classification of log terminal surface singularities and from the general description of quotient singularities, cf. [Kaw88, Thm. 9.6] and [Pri67], that the exits a finite group $G \subset G L_{2}(\mathbb{C})$ without quasi-reflections such that a neighbourhood of $t \in X_{s}$ is biholomorphic to a neighbourhood of the origin in $\mathbb{C}^{2} / G$. The quotient map is totally branched over the origin and étale elsewhere. Hence, $t \in X$ possesses a neighbourhood $U^{\prime} \subseteq U$ that is biholomorphic to a complex space of the form $\left(B^{\operatorname{dim} X-2} \times B^{2}\right) / G$, where $G$ is a finite group acting linearly and without quasi-reflections on the second factor, and where the quotient map is totally branched over the singular set and étale elsewhere.

9.D. The local structure of dit pairs in codimension 2. We conclude the present Section 9 by describing the codimension 2 structure of dlt pairs along the reduced components of the boundary, similarly to Proposition 9.3 above. Since dlt pairs are klt away from the reduced components of the boundary, [KM98, Prop. 2.41], Propositions 9.4 and 9.13 together give a full account of the structure of dlt pairs in codimension 2 . These results are summarised in Corollary 9.15 at the end of this section.

Proposition 9.13 (Codimension 2 structure of dlt pairs along the reduced boundary). Let $(X, D)$ be a dlt pair with $\lfloor D\rfloor \neq 0$. Then there exists a closed subset $Z \subset X$ with $\operatorname{codim}_{X} Z \geq 3$ such that $X \backslash Z$ is $\mathbb{Q}$-factorial, and such that for every point $x \in(\operatorname{supp}\lfloor D\rfloor) \backslash Z$ one of the following two conditions holds.

(9.13.1) The pair $(X, D)$ is snc at $x$, and the point $x$ is contained in precisely two components of $D$. These components have coefficient one in $D$ and and intersect transversely at $x$.

(9.13.2) The divisor $\lfloor D\rfloor$ is smooth at $x$ and the pair $(X, D)$ is plt at $x$.

As with Proposition 9.3 the proof of Proposition 9.13 relies on cutting-down and on classification results for surface pairs. Before starting with the proof, we recall the relevant classification of dlt surface pairs for the reader's convenience.

Fact 9.14 (Classification of dlt surface pairs, [KM98, Cor. 5.55]). Let $(X, D)$ be a dlt surface pair, and let $x \in \operatorname{supp}\lfloor D\rfloor$ be any point. Then either one of the following holds.

(9.14.1) The pair $(X, D)$ is snc at $x$, and $x$ is contained in precisely two components of $D$. These components have coefficient one and intersect transversely at $x$.

(9.14.2) The divisor $\lfloor D\rfloor$ is smooth at $x$.

With Fact 9.14 at hand, the proof of Proposition 9.13 becomes rather straightforward.

Proof of Proposition 9.13 To start, recall from Proposition 9.1 that $X$ is $\mathbb{Q}$-factorial in codimension 2 . Removing a suitable small subset, we may therefore assume without loss of generality that $X$ is $\mathbb{Q}$-factorial

Consider general hyperplanes $H_{1}, \ldots, H_{\operatorname{dim} X-2} \subseteq X$, and set

$$
\left(H, D_{H}\right):=\left(H_{1} \cap \cdots \cap H_{\operatorname{dim} X-2}, D \cap H_{1} \cap \cdots \cap H_{\operatorname{dim} X-2}\right) .
$$

Then 2.223 of the Cutting-Down Lemma 2.22 asserts that $\operatorname{supp}\left(\left\lfloor D_{H}\right\rfloor\right)=H \cap$ $\operatorname{supp}(\lfloor D\rfloor)$. By general choice of the $H_{i}$, it suffices to show that the properties 9.13]1] or 9.132 hold for all points $x \in \operatorname{supp}\left(\left\lfloor D_{H}\right\rfloor\right)$. Fix one such point for the remainder of the proof.

By Lemma 2.24 the surface pair $\left(H, D_{H}\right)$ is dlt, so that the classification stated in Fact 9.14 applies. If we are in case 9.1411, it follows from 2.225 and 2.22 of 
Lemma 2.22 that the pair $(X, D)$ is snc at $x$, and that near $x$ the pair $(X, D)$ is of the form stated in 9.1311.

We may thus assume that we are in case $9.14 \mid 2]$, where smoothness of $\lfloor D\rfloor$ at $x$ follows from (2.2214). The fact that pair $(X, D)$ is plt at $x$ follows from [KM98, Prop. 5.51].

Corollary 9.15. Let $(X, D)$ be a dlt pair. Then there exists a closed subset $Z \subset X$ with $\operatorname{codim}_{X} Z \geq 3$ such that $X^{\circ}:=X \backslash Z$ is $\mathbb{Q}$-factorial, and such that there exists a covering of $X^{\circ}$ by subsets $\left(U_{\alpha}\right)_{\alpha \in A}$ that are open in the analytic topology, and admit covering maps

$$
\gamma_{\alpha}: V_{\alpha} \rightarrow U_{\alpha} \text { finite Galois cover, étale in codimension one }
$$

such that the pairs $\left(V_{\alpha}, \gamma_{\alpha}^{*}\lfloor D\rfloor\right)$ are snc for all indices $\alpha \in A$. Furthermore, the covering may be chosen to satisfy the following additional conditions.

(9.15.1) Only finitely many of the open sets, say $U_{\alpha_{1}}, \ldots, U_{\alpha_{k}}$, intersect $\operatorname{supp}\lfloor D\rfloor$. The sets $U_{\alpha_{i}}$ are open in the Zariski topology, and the covering maps $\gamma_{\alpha_{i}}$ are algebraic morphisms of quasi-projective varieties.

(9.15.2) For any index $\alpha$ with $U_{\alpha} \cap \operatorname{supp}\lfloor D\rfloor=\emptyset$, the covering map $\gamma_{\alpha}$ is totally branched over the singular set, and étale elsewhere.

Remark 9.15.1. Since the $\gamma_{\alpha}$ are étale in codimension one, round-down of divisors commutes with pulling-back. That is, we have equalities $\gamma_{\alpha}^{*}\lfloor D\rfloor=\left\lfloor\gamma_{\alpha}^{*} D\right\rfloor$ for all $\alpha \in A$.

9.D.1. Proof of Corollary 9.15, setup of notation. Removing a subset of codimension 3, Proposition 9.13 allows to assume that the variety $X$ is $\mathbb{Q}$-factorial. In particular, we assume that the pair $(X,\lfloor D\rfloor)$ is likewise dlt, [KM98, Cor. 2.39]. We may therefore assume that $D$ is reduced, i.e., that $D=\lfloor D\rfloor$. Finally, consider the open set $X^{\prime}:=X \backslash \operatorname{supp} D$ and observe that the pair $\left(X^{\prime}, \emptyset\right)$ is klt, [KM98, Prop. 2.41].

The open cover $\left(U_{\alpha}\right)_{\alpha \in A}$ will be constructed in two steps, first covering $\operatorname{supp} D$ with finitely many Zariski-open sets, and then covering $X^{\prime}$ by (possibly infinitely many) sets that are open only in the analytic topology. In each step, we might need to remove from $X$ finitely many further sets of codimension 3 .

9.D.2. Proof of Corollary 9.15, covering supp D. Assuming that $D \neq 0$ and removing a suitable subset of codimension 3, we may assume that for all points $x \in \operatorname{supp} D$ either Condition 9.1311) or Condition 9.13/2) of Proposition 9.13 holds.

We start the construction setting $U_{1}:=(X, D)_{\text {reg }}$, and taking for $\gamma_{1}$ the identity map. Observing that $(X, D)$ is plt at all points of supp $D \backslash U_{1}$, we can cover supp $D \backslash U_{1}$ by finitely many affine Zariski-open subsets $U_{2}, \ldots, U_{k}$ such that the following holds for all indices $i$,

- the pairs $\left(U_{i}, D\right)$ are plt, and

- there are numbers $m_{i}>0$ and isomorphisms $\mathscr{O}_{U_{i}}\left(m_{i}\left(K_{X}+D\right)\right) \simeq \mathscr{O}_{U_{i}}$.

Let $\gamma_{i}: V_{i} \rightarrow U_{i}$ be the associated index-one covers, which are finite cyclic Galois covers that are étale in codimension one. Set $\Delta_{i}:=\gamma_{i}^{*} D$. Since discrepancies do not increase under this kind of covers, see [KM98, Prop. 5.20(3)], the pairs $\left(V_{i}, \Delta_{i}\right)$ are again plt, so the discrepancies of all exceptional divisors are greater than -1 . Better still, since the logcanonical divisors $K_{V_{i}}+\Delta_{i}$ are Cartier by construction, these discrepancies are integral, and therefore non-negative. The reduced pairs $\left(V_{i}, \Delta_{i}\right)$ are thus canonical. In this setup, Proposition 9.3 applies to show that there exists a subset $Z^{\prime} \subset X$ of $\operatorname{codim}_{X} Z^{\prime} \geq 3$ such that all pairs $\left(V_{i} \backslash \gamma^{-1}\left(Z^{\prime}\right), \Delta_{i} \backslash \gamma^{-1}\left(Z^{\prime}\right)\right)$ are snc. Removing the subset $Z^{\prime}$ from $X$, we obtain the desired covering. 
9.D.3. Proof of Corollary 9.15, covering most of $X^{\circ}$. Let $Z^{\prime \prime} \subset X^{\prime}$ be the subset of codimension 3 that is discussed in Proposition 9.4. Removing from $X$ the closure of $Z^{\prime \prime}$, the existence of the covering follows from the assertion that $X^{\prime}$ has quotient singularities of the form described in Proposition 9.4 and therefore $\gamma_{\alpha}$ is totally branched over the singular set.

\section{RELATIVE DifFERENTIAL SEQUENCES ON DLT PAIRS}

In this section we start the systematic study of sheaves of reflexive differentials on dlt pairs. Specifically we construct a standard exact sequence for forms of degree 1 with respect to a morphism $\phi: X \rightarrow T$ and study the induced filtration for forms of degree $p \geq 2$.

10.A. The relative differential sequence for snc pairs. Here we recall the generalisation of the standard sequence for relative differentials, [Har77, Prop. II.8.11], to the logarithmic setup. Let $(X, D)$ be a reduced snc pair, and $\phi: X \rightarrow T$ an snc morphism of $(X, D)$, as introduced in Definition 2.9. In this setting, the standard pull-back morphism of 1-forms extends to yield the following exact sequence of locally free sheaves on $X$,

$$
0 \rightarrow \phi^{*} \Omega_{T}^{1} \rightarrow \Omega_{X}^{1}(\log D) \rightarrow \Omega_{X / T}^{1}(\log D) \rightarrow 0,
$$

called the "relative differential sequence for logarithmic differentials". We refer to [EV90, Sect. 4.1] or [Del70, Sect. 3.3] for a more detailed explanation. For forms of higher degrees, the sequence 10.1 induces filtrations

$$
\Omega_{X}^{p}(\log D)=\mathscr{F}^{0}(\log ) \supseteq \mathscr{F}^{1}(\log ) \supseteq \cdots \supseteq \mathscr{F}^{p}(\log ) \supseteq\{0\}
$$

with quotients

$$
0 \rightarrow \mathscr{F}^{r+1}(\log ) \rightarrow \mathscr{F}^{r}(\log ) \rightarrow \phi^{*} \Omega_{T}^{r} \otimes \Omega_{X / T}^{p-r}(\log D) \rightarrow 0
$$

for all $r$. We refer to [Har77, Ex. II.5.16] for the construction of (10.2). For the reader's convenience, we recall without proof of the following elementary properties of the relative differential sequence.

Fact 10.4 (Composition with étale morphisms). Let $(X, D)$ be a reduced snc pair, and let $\phi: X \rightarrow T$ be an snc morphism of $(X, D)$. If $\gamma: Z \rightarrow X$ is an étale morphism, and $\Delta:=\gamma^{*}(D)$, then $\psi:=\phi \circ \gamma$ is an snc morphism of $(Z, \Delta)$, the natural pull-back map $d \gamma: \gamma^{*} \Omega_{X}^{1}(\log D) \rightarrow \Omega_{Z}^{1}(\log \Delta)$ is isomorphic, and induces isomorphisms between the pull-back of the filtration 10.2 induced by $\phi$, and the filtration $\widetilde{\mathscr{F}}^{r}(\log )$ of $\Omega_{Z}^{p}(\log \Delta)$ induced by the composition $\psi$,

$$
d \gamma\left(\gamma^{*} \mathscr{F}^{r}(\log )\right)=\widetilde{\mathscr{F}}^{r}(\log ), \quad \forall r
$$

Fact 10.5 (Compatibility with fiber-preserving groups actions). Let $G$ be a finite group which acts on $X$, with associated isomorphisms $\phi_{g}: X \rightarrow X$. Assume in addition that the $G$-action is fibre preserving, i.e., assume that $\phi \circ \phi_{g}=\phi$ for every $g \in G$. Then all sheaves that appear in Sequences (10.1) and (10.3) as well as in the filtration in 10.2) can naturally be endowed with $G$-sheaf structures. All the morphisms discussed above preserve this additional structure, i.e., they are morphisms of $G$-sheaves in the sense of Definition B.1 
10.B. Main result of this section. The main result of this section, Theorem 10.6, gives analogues of 10.1 -10.3) in case where $(X, D)$ is dit. In the absolute case Theorem 10.6 essentially says that all properties of the differential sequence discussed in Section 10.A still hold on a dlt pair $(X, D)$ if one removes from $X$ a set $Z$ of codimension at least 3 .

Theorem 10.6 (Relative differential sequence on dlt pairs). Let $(X, D)$ be a dlt pair with $X$ connected. Let $\phi: X \rightarrow T$ be a surjective morphism to a normal variety $T$. Then, there exists a non-empty smooth open subset $T^{\circ} \subseteq T$ with preimages $X^{\circ}=\phi^{-1}\left(T^{\circ}\right)$, $D^{\circ}=D \cap X^{\circ}$, and a filtration

$$
\Omega_{X^{\circ}}^{[p]}\left(\log \left\lfloor D^{\circ}\right\rfloor\right)=\mathscr{F}[0](\log ) \supseteq \cdots \supseteq \mathscr{F}^{[p]}(\log ) \supseteq\{0\}
$$

on $X^{\circ}$ with the following properties.

(10.6.2) The filtrations (10.2) and (10.6.1) agree wherever the pair $\left(X^{\circ},\left\lfloor D^{\circ}\right\rfloor\right)$ is snc, and $\phi$ is an snc morphism of $\left(X^{\circ},\left\lfloor D^{\circ}\right\rfloor\right)$.

(10.6.3) For any $r$, the sheaf $\mathscr{F}^{[r]}(\log )$ is reflexive, and $\mathscr{F}^{[r+1]}(\log )$ is a saturated subsheaf of $\mathscr{F}^{[r]}(\log )$.

(10.6.4) For any $r$, there exists a sequence of sheaves of $\mathscr{O}_{X \circ}$-modules,

$$
0 \rightarrow \mathscr{F}^{[r+1]}(\log ) \rightarrow \mathscr{F}^{[r]}(\log ) \rightarrow \phi^{*} \Omega_{T^{\circ}}^{r} \otimes \Omega_{X^{\circ} / T^{\circ}}^{[p-r]}\left(\log \left\lfloor D^{\circ}\right\rfloor\right) \rightarrow 0,
$$

which is exact and analytically locally split in codimension 2 .

(10.6.5) There exists an isomorphism $\mathscr{F}[p](\log ) \simeq \phi^{*} \Omega_{T^{\circ}}^{p}$.

Remark 10.6.6. To construct the filtration in 10.6.1), one takes the filtration 10.2) which exists on the open set $X \backslash X_{\text {sing }}$ wherever the morphism $\phi$ is snc, and extends the sheaves to reflexive sheaves that are defined on all of $X$. It is then not very difficult to show that the sequences 10.64) are exact and locally split away from a subset $Z \subset X$ of codimension $\operatorname{codim}_{X} Z \geq 2$. The main point of Theorem 10.6 is, however, that it suffices to remove from $X$ a set of codimension $\operatorname{codim}_{X} Z \geq 3$.

Before proving Theorem 10.6 in Section 10.C below, we first draw an important corollary. The assertion that Sequences 10.614 are exact and locally split away from a set of codimension three plays a pivotal role here.

Corollary 10.7 (Restriction of the relative differentials sequence to boundary components). In the setup of Theorem 10.6 assume that $\lfloor D\rfloor \neq 0$ and let $D_{0} \subseteq \operatorname{supp}\lfloor D\rfloor$ be any irreducible component that dominates $T$. Recall that $D_{0}$ is normal, [KM98, Cor. 5.52].

If $r$ is any number, then Sequences (10.614) induce exact sequences of reflexive sheaves on $D_{0}^{\circ}:=D_{0} \cap X^{\circ}$, as follow ${ }^{3}$

$$
\left.\left.\left.0 \rightarrow \mathscr{F}^{[r+1]}(\log )\right|_{D_{0}^{\circ}} ^{* *} \rightarrow \mathscr{F} \mathscr{F}^{[r]}(\log )\right|_{D_{0}^{\circ}} ^{* *} \rightarrow \phi^{*} \Omega_{T}^{r} \otimes \Omega_{X^{\circ} / T^{\circ}}^{[p-r r}\left(\log \left\lfloor D^{\circ}\right\rfloor\right)\right|_{D_{0}^{\circ}} ^{* *}
$$

Proof. Since $D_{0}$ is normal, there exists a subset $Z \subset X^{\circ}$ with $\operatorname{codim}_{X} \circ Z \geq 3$ such that - the divisor $D_{0}^{\circ}:=D_{0} \cap X^{\circ}$ is smooth away from $Z$, and

- the Sequences (10.644) are exact and locally split away from $Z$.

It follows from the local splitting of (10.64) that the sequence obtained by restriction,

$$
\left.\left.\left.0 \rightarrow \mathscr{F}^{[r+1]}(\log )\right|_{D_{0}^{\circ} \backslash Z} \rightarrow \mathscr{F}^{[r]}(\log )\right|_{D_{0}^{\circ} \backslash Z} \rightarrow \phi^{*} \Omega_{T}^{r} \otimes \Omega_{X^{\circ} / T^{\circ}}^{[p-r]}\left(\log \left\lfloor D^{\circ}\right\rfloor\right)\right|_{D_{0}^{\circ} \backslash Z} \rightarrow 0,
$$

\footnotetext{
${ }^{3}$ For brevity of notation, we write $\left.\mathscr{F}[r](\log )\right|_{D_{0}} ^{* *}$ and $\left.\phi^{*} \Omega_{T}^{r} \otimes \Omega_{X / T}^{[p-r]}\left(\log D_{0}\right)\right|_{D_{0}} ^{* *}$ instead of the more correct forms $\left(\left.\mathscr{F}[r](\log )\right|_{D_{0}}\right)^{* *}$ and $\left.\phi^{*} \Omega_{T}^{r}\right|_{D_{0}} \otimes_{\mathscr{O}_{D_{0}}}\left(\left.\Omega_{X / T}^{[p-r]}\left(\log D_{0}\right)\right|_{D_{0}}\right)^{* *}$ here and throughout.
} 
is still exact. The exactness of 10.7.1) follows when one recalls that the functor which maps a sheaf to its double dual can be expressed in terms of a push-forward map and is therefore exact on the left.

10.C. Proof of Theorem 10.6. We prove Theorem 10.6 in the remainder of Section 10,

10.C.1. Proof of Theorem 10.6, setup and start of proof. By Remark2.11 we are allowed to make the following assumption without loss of generality.

Additional Assumption 10.8. The divisor $D \cap(X, D)_{\text {reg }}$ is relatively snc over $T$. In particular, $T$ is smooth, and the restriction of $\phi$ to the smooth locus $X_{\text {reg }}$ of $X$ is a smooth morphism.

As we have seen in Section 10.A the morphism $\phi: X \rightarrow T$ induces on the open set $(X, D)_{\text {reg }} \subseteq X$ a filtration of $\Omega_{(X, D)_{\text {reg }}^{p}}(\log \lfloor D\rfloor)$ by locally free saturated subsheaves, say $\mathscr{F}_{\circ}^{r}(\log )$. Let $i:(X, D)_{\text {reg }} \rightarrow X$ be the inclusion map and set

$$
\mathscr{F}^{[r]}(\log ):=i_{*}\left(\mathscr{F}_{\circ}^{r}(\log )\right) \text {. }
$$

We will then obtain a filtration as in 10.6.1). Notice that all sheaves $\mathscr{F}^{[r]}(\log )$ are saturated in $\Omega_{X}^{[p]}(\log \lfloor D\rfloor)$ since $\mathscr{F}_{\circ}^{r}(\log )$ is saturated in $\Omega_{(X, D)_{\text {reg }}^{p}}^{p}(\log \lfloor D\rfloor)$, cf. [OSS80, Lem. 1.1.16]. This shows the properties (10.612 and (10.6/3).

Using that push-forward is a left-exact functor, we also obtain exact sequences of reflexive sheaves on $X$ as follows,

$$
0 \rightarrow \mathscr{F}^{[r+1]}(\log ) \rightarrow \mathscr{F}^{[r]}(\log ) \rightarrow \phi^{*} \Omega_{T}^{r} \otimes \Omega_{X / T}^{[p-r]}(\log \lfloor D\rfloor) .
$$

We have to check that 10.8.1) is also right exact and locally split in codimension 2 , in the analytic topology. For this we will compare the sheaves just defined with certain $G$ invariant push-forward sheaves of local index-one covers. Once this is shown, the property 10.65) will follow automatically.

10.C.2. Proof of Theorem 10.6, simplifications. We use the description of the local structure of dlt pairs in codimension 2, done in Chapter 9 to simplify our situation.

The assertion of Theorem 10.6 is local. Since the sheaves $\mathscr{F}[\bullet[(\log )$ are reflexive, and since we only claim right-exactness of (10.8.1) in codimension 2 , we are allowed to remove subsets of codimension greater than or equal to 3 in $X$. We will use this observation to make a number of reduction steps.

Recall from Proposition 9.1 that $X$ is $\mathbb{Q}$-factorial in codimension 2, and hence the pair $(X,\lfloor D\rfloor)$ is dlt in codimension 2, see [KM98, Cor. 2.39 (1)]. This justifies the following.

Additional Assumption 10.9. The variety $X$ is $\mathbb{Q}$-factorial, and the boundary divisor $D$ is reduced, that is, $D=\lfloor D\rfloor$.

Corollary 9.15 allows us to assume the following.

Additional Assumption 10.10. There exists a cover $X=\cup_{\alpha \in A} U_{\alpha}$ by open subsets and there are finite morphisms $\gamma_{\alpha}: V_{\alpha} \rightarrow U_{\alpha}$, as described in Corollary 9.15

10.C.3. Proof of Theorem 10.6, study of composed morphisms. In Section 10.C.4 we study the sequence 10.8.1 by pulling it back to the smooth spaces $V_{\alpha}$, and by discussing relative differential sequences associated with the compositions $\psi_{\alpha}:=\phi \circ \gamma_{\alpha}$. We will show in this section that we may assume without loss of generality that these maps are snc morphisms of the pairs $\left(V_{\alpha}, \gamma_{\alpha}^{*} D\right)$.

Shrinking $T$, if necessary, and removing from $X$ a further set of codimension 3 , the following will hold. 
Additional Assumption 10.11. The singular locus $X_{\text {sing }}$ (with its reduced structure) is smooth, and so is the restriction $\left.\phi\right|_{X_{\text {sing }}}$.

Additional Assumption 10.12. If $\alpha \in A$ is one of the finitely many indices for which $U_{\alpha} \cap \operatorname{supp} D \neq \emptyset$, then the composition $\psi_{\alpha}:=\phi \circ \gamma_{\alpha}$ is an snc morphism of the pair $\left(V_{\alpha}, \gamma_{\alpha}^{*} D\right)$.

As a matter of fact, Assumptions 10.8 and 10.11 guarantee that all pairs $\left(V_{\alpha}, \gamma_{\alpha}^{*} D\right)$ are relatively snc over $T$, not just for those indices $\alpha \in A$ where $U_{\alpha}$ intersects $\operatorname{supp} D$ :

Claim 10.13. If $\alpha \in A$ is any index, then the composition $\psi_{\alpha}:=\phi \circ \gamma_{\alpha}$ is an snc morphism of the pair $\left(V_{\alpha}, \gamma_{\alpha}^{*} D\right)$.

Proof. Let $\alpha \in A$. If $U_{\alpha} \cap \operatorname{supp} D \neq \emptyset$, then Claim 10.13 follows directly from Assumption 10.12 and there is nothing to show. Otherwise, we have $\gamma_{\alpha}^{*} D=0$. Claim 10.13 will follow once we show that $\psi_{\alpha}: V_{\alpha} \rightarrow T$ has maximal rank at all points $v \in V_{\alpha}$. We consider the cases where $\gamma_{\alpha}(v)$ is a smooth, (resp. singular) point of $X$ separately.

If $\gamma_{\alpha}(v)$ is a smooth point of $X$, then 9.1512) of Corollary 9.15] asserts that $\gamma_{\alpha}$ is étale at $v$. Near $v$, the morphism $\psi_{\alpha}$ is thus a composition of an étale and a smooth map, and therefore of maximal rank.

If $\gamma_{\alpha}(v)$ is a singular point of $X$, consider the preimage $\Sigma:=\gamma_{\alpha}^{-1}\left(X_{\text {sing }}\right)$ with its reduced structure, and observe that $v \in \Sigma$. In this setting, (9.15]2) of Corollary 9.15asserts that $\gamma_{\alpha}$ is totally branched along $\Sigma$. In particular, the restriction $\left.\gamma_{\alpha}\right|_{\Sigma}: \Sigma \rightarrow X_{\text {sing }}$ is isomorphic and thus of maximal rank. By Assumption 10.11, the restriction $\left.\psi_{\alpha}\right|_{\Sigma}: \Sigma \rightarrow T$ is thus a composition of two morphisms with maximal rank, and has therefore maximal rank itself. It follows that $\psi_{\alpha}: V_{\alpha} \rightarrow T$ has maximal rank at $v$.

Right-exactness of the sequence 10.8.1) and its local splitting are properties that can be checked locally in the analytic topology on the open subsets $U_{\alpha}$. To simplify notation, we replace $X$ by one of the $U_{\alpha}$. Claim 10.13 and Additional Assumption 10.12 then allow to assume the following.

Additional Assumption 10.14. There exists a smooth manifold $Z$, endowed with an action of a finite group $G$ and associated quotient map $\gamma: Z \rightarrow X$. The cycle-theoretic preimage $\Delta:=\gamma^{*}(D)$ is a reduced snc divisor. Furthermore, the quotient map $\gamma$ is étale in codimension one, and the composition of $\psi:=\phi \circ \gamma: Z \rightarrow T$ is an snc morphism of the pair $(Z, \Delta)$.

10.C.4. Proof of Theorem 10.6 right-exactness of 10.8.1). Since $\psi$ is a $G$-invariant snc morphism between of the pair $(Z, \Delta)$, Fact 10.5 yields a filtration of $\Omega_{Z}^{p}(\log \Delta)$ by locally free $G$-subsheaves $\widetilde{\mathscr{F}}^{r}(\log )$ and $G$-equivariant exact sequences,

$$
0 \rightarrow \widetilde{\mathscr{F}}^{r+1}(\log ) \rightarrow \widetilde{\mathscr{F}}^{r}(\log ) \rightarrow \psi^{*} \Omega_{T}^{r} \otimes \Omega_{Z / T}^{p-r}(\log \Delta) \rightarrow 0 .
$$

By the Reflexivity LemmaB.4 the $G$-invariant push-forward-sheaves $\gamma_{*} \widetilde{\mathscr{F}}^{r}(\log )^{G}$ are then reflexive. By the Exactness LemmaB.3 these reflexive sheaves fit into the following exact sequences

(10.14.2) $0 \rightarrow \gamma_{*} \widetilde{\mathscr{F}}^{r+1}(\log )^{G} \rightarrow \gamma_{*} \widetilde{\mathscr{F}}^{r}(\log )^{G} \rightarrow \gamma_{*}\left(\psi^{*} \Omega_{T}^{r} \otimes \Omega_{Z / T}^{p-r}(\log \Delta)\right)^{G} \rightarrow 0$.

Since $\gamma$ is étale in codimension one, Fact 10.4 implies that the differential $d \gamma$ induces isomorphisms

$$
\mathscr{F}^{[r]}(\log ) \stackrel{\simeq}{\rightarrow} \gamma_{*} \widetilde{\mathscr{F}}^{r}(\log )^{G} .
$$


Furthermore, since $\psi=\phi \circ \gamma$, since $\Omega_{T}^{r}$ is locally free, and since $G$ acts trivially on $T$, it follows from the projection formula that there exist isomorphisms

$$
\begin{aligned}
\phi^{*} \Omega_{T}^{r} \otimes \Omega_{X / T}^{[p-r]}(\log D) & \stackrel{\simeq}{\rightarrow} \phi^{*} \Omega_{T}^{r} \otimes \gamma_{*} \Omega_{Z / T}^{p-r}(\log D)^{G} \\
& \simeq \gamma_{*}\left(\psi^{*} \Omega_{T}^{r} \otimes \Omega_{Z / T}^{p-r}(\log D)\right)^{G} .
\end{aligned}
$$

In summary, we note that the isomorphisms 10.14.3-10.14.5) make the following diagram commutative:

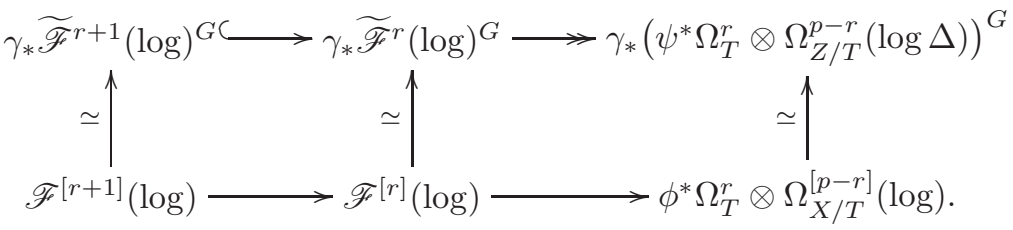

This shows that (10.8.1) is also right-exact, as claimed in (10.614).

10.C.5. Proof of Theorem 10.6 existence of local analytic splittings. It remains to show that 10.8.1) admits local analytic splittings in codimension 2 . This follows directly from the Splitting Lemma B.5, concluding the proof of Theorem 10.6

\section{RESIDUE SEQUENCES FOR REFLEXIVE DIFFERENTIAL FORMS}

A very important feature of logarithmic differentials is the existence of a residue map. In its simplest form consider a smooth hypersurface $D \subset X$ in a manifold $X$. The residue map is then the cokernel map in the exact sequence

$$
0 \rightarrow \Omega_{X}^{1} \rightarrow \Omega_{X}^{1}(\log D) \rightarrow \mathcal{O}_{D} \rightarrow 0 .
$$

In Section 11.A we first recall the general situation for an snc pair, for forms of arbitrary degree and in a relative setting. A generalisation to dlt pairs is the established in Sections 11.B 11.Cbelow. Without the dlt assumption, residue maps fail to exist in general.

11.A. Residue sequences for snc pairs. Let $(X, D)$ be a reduced snc pair. Let $D_{0} \subseteq D$ be an irreducible component and recall from [EV92, 2.3(b)] that there exists a residue sequence,

$$
0 \rightarrow \Omega_{X}^{p}\left(\log \left(D-D_{0}\right)\right) \longrightarrow \Omega_{X}^{p}(\log D) \stackrel{\rho^{p}}{\longrightarrow} \Omega_{D_{0}}^{p-1}\left(\log D_{0}^{c}\right) \rightarrow 0,
$$

where $D_{0}^{c}:=\left.\left(D-D_{0}\right)\right|_{D_{0}}$ denotes the "restricted complement" of $D_{0}$. More generally, if $\phi: X \rightarrow T$ is an snc morphism of $(X, D)$ we have a relative residue sequence

$$
0 \rightarrow \Omega_{X / T}^{p}\left(\log \left(D-D_{0}\right)\right) \longrightarrow \Omega_{X / T}^{p}(\log D) \stackrel{\rho^{p}}{\longrightarrow} \Omega_{D_{0} / T}^{p-1}\left(\log D_{0}^{c}\right) \rightarrow 0 .
$$

The sequence (11.1) is not a sequence of locally free sheaves on $X$, and its restriction to $D_{0}$ will never be exact on the left. However, an elementary argument, cf. [KK08, Lem. 2.13.2], shows that restriction of (11.1) to $D_{0}$ induces the following exact sequence

$$
\left.0 \rightarrow \Omega_{D_{0} / T}^{p}\left(\log D_{0}^{c}\right) \stackrel{i^{p}}{\rightarrow} \Omega_{X / T}^{p}(\log D)\right|_{D_{0}} \stackrel{\rho_{D}^{p}}{\longrightarrow} \Omega_{D_{0}}^{p-1}\left(\log D_{0}^{c}\right) \rightarrow 0,
$$

which is very useful for inductive purposes. We recall without proof several elementary facts about the residue sequence.

Fact 11.3 (Residue map as a test for logarithmic poles). If $\sigma \in H^{0}\left(X, \Omega_{X / T}^{p}(\log D)\right)$ is any reflexive form, then $\sigma \in H^{0}\left(X, \Omega_{X / T}^{p}\left(\log \left(D-D_{0}\right)\right)\right)$ if and only if $\rho^{p}(\sigma)=0$. 
Fact 11.4. In the simple case where $T$ is a point, $p=1$ and $D=D_{0}$, the restricted residue sequence (11.2) reads

$$
\left.0 \rightarrow \Omega_{D}^{1} \stackrel{i^{1}}{\rightarrow} \Omega_{X}^{1}(\log D)\right|_{D} \stackrel{\rho_{D}^{1}}{\longrightarrow} \mathscr{O}_{D} \rightarrow 0 .
$$

The sheaf morphisms $i^{1}$ and $\rho_{D}^{1}$ are then described as follows. If $V \subseteq X$ is any open set, and if $f \in \mathscr{O}_{X}(V)$ is a function that vanishes only along $D$, then

$$
\rho_{D}^{1}((d \log f) \mid D)=\operatorname{ord}_{D} f \cdot \mathbf{1}_{D \cap V},
$$

where $\mathbf{1}_{D \cap V}$ is the constant function with value one. If $g \in \mathscr{O}_{X}(V)$ is any function, then

$$
i^{1}\left(d\left(\left.g\right|_{D \cap V}\right)\right)=\left.(d g)\right|_{D \cap V} .
$$

Fact 11.5 (Base change property of the residue map). Let $(X, D)$ be a reduced snc pair, and $\pi: \widetilde{X} \rightarrow X$ a surjective morphism such that the pair $(\widetilde{X}, \widetilde{D})$ is snc, where $\widetilde{D}:=$ $\operatorname{supp} \pi^{*} D$. If $\widetilde{D}_{0} \subset \pi^{-1}\left(D_{0}\right)$ is any irreducible component, then there exists a diagram

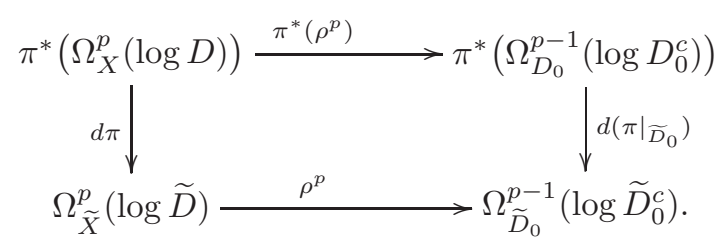

Fact 11.6 (Compatibility with fiber-preserving groups actions). Let $G$ be a finite group which acts on $X$, with associated isomorphisms $\phi_{g}: X \rightarrow X$. Assume that the $G$-action stabilises both the divisor $D$, and the component $D_{0} \subseteq D$, and assume that the action is fibre preserving, that is $\phi \circ \phi_{g}=\phi$ for every $g \in G$. Then all sheaves that appear in Sequences (11.1) and 111.2) are $G$-sheaves, in the sense of Definition B.1 on page 65, and all morphisms that appear in (11.1) and (11.2) are morphisms of $G$-sheaves.

11.B. Main result of this section. If the pair $(X, D)$ is not snc, no residue map exists in general. However, if $(X, D)$ is dlt, then [KM98, Cor. 5.52] applies to show that $D_{0}$ is normal, and an analogue of the residue map $\rho^{p}$ exists for sheaves of reflexive differentials, as we will show now.

To illustrate the problem we are dealing with, consider a normal space $X$ that contains a smooth Weil divisor $D=D_{0}$, similar to the one sketched in Figure 1 on page 13 One can easily construct examples where the singular set $Z:=X_{\operatorname{sing}}$ is contained in $D$ and has codimension 2 in $X$, but codimension one in $D$. In this setting, a reflexive form $\sigma \in H^{0}\left(D_{0},\left.\Omega_{D_{0}}^{[p]}\left(\log D_{0}\right)\right|_{D_{0}}\right)$ is simply the restriction of a form defined outside of $Z$, and the form $\rho^{[p]}(\sigma)$ is the extension of the well-defined form $\rho^{p}\left(\left.\sigma\right|_{D_{0} \backslash Z}\right)$ over $Z$, as a rational form with poles along $Z \subset D_{0}$. If the singularities of $X$ are bad, it will generally happen that the extension $\rho^{[p]}(\sigma)$ has poles of arbitrarily high order. Theorem 11.7 asserts that this does not happen when $(X, D)$ is dlt.

Theorem 11.7 (Residue sequences for dlt pairs). Let $(X, D)$ be a dlt pair with $\lfloor D\rfloor \neq \emptyset$ and let $D_{0} \subseteq\lfloor D\rfloor$ be an irreducible component. Let $\phi: X \rightarrow T$ be a surjective morphism to a normal variety $T$ such that the restricted map $\left.\phi\right|_{D_{0}}: D_{0} \rightarrow T$ is still surjective. Then, there exists a non-empty open subset $T^{\circ} \subseteq T$, such that the following holds if we denote the preimages as $X^{\circ}=\phi^{-1}\left(T^{\circ}\right), D^{\circ}=\bar{D} \cap X^{\circ}$, and the "complement" of $D_{0}^{\circ}$ as $D_{0}^{\circ, c}:=\left.\left(\left\lfloor D^{\circ}\right\rfloor-D_{0}^{\circ}\right)\right|_{D_{0}^{\circ}}$. 


\section{(11.7.1) There exists a sequence}

$$
\begin{aligned}
0 \rightarrow \Omega_{X^{\circ} / T^{\circ}}^{[r]}\left(\log \left(\left\lfloor D^{\circ}\right\rfloor-D_{0}^{\circ}\right)\right) \rightarrow \Omega_{X^{\circ} / T^{\circ}}^{[r]}\left(\log \left\lfloor D^{\circ}\right\rfloor\right) \\
\quad \stackrel{\rho^{[r]}}{\longrightarrow} \Omega_{D_{0}^{\circ} / T^{\circ}}^{[r-1]}\left(\log D_{0}^{\circ, c}\right) \rightarrow 0
\end{aligned}
$$

which is exact in $X^{\circ}$ outside a set of codimension at least 3 . This sequence coincides with the usual residue sequence (11.1) wherever the pair $\left(X^{\circ}, D^{\circ}\right)$ is snc and the map $\phi^{\circ}: X^{\circ} \rightarrow T^{\circ}$ is an snc morphism of $\left(X^{\circ}, D^{\circ}\right)$.

(11.7.2) The restriction of Sequence (11.7) to $D_{0}$ induces a sequence

$$
\begin{aligned}
0 \rightarrow \Omega_{D_{0}^{\circ} / T^{\circ}}^{[r]}\left(\log D_{0}^{\circ, c}\right) \rightarrow \Omega_{X^{\circ} / T^{\circ}}^{[r]}\left(\log \left\lfloor D^{\circ}\right\rfloor\right) & \left.\right|_{D_{0}^{\circ}} ^{* *} \\
& \stackrel{\rho_{D_{0}^{\circ}}^{[r]}}{\longrightarrow} \Omega_{D_{0}^{\circ} / T^{\circ}}^{[r-1]}\left(\log D_{0}^{\circ, c}\right) \rightarrow 0
\end{aligned}
$$

which is exact on $D_{0}^{\circ}$ outside a set of codimension at least 2 and coincides with the usual restricted residue sequence (11.2) wherever the pair $\left(X^{\circ}, D^{\circ}\right)$ is snc and the map $\phi^{\circ}: X^{\circ} \rightarrow T^{\circ}$ is an snc morphism of $\left(X^{\circ}, D^{\circ}\right)$.

Fact 11.3 and Theorem 11.7 together immediately imply that the residue map for reflexive differentials can be used to check if a reflexive form has logarithmic poles along a given boundary divisor.

Remark 11.8 (Residue map as a test for logarithmic poles). In the setting of Theorem 11.7 if $\sigma \in H^{0}\left(X, \Omega_{X}^{[p]}(\log \lfloor D\rfloor)\right)$ is any reflexive form, then $\sigma \in H^{0}\left(X, \Omega_{X}^{[p]}\left(\log \lfloor D\rfloor-D_{0}\right)\right)$ if and only if $\rho^{[p]}(\sigma)=0$.

11.C. Proof of Theorem 11.7 We prove Theorem 11.7 in the remainder of the present chapter. As in the setup of Theorem 10.6, discussed in Remark10.6.6, it is not difficult to construct Sequences (11.7/1) and 111.7/2) and to prove exactness outside a set of codimension 2 , but the main point is the exactness outside a set of codimension at least 3 .

11.C.1. Proof of Theorem 11.7 simplifications. Again, as in Section 10.C.2 we use the description of the codimension 2 structure of dlt pairs, obtained in Chapter 9 to simplify our situation. Since all the sheaves appearing in Sequences (11.711) and 11.7/2) are reflexive, it suffices to construct the sheaf morphism $\rho^{[r]}$ outside a set of codimension at least 3 . Notice also that existence and exactness of (11.7/1) and 111.7/2) are clear at all points in $(X, D)_{\text {reg }}$ where $\phi$ is an snc morphism of $(X, D)$. We will use these two observations to make a number of reduction steps.

As in Section 10.C.2, removing from $X$ a set of codimension 3, we may assume the following without loss of generality.

Additional Assumption 11.9. The variety $X$ is $\mathbb{Q}$-factorial, and the boundary divisor $D$ is reduced, that is, $D=\lfloor D\rfloor$.

Since the target of the residue map is a sheaf supported on $D$, we may work locally in a neighbourhood of $D$. Removing a further set of codimension more than 2, Corollary 9.15 therefore allows to assume the following.

Additional Assumption 11.10. There exists a cover $X=\cup_{\alpha \in A} U_{\alpha}$ by a finite number of affine Zariski open subsets $U_{\alpha}$ of $X$, and there exist finite Galois covers $\gamma_{\alpha}: V_{\alpha} \rightarrow U_{\alpha}$, étale in codimension one, such that the pairs $\left(V_{\alpha}, \gamma_{\alpha}^{*} D\right)$ are snc for all indices $\alpha$. 
Observe that the construction of the desired map $\rho^{[p]}$ can be done on the open subsets $U_{\alpha}$, once we have established the claim that the local maps constructed on the $U_{\alpha}$ coincide with the usual residue maps wherever this makes sense. To simplify notation, we will hence replace $X$ by one of the $U_{\alpha}$, writing $\gamma:=\gamma_{\alpha}, Z:=U_{\alpha}$ and $\Delta:=\gamma^{*} D$. The Galois group of $\gamma$ will be denoted by $G$. Shrinking $T$ if necessary, we may suppose the following.

Additional Assumption 11.11. The restriction of $\phi$ to the snc locus $(X, D)_{\text {reg }}$ is an snc morphism of $(X, D)$. The composition $\psi:=\phi \circ \gamma$ is an snc morphism of $(Z, \Delta)$.

With Assumption 11.11 in place, and the assertion of Theorem 11.7 being clear near points where $(X, D)$ is snc, the description of the codimension 2 structure of dlt pairs along the boundary, Proposition 9.13 allows us to assume the following.

Additional Assumption 11.12. The pair $(X, D)$ is plt. The divisors $D \subset X$ and $\Delta \subset Z$ are smooth and irreducible. In particular, we have $D=D_{0},\lfloor D\rfloor-D_{0}=0, D_{0}^{c}=0$, and the restricted maps $\left.\psi\right|_{\Delta}: \Delta \rightarrow T$ and $\left.\phi\right|_{D}: D \rightarrow T$ are smooth morphisms of smooth varieties.

11.C.2. Proof of Theorem 11.7, construction and exactness of (11.7.1). Since $\psi: Z \rightarrow T$ is an snc morphism of $(Z, \Delta)$, and since the irreducible divisor $\Delta \subset Z$ is invariant under the action of $G$, Fact 11.6 and the standard residue sequence 11.1 yield an exact sequence of morphisms of $G$-sheaves, as follows

$$
0 \rightarrow \Omega_{Z / T}^{r} \rightarrow \Omega_{Z / T}^{r}(\log \Delta) \stackrel{\rho^{r}}{\longrightarrow} \Omega_{\widetilde{D} / T}^{r-1} \rightarrow 0
$$

Recalling from Lemma B.3 that $\gamma_{*}(\cdot)^{G}$ is an exact functor, this induces an exact sequence of morphisms of $G$-sheaves, for the trivial $G$-action on $X$,

$$
0 \rightarrow \gamma_{*}\left(\Omega_{Z / T}^{r}\right)^{G} \rightarrow \gamma_{*}\left(\Omega_{Z / T}^{r}(\log \Delta)\right)^{G} \stackrel{\gamma_{*}\left(\rho^{r}\right)^{G}}{\longrightarrow} \gamma_{*}\left(\Omega_{\Delta / T}^{r-1}\right)^{G} \rightarrow 0 .
$$

Recall from Lemma B.4 that all the sheaves appearing in 11.12.1 are reflexive. The fact that $\gamma$ is étale in codimension one then implies that the pull-back of reflexive forms via $\gamma$ induces isomorphisms

$$
\begin{aligned}
& \Omega_{X / T}^{[r]} \stackrel{\simeq}{\longrightarrow} \gamma_{*}\left(\Omega_{Z / T}^{r}\right)^{G} \quad \text { and } \\
& \Omega_{X / T}^{[r]}(\log D) \stackrel{\simeq}{\longrightarrow} \gamma_{*}\left(\Omega_{Z / T}^{r}(\log \Delta)\right)^{G} .
\end{aligned}
$$

It remains to describe the last term of 11.12.1.

Claim 11.13. The restriction of $\gamma$ to $\Delta$ induces an isomorphism $\gamma_{*}\left(\Omega_{\Delta / T}^{r-1}\right)^{G} \simeq \Omega_{D / T}^{r-1}$.

Proof. By Assumption 11.12, the restricted morphism $\left.\gamma\right|_{\Delta}: \Delta \rightarrow D$ is a finite morphism of smooth spaces. The branch locus $S \subset D$ and the ramification locus $\widetilde{S} \subset \Delta$ are therefore both of pure codimension one.

The pull-back map of differential forms associated with $\left.\gamma\right|_{\Delta}$ yields an injection $\Omega_{D / T}^{r-1} \hookrightarrow \gamma_{*}\left(\Omega_{\Delta / T}^{r-1}\right)^{G}$. To prove Claim 11.13, it remains to show surjectivity. To this end, recall from Assumption 11.10 that $D$ and $\Delta$ are affine, and let $\sigma \in H^{0}\left(\Delta, \Omega_{\Delta}^{r-1}\right)^{G}$ be any $G$-invariant $(r-1)$-form on $\Delta$. Then there exists a rational differential form $\tau$ on $D$, possibly with poles along the divisor $S \subset D$ satisfying the relation

$$
\left.\left(\left.\gamma\right|_{\Delta}\right)^{*}(\tau)\right|_{\Delta \backslash \widetilde{S}}=\left.\sigma\right|_{\Delta \backslash \widetilde{S}}
$$

Recalling that regularity of differential forms can be checked on any finite cover, GKK10, Cor. 2.12.ii], Equation 11.13.1 implies that $\tau$ is in fact a regular form on $D$, that is, $\tau \in H^{0}\left(D, \Omega_{D}^{r-1}\right)$ with $\left(\left.\gamma\right|_{\Delta}\right)^{*}(\tau)=\sigma$. This finishes the proof of Claim 11.13 
Finally, using the isomorphisms 111.12.2, 11.12.3 and Claim 11.13 established above, Sequence 11.12.1 translates into

$$
0 \rightarrow \Omega_{X / T}^{[r]} \rightarrow \Omega_{X / T}^{[r]}(\log D) \stackrel{\rho^{[r]}}{\longrightarrow} \Omega_{D / T}^{r-1} \rightarrow 0
$$

which is the sequence whose existence is asserted in 11.711. Using Fact 11.5 and using that the finite covering $\gamma$ is étale away from the singular locus of $(X, D)$, it follows by construction that the map $\rho^{[r]}$ coincides with the usual relative residue map wherever the pair $(X, D)$ is snc.

11.C.3. Proof of Theorem 11.7 construction and exactness of (11.72). Restricting the morphism $\rho^{[r]}$ of the sequence 11.13 .2 to the smooth variety $D \subset X$, and recalling that restriction is a right-exact functor, we obtain a surjection

$$
\left.\rho^{[r]}\right|_{D}:\left.\Omega_{X / T}^{[r]}(\log D)\right|_{D} \rightarrow \Omega_{D / T}^{r-1} \rightarrow 0 .
$$

Since any sheaf morphism to a reflexive sheaf factors via the reflexive hull of the domain, 11.13.3 induces a surjective map between reflexive hulls, and therefore an exact sequence

$$
\left.0 \rightarrow \operatorname{ker}\left(\rho_{D}^{[r]}\right) \rightarrow \Omega_{X / T}^{[r]}(\log D)\right|_{D} ^{* *} \stackrel{\rho_{D}^{[r]}}{\longrightarrow} \Omega_{D / T}^{r-1} \rightarrow 0 .
$$

Comparing (11.72) and 11.13.4), we see that to finish the proof of Theorem 11.7, we need to show that

$$
\operatorname{ker}\left(\rho_{D}^{[r]}\right) \simeq \Omega_{D / T}^{[r]}
$$

To this end, we consider the standard restricted residue sequence 11.2) for the morphism $\psi$, and its $G$-invariant push-forward,

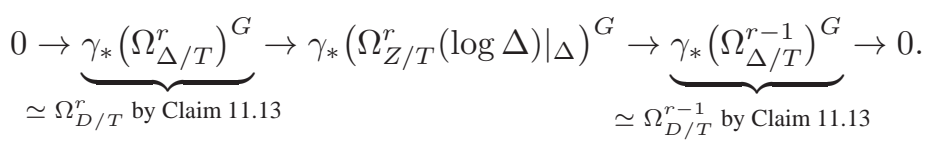

By Lemma B.3 from Appendix B, this sequence is exact. In order to describe the middle term of 11.13.5 and to relate 11.13.5 to (11.13.4), observe that the Restriction Lemma B.6 together with the isomorphism 11.12.3 yields a surjective sheaf morphism

$$
\varphi:\left.\Omega_{X / T}^{[r]}(\log D)\right|_{D} ^{* *} \rightarrow \gamma_{*}\left(\left.\Omega_{Z / T}^{r}(\log \Delta)\right|_{\Delta}\right)^{G} .
$$

Since $\gamma$ is étale in codimension one, it is étale at the general point of $\Delta$, and hence $\varphi$ is generically an isomorphism. Consequently $\varphi$ is an isomorphism as $\left.\Omega_{X / T}^{[r]}(\log D)\right|_{D} ^{* *}$ is torsion-free. Additionally, it follows from Fact 11.5 that the map $\varphi$ fits into the following commutative diagram with exact rows,

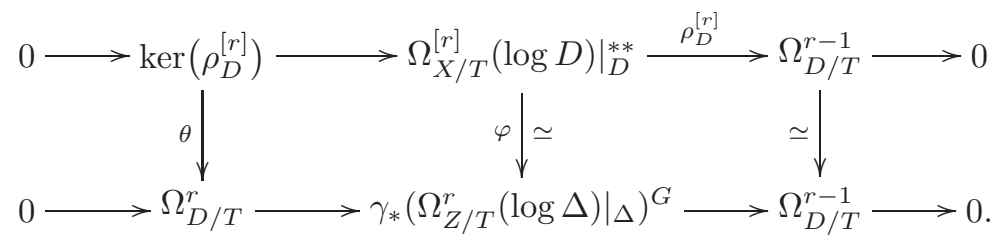

This shows that $\theta$ is an isomorphism, and completes the proof of Theorem 11.7 


\section{THE RESIDUE MAP FOR 1-FORMS}

Let $X$ be a smooth variety and $D \subset X$ a smooth, irreducible divisor. The first residue sequence 11.1 of the pair $(X, D)$ then reads

$$
\left.0 \rightarrow \Omega_{D}^{1} \rightarrow \Omega_{X}^{1}(\log D)\right|_{D} \stackrel{\rho^{1}}{\rightarrow} \mathscr{O}_{D} \rightarrow 0,
$$

and we obtain a connecting morphism of the long exact cohomology sequence,

$$
\delta: H^{0}\left(D, \mathscr{O}_{D}\right) \rightarrow H^{1}\left(D, \Omega_{D}^{1}\right)
$$

In this setting, the standard description of the first Chern class in terms of the connecting morphism, [Har77, III. Ex. 7.4], asserts that

$$
c_{1}\left(\left.\mathscr{O}_{X}(D)\right|_{D}\right)=\delta\left(\mathbf{1}_{D}\right) \in H^{1}\left(D, \Omega_{D}^{1}\right),
$$

where $\mathbf{1}_{D}$ is the constant function on $D$ with value one.

12.A. Main result of this section. Theorem 12.2 generalises the Identity 12.1 to the case where $(X, D)$ is a reduced dlt pair with irreducible boundary divisor.

Theorem 12.2. Let $(X, D)$ be a dlt pair, $D=\lfloor D\rfloor$ irreducible. Then, there exists a closed subset $Z \subset X$ with $\operatorname{codim}_{X} Z \geq 3$ and a number $m \in \mathbb{N}$ such that $m D$ is Cartier on $X^{\circ}:=X \backslash Z$, such that $D^{\circ}:=D \cap X^{\circ}$ is smooth, and such that the restricted residue sequence

$$
\left.0 \rightarrow \Omega_{D}^{1} \rightarrow \Omega_{X}^{[1]}(\log D)\right|_{D} ^{* *} \stackrel{\rho_{D}}{\longrightarrow} \mathscr{O}_{D} \rightarrow 0
$$

defined in Theorem 11.7 is exact on $D^{\circ}$. Moreover, for the connecting homomorphism $\delta$ in the associated long exact cohomology sequence

$$
\delta: H^{0}\left(D^{\circ}, \mathscr{O}_{D^{\circ}}\right) \rightarrow H^{1}\left(D^{\circ}, \Omega_{D^{\circ}}^{1}\right)
$$

we have

$$
\delta\left(m \cdot \mathbf{1}_{D^{\circ}}\right)=c_{1}\left(\left.\mathscr{O}_{X^{\circ}}\left(m D^{\circ}\right)\right|_{D^{\circ}}\right) .
$$

12.B. Proof of Theorem 12.2. Using Propositions 9.1,9.13 and Theorem 11.7 to remove from $X$ a suitable subset of codimension 3, we may assume that the following holds.

Additional Assumption 12.3. The divisor $D$ is smooth. The variety $X$ is $\mathbb{Q}$-factorial, so that there exists a number $m$ such that $m D$ is Cartier. The restricted residue sequence,

$$
\left.0 \rightarrow \Omega_{D}^{1} \stackrel{i^{1}}{\rightarrow} \Omega_{X}^{[1]}(\log D)\right|_{D} ^{* *} \stackrel{\rho_{D}}{\rightarrow} \mathscr{O}_{D} \rightarrow 0
$$

is exact.

Let $X^{\circ \circ}=(X, D)_{\text {reg }}$ be the snc locus of $(X, D)$, and set $D^{\circ \circ}=D \cap X^{\circ \circ}$.

12.B.1. Čech-cocycles describing the line bundle $\mathscr{O}_{X}(m D)$ and its Chern class. Since $m D$ is Cartier, there exists a covering of $D$ by open affine subsets $\left(U_{\alpha}\right)_{\alpha \in I}$ and there are functions $f_{\alpha} \in \mathscr{O}_{X}\left(U_{\alpha}\right)$ cutting out the divisors $\left.m D\right|_{U_{\alpha}}$, for all $\alpha \in A$.

Setting $g_{\alpha \beta}:=f_{\alpha} / f_{\beta} \in H^{0}\left(U_{\alpha} \cap U_{\beta}, \mathscr{O}_{U_{\alpha} \cap U_{\beta}}^{*}\right)$, the line bundle $\left.\mathscr{O}_{X}(m D)\right|_{D} \in$ $\operatorname{Pic}(D)=H^{1}\left(D, \mathscr{O}_{D}^{*}\right)$ is represented by the C̆ech-cocycle

$$
\left(\left.g_{\alpha \beta}\right|_{D}\right)_{\alpha, \beta} \in \breve{\mathrm{C}}^{1}\left(\left\{U_{\alpha} \cap D\right\}_{\alpha \in I}, \mathscr{O}_{D}^{*}\right) \text {. }
$$

In particular, the first Chern class $c_{1}\left(\left.\mathscr{O}_{X}(m D)\right|_{D}\right) \in H^{1}\left(D, \Omega_{D}^{1}\right)$ is represented by the Čech-cocycle

$$
\left(d \log \left(\left.g_{\alpha \beta}\right|_{D}\right)\right)_{\alpha, \beta} \in \breve{\mathrm{C}}^{1}\left(\left\{U_{\alpha} \cap D\right\}_{\alpha \in I}, \Omega_{D}^{1}\right) .
$$


12.B.2. Computation of the connecting morphism, completion of the proof. We finish the proof of Theorem 12.2 with an explicit computation of the connecting morphism. The following claim will prove to be crucial.

Claim 12.4. For any index $\alpha$, consider the Kähler differential $d \log f_{\alpha} \in$ $H^{0}\left(U_{\alpha}, \Omega_{X}^{1}(\log D)\right)$, with associated section

$$
\sigma_{\alpha} \in H^{0}\left(U_{\alpha} \cap D,\left.\Omega_{X}^{[1]}(\log D)\right|_{D} ^{* *}\right) .
$$

Then $\rho_{D}\left(\sigma_{\alpha}\right)=m \cdot \mathbf{1}_{D \cap U_{\alpha}}$.

Proof. Given an index $\alpha$, Claim 12.4 needs only to be checked on the open set $U_{\alpha} \cap D^{\circ \circ} \subseteq$ $U_{\alpha} \cap D$. There, it follows from Equation 11.4.1 of Fact 11.4.

Claim 12.5. For any indices $\alpha, \beta$, consider the Kähler differential

$$
\tau_{\alpha \beta}:=d \log \left(\left.g_{\alpha \beta}\right|_{D}\right) \in H^{0}\left(U_{\alpha} \cap U_{\beta} \cap D, \Omega_{D}^{1}\right) .
$$

Then $i^{1}\left(\tau_{\alpha \beta}\right)=\sigma_{\alpha}-\sigma_{\beta}$.

Proof. Given any two indices $\alpha, \beta$, Claim 12.5 needs only to be checked on $U_{\alpha} \cap U_{\beta} \cap D^{\circ \circ}$. There, we have

$$
\begin{aligned}
i^{1}\left(d \log \left(g_{\alpha \beta} \mid D^{\circ \circ}\right)\right) & =\frac{1}{g_{\alpha \beta} \mid D^{\circ \circ}} i^{1}\left(d\left(g_{\alpha \beta} \mid D^{\circ \circ}\right)\right)=\left.\frac{1}{g_{\alpha \beta}} d\left(g_{\alpha \beta}\right)\right|_{D^{\circ \circ}} \\
& =\left.\left(d \log g_{\alpha \beta}\right)\right|_{D^{\circ \circ}}=\left.\left(d \log f_{\alpha}-d \log f_{\beta}\right)\right|_{D^{\circ \circ}}=\left.\left(\sigma_{\alpha}-\sigma_{\beta}\right)\right|_{D^{\circ \circ}},
\end{aligned}
$$

the second equality coming from Equation 11.4.2 of Fact 11.4, proving Claim 12.5.

As an immediate consequence of Claim 12.5 we obtain that $\delta\left(m \cdot \mathbf{1}_{D}\right) \in H^{1}\left(D, \Omega_{D}^{1}\right)$ is represented by the $\breve{C e c h-c o c y c l e ~}$

$$
\tau_{\alpha \beta} \in \breve{\mathrm{C}}^{1}\left(\left\{U_{\alpha} \cap D\right\}_{\alpha \in I}, \Omega_{D}^{1}\right) .
$$

Since $\tau_{\alpha \beta}=d \log \left(\left.g_{\alpha \beta}\right|_{D}\right)$, a comparison with the Čech-cocycle that describes $c_{1}\left(\left.\mathscr{O}_{X}(m D)\right|_{D}\right)$, as given in 12.3.2), then finishes the proof of Theorem 12.2

\section{PART IV. COHOMOLOGICAL METHODS}

\section{VANISHING RESULTS FOR PAIRS OF DU BOIS SPACES}

In this section we prove a vanishing theorem for reduced pairs $(X, D)$ where both $X$ and $D$ are Du Bois. A vanishing theorem for ideal sheaves on log canonical pairs (that are not necessarily reduced) will follow. Du Bois singularities are defined via Deligne's Hodge theory. We will briefly recall Du Bois's construction of the generalised de Rham complex, which is called the Deligne-Du Bois complex. Recall, that if $X$ is a smooth complex algebraic variety of dimension $n$, then the sheaves of differential $p$-forms with the usual exterior differentiation give a resolution of the constant sheaf $\mathbb{C}_{X}$. I.e., one has a complex of sheaves,

$$
\mathscr{O}_{X} \stackrel{d}{\longrightarrow} \Omega_{X}^{1} \stackrel{d}{\longrightarrow} \Omega_{X}^{2} \stackrel{d}{\longrightarrow} \Omega_{X}^{3} \stackrel{d}{\longrightarrow} \cdots \stackrel{d}{\longrightarrow} \Omega_{X}^{n} \simeq \omega_{X}
$$

which is quasi-isomorphic to the constant sheaf $\mathbb{C}_{X}$ via the natural map $\mathbb{C}_{X} \rightarrow \mathscr{O}_{X}$ given by considering constants as holomorphic functions on $X$. Recall that this complex is not a complex of quasi-coherent sheaves. The sheaves in the complex are quasi-coherent, but the maps between them are not $\mathscr{O}_{X}$-module morphisms. Notice however that this is actually 
not a shortcoming; as $\mathbb{C}_{X}$ is not a quasi-coherent sheaf, one cannot expect a resolution of it in the category of quasi-coherent sheaves.

The Deligne-Du Bois complex is a generalisation of the de Rham complex to singular varieties. It is a filtered complex of sheaves on $X$ that is quasi-isomorphic to the constant sheaf, $\mathbb{C}_{X}$. The terms of this complex are harder to describe but its properties, especially cohomological properties are very similar to the de Rham complex of smooth varieties. In fact, for a smooth variety the Deligne-Du Bois complex is quasi-isomorphic to the de Rham complex, so it is indeed a direct generalisation.

The construction of this complex, $\underline{\Omega}_{X}^{*}$, is based on simplicial resolutions. The reader interested in the details is referred to the original article [DB81]. Note also that a simplified construction was later obtained in [Car85] and [GNPP88] via the general theory of polyhedral and cubic resolutions. An easily accessible introduction can be found in [Ste85]. Other useful references are the recent book [PS08] and the survey [KS09]. The word "hyperresolution" will refer to either a simplicial, polyhedral, or cubic resolution. Formally, the construction of $\underline{\Omega}_{X}^{\circ}$ is the same regardless the type of resolution used and no specific aspects of either types will be used. We will actually not use these resolutions here. They are needed for the construction, but if one is willing to believe the basic properties then one should be able follow the material presented here.

The bare minimum we need is that there exists a filtered complex $\underline{\Omega}_{X}^{\cdot}$ unique up to quasi-isomorphism satisfying a number of properties. As a filtered complex, it admits an associated graded complex, which we denote by $G r_{\text {filt }}^{p} \underline{\Omega}_{X}^{*}$. In order to make the formulas work the way they do in the smooth case we need to make a shift. We will actually prefer to use the following notation:

$$
\underline{\Omega}_{X}^{p}:=G r_{\text {filt }}^{p} \underline{\Omega}_{X}^{*}[p],
$$

Here " $[p]$ " means that the $m^{\text {th }}$ object of the complex $\underline{\Omega}_{X}^{p}$ is defined to be the $(m+p)^{\text {th }}$ object of the complex $G r_{\text {filt }}^{p} \underline{\Omega}_{X}$. In other words, these complexes are almost the same, only one is a shifted version of the other. They naturally live in the filtered derived category of $\mathscr{O}_{X}$-modules with differentials of order $\leq 1$. For an extensive list of their properties see [DB81] or [KS09, 4.2]. Here we will only recall a few of them.

One of the most important characteristics of the Deligne-Du Bois complex is the existence of a natural morphism in the derived category $\mathscr{O}_{X} \rightarrow \underline{\Omega}_{X}^{0}$, cf. [DB81, 4.1]. We will be interested in situations where this map is a quasi-isomorphism. If this is the case and if in addition $X$ is proper over $\mathbb{C}$, the degeneration of the Frölicher spectral sequence at $E_{1}$, cf. [DB81, 4.5] or [KS09, 4.2.4], implies that the natural map

$$
H^{i}\left(X^{\mathrm{an}}, \mathbb{C}\right) \rightarrow H^{i}\left(X, \mathscr{O}_{X}\right)=\mathbb{H}^{i}\left(X, \underline{\Omega}_{X}^{0}\right)
$$

is surjective. Here $\mathbb{H}^{i}$ stands for hypercohomology of complexes, i.e., $\mathbb{H}^{i}=R^{i} \Gamma$.

Definition 13.1. A scheme $X$ is said to have Du Bois singularities (or DB singularities for short) if the natural map $\mathscr{O}_{X} \rightarrow \underline{\Omega}_{X}^{0}$ is a quasi-isomorphism.

Example 13.2. It is easy to see that smooth points are Du Bois. Deligne proved that normal crossing singularities are Du Bois as well cf. [DBJ74, Lem. 2(b)].

We are now ready to state and prove our vanishing results for pairs of Du Bois spaces. While we will only use Corollary 13.4 in this paper, we believe that these vanishing results are interesting on their own. For instance, based on these observations one may argue that a pair of Du Bois spaces is not too far from a space with rational singularities. Indeed, if $X$ has rational singularities and $D=\emptyset$, then the result of Theorem 13.3 follows directly from the definition of rational singularities. Of course, Du Bois singularities are not necessarily 
rational and hence one cannot expect vanishing theorems for the higher direct images of the structure sheaf, but our result says that there are vanishing results for ideal sheaves of Du Bois subspaces.

Theorem 13.3 (Vanishing for ideal sheaves on pairs of Du Bois spaces). Let $(X, D)$ be a reduced pair such that $X$ and $D$ are both Du Bois, and let $\pi: \widetilde{X} \rightarrow X$ be a log resolution of $(X, D)$ with $\pi$-exceptional set $E$. If we set $\widetilde{D}:=\operatorname{supp}\left(E+\pi^{-1}(D)\right)$, then

$$
R^{i} \pi_{*} \mathscr{O}_{\widetilde{X}}(-\widetilde{D})=0 \quad \text { for all } i>\max (\operatorname{dim} \overline{\pi(E) \backslash D}, 0)
$$

In particular, if $X$ is of dimension $n \geq 2$, then $R^{n-1} \pi_{*} \mathscr{O}_{\widetilde{X}}(-\widetilde{D})=0$.

Corollary 13.4 (Vanishing for ideal sheaves on $\log$ canonical pairs). Let $(X, D)$ be a $\log$ canonical pair of dimension $n \geq 2$. Let $\pi: \widetilde{X} \rightarrow X$ be a log resolution of $(X, D)$ with $\pi$-exceptional set $E$. If we set $\widetilde{D}:=\operatorname{supp}\left(E+\pi^{-1}\lfloor D\rfloor\right)$, then

$$
R^{n-1} \pi_{*} \mathscr{O}_{\widetilde{X}}(-\widetilde{D})=0
$$

Proof. Recall from [KK10b, Theorem 1.4] that $X$ is Du Bois, and that any finite union of $\log$ canonical centres is likewise Du Bois. Since the components of $\lfloor D\rfloor$ are log canonical centres, Theorem 13.3 applies to the reduced pair $(X,\lfloor D\rfloor)$ to prove the claim. For this, recall from Lemma 2.15 that the morphism $\pi$ is a $\log$ resolution of the pair $(X,\lfloor D\rfloor)$ and therefore satisfies all the conditions listed in Theorem 13.3

13.A. Preparation for the proof of Theorem 13.3. Before we give the proof of Theorem 13.3 in Section 13.B we need the following auxiliary result. This generalises parts of [GNPP88, III.1.17].

Lemma 13.5. Let $X$ be a positive dimensional variety. Then the $i^{\text {th }}$ cohomology sheaf of $\underline{\Omega}_{X}^{0}$ vanishes for all $i \geq \operatorname{dim} X$, i.e., $h^{i}\left(\underline{\Omega}_{X}^{0}\right)=0$ for all $i \geq \operatorname{dim} X$.

Proof. For $i>\operatorname{dim} X$, the statement follows from [GNPP88, III.1.17], so we only need to prove the case when $i=n:=\operatorname{dim} X$. Let $S:=\operatorname{Sing} X$ and $\pi: \widetilde{X} \rightarrow X$ a strong $\log$ resolution with exceptional divisor $E$. Recall from [DB81, 3.2] that there are natural restriction maps, $\underline{\Omega}_{X}^{0} \rightarrow \underline{\Omega}_{S}^{0}$ and $\underline{\Omega}_{\widetilde{X}}^{0} \rightarrow \underline{\Omega}_{E}^{0}$ that reduce to the usual restriction of regular functions if the spaces are Du Bois. These maps are connected via an an exact triangle by [DB81, Prop. 4.11]:

$$
\underline{\Omega}_{X}^{0} \longrightarrow \underline{\Omega}_{S}^{0} \oplus R \pi_{*} \underline{\Omega}_{\widetilde{X}}^{0} \stackrel{\alpha}{\longrightarrow} R \pi_{*} \underline{\Omega}_{E}^{0} \stackrel{+1}{\longrightarrow}
$$

Since $\widetilde{X}$ is smooth and $E$ is an snc divisor, they are both Du Bois, cf. Example 13.2. Hence, there exist quasi-isomorphisms $\underline{\Omega}_{\widetilde{X}}^{0} \simeq \mathscr{O}_{\widetilde{X}}$ and $\underline{\Omega}_{E}^{0} \simeq \mathscr{O}_{E}$. It follows that $\alpha\left(0,{ }_{-}\right)$is the map $R \pi_{*} \mathscr{O}_{\tilde{X}} \rightarrow R \pi_{*} \mathscr{O}_{E}$ induced by the short exact sequence

$$
0 \rightarrow \mathscr{O}_{\widetilde{X}}(-E) \rightarrow \mathscr{O}_{\widetilde{X}} \rightarrow \mathscr{O}_{E} \rightarrow 0
$$

Next, consider the long exact sequence of cohomology sheaves induced by the exact triangle 13.5.1,

$\cdots \rightarrow h^{n-1}\left(\underline{\Omega}_{S}^{0}\right) \oplus R^{n-1} \pi_{*} \mathscr{O}_{\widetilde{X}} \stackrel{\alpha^{n-1}}{\rightarrow} R^{n-1} \pi_{*} \mathscr{O}_{E} \rightarrow h^{n}\left(\underline{\Omega}_{X}^{0}\right) \rightarrow h^{n}\left(\underline{\Omega}_{S}^{0}\right) \oplus R^{n} \pi_{*} \mathscr{O}_{\widetilde{X}}$.

Since $\operatorname{dim} S<n$, [GNPP88, III.1.17] implies that $h^{n}\left(\underline{\Omega}_{S}^{0}\right)=0$. Furthermore, as $\pi$ is birational, the dimension of any fibre of $\pi$ is at most $n-1$ and hence $R^{n} \pi_{*} \mathscr{O}_{\widetilde{X}}=0$. This implies that $h^{n}\left(\underline{\Omega}_{X}^{0}\right) \simeq$ coker $\alpha^{n-1}$. The bound on the dimension of the fibres of $\pi$ also implies that $R^{n} \pi_{*} \mathscr{O}_{\widetilde{X}}(-E)=0$, so taking into account the observation above about 
the map $(\alpha, 0)$, we obtain that $\alpha^{n-1}(0, \ldots)$ is surjective, and then naturally so is $\alpha^{n-1}$. Therefore, $h^{n}\left(\underline{\Omega}_{X}^{0}\right) \simeq \operatorname{coker} \alpha^{n-1}=0$.

13.B. Proof of Theorem 13.3. Since the divisor $D$ is assumed to be reduced, we simplify notation in this proof and use the symbol $D$ to denote both the divisor and its support. To start the proof, set $\Sigma:=\overline{\pi(E) \backslash D}$ and $s:=\max (\operatorname{dim} \Sigma, 0)$. Let $\Gamma:=D \cup \pi(E)$ and consider the exact triangle from [DB81, 4.11],

$$
\underline{\Omega}_{X}^{0} \longrightarrow \underline{\Omega}_{\Gamma}^{0} \oplus R \pi_{*} \underline{\Omega}_{\widetilde{X}}^{0} \longrightarrow R \pi_{*} \underline{\Omega}_{\widetilde{D}}^{0} \stackrel{+1}{\longrightarrow} .
$$

Since $\widetilde{X}$ is smooth and $\widetilde{D}$ is a snc divisor, we have quasi-isomorphisms $R \pi_{*} \underline{\Omega}_{\widetilde{X}}^{0} \simeq R \pi_{*} \mathscr{O}_{\widetilde{X}}$ and $R \pi_{*} \underline{\Omega}_{\widetilde{D}}^{0} \simeq R \pi_{*} \mathscr{O}_{\widetilde{D}}$, so this exact triangle induces the following long exact sequence of sheaves:

$$
\cdots \rightarrow h^{i}\left(\underline{\Omega}_{X}^{0}\right) \rightarrow h^{i}\left(\underline{\Omega}_{\Gamma}^{0}\right) \oplus R^{i} \pi_{*} \mathscr{O}_{\widetilde{X}} \rightarrow R^{i} \pi_{*} \mathscr{O}_{\widetilde{D}} \rightarrow h^{i+1}\left(\underline{\Omega}_{X}^{0}\right) \rightarrow \cdots
$$

By assumption $h^{i}\left(\underline{\Omega}_{X}^{0}\right)=h^{i}\left(\underline{\Omega}_{D}^{0}\right)=0$ for $i>0$. Furthermore, $h^{i}\left(\underline{\Omega}_{\Sigma}^{0}\right)=0$ and $h^{i-1}\left(\underline{\Omega}_{\Sigma \cap D}^{0}\right)=0$ for $i \geq s$ by Lemma13.5 Hence, $h^{i}\left(\underline{\Omega}_{\Gamma}^{0}\right)=0$ for $i \geq s$ by [DB81, 3.8]. As in the proof of Lemma 13.5 we obtain that the natural restriction map

$$
R^{i} \pi_{*} \mathscr{O}_{\widetilde{X}} \rightarrow R^{i} \pi_{*} \mathscr{O}_{\widetilde{D}}
$$

is surjective for $i \geq s$ and is an isomorphism for $i>s$. This in turn implies that $R^{i} \pi_{*} \mathscr{O}_{\widetilde{X}}(-\widetilde{D})=0$ for $i>s$ as desired.

\section{STEENBRINK-TYPE VANISHING RESUlts FOR LOG CANONICAL PAIRS}

The second vanishing theorem we shall need to prove the main result is concerned with direct images of logarithmic sheaves.

Theorem 14.1 (Steenbrink-type vanishing for log canonical pairs). Let $(X, D)$ be a log canonical pair of dimension $n \geq 2$. If $\pi: \widetilde{X} \rightarrow X$ is a log resolution of $(X, D)$ with $\pi$-exceptional set $E$ and $\widetilde{D}:=\operatorname{supp}\left(E+\pi^{-1}\lfloor D\rfloor\right)$, then

$$
R^{n-1} \pi_{*}\left(\Omega_{\widetilde{X}}^{p}(\log \widetilde{D}) \otimes \mathscr{O}_{X}(-\widetilde{D})\right)=0 \quad \text { for all } 0 \leq p \leq n .
$$

Remark 14.1.1. Recall from Lemma 2.15 that $\pi$ is also a log resolution of the pair $(X,\lfloor D\rfloor)$. In particular, it follows from the definition that $\widetilde{D}$ is of pure codimension one and has simple normal crossing support.

Remark 14.1.2. For $p>1$ the claim of Theorem 14.1 is proven in [Ste85, Thm. 2(b)] without any assumption on the nature of the singularities of $X$. The case $p=0$ is covered by Theorem 13.3. Hence, the crucial statement is the vanishing for $p=1$ in the case of $\log$ canonical singularities.

Corollary 14.2 (Steenbrink-type vanishing for cohomology with supports). Let $(X, D)$ be a log canonical pair of dimension $n \geq 2$. Let $\pi: \widetilde{X} \rightarrow X$ be a log resolution of $(X, D)$ with $\pi$-exceptional set $E$ and set $\widetilde{D}:=\operatorname{supp}\left(E+\pi^{-1} \operatorname{supp}\lfloor D\rfloor\right)$. If $x \in X$ is any point with set-theoretic fibre $F_{x}=\pi^{-1}(x)_{\mathrm{red}}$, then

$$
H_{F_{x}}^{1}\left(\widetilde{X}, \Omega_{\widetilde{X}}^{p}(\log \widetilde{D})\right)=0 \quad \text { for all } 0 \leq p \leq n .
$$

Remark 14.2.1. Using the standard exact sequence for cohomology with support, Har77, Ex.III.2.3(e)], the conclusion of Corollary 14.2 can equivalently be reformulated in terms of restriction maps as follows. 
(14.2.1) The map $H^{0}\left(\widetilde{X}, \Omega_{\widetilde{X}}^{p}(\log \widetilde{D})\right) \rightarrow H^{0}\left(\widetilde{X} \backslash F_{x}, \Omega_{\widetilde{X}}^{p}(\log \widetilde{D})\right)$ is surjective, and (14.2.2) the map $H^{1}\left(\widetilde{X}, \Omega_{\widetilde{X}}^{p}(\log \widetilde{D})\right) \rightarrow H^{1}\left(\widetilde{X} \backslash F_{x}, \Omega_{\widetilde{X}}^{p}(\log \widetilde{D})\right)$ is injective.

Proof of Corollary 14.2 Duality for cohomology groups with support, cf. [GKK10, Appendix], yields that

$$
H_{F_{x}}^{1}\left(\widetilde{X}, \Omega_{\widetilde{X}}^{p}(\log \widetilde{D})\right) \stackrel{\text { dual }}{\sim}\left(R^{n-1} \pi_{*} \Omega_{\widetilde{X}}^{n-p}(\log \widetilde{D})(-\widetilde{D})_{x}\right) \widehat{,}
$$

where denotes completion with respect to the maximal ideal $\mathfrak{m}_{x}$ of the point $x \in X$. The latter group vanishes by Theorem 14.1

14.A. Preparation for the proof of Theorem 14.1: Topological vanishing. To prepare for the proof of Theorem 14.1, we first discuss the local topology of the pair $(\widetilde{X}, \widetilde{D})$ near a fibre of $\pi$ and derive a topological vanishing result, which is probably well-known to experts. Subsequently, the vanishing for coherent cohomology groups claimed in Theorem 14.1 follows from an argument going back to Wahl [Wah85, §1.5].

Remark 14.3. Note that we will work in the complex topology of $X$ and $\widetilde{X}$ and we will switch back and forth between cohomology of coherent algebraic sheaves and the cohomology of their analytification without further indication. This is justified by the relative version of Serre's GAGA results, cf. [KM98, Thm. 2.48].

Lemma 14.4 (Topological vanishing). In the setup of Theorem 14.1 if $j: \widetilde{X} \backslash \widetilde{D} \hookrightarrow \widetilde{X}$ is the inclusion map, and if $j ! \mathbb{C}_{\widetilde{X} \backslash \widetilde{D}}$ is the sheaf that is defined by the short exact sequence

$$
0 \longrightarrow j ! \mathbb{C}_{\widetilde{X} \backslash \widetilde{D}} \longrightarrow \mathbb{C}_{\widetilde{X}} \stackrel{\text { restriction }}{\longrightarrow} \mathbb{C}_{\widetilde{D}} \longrightarrow 0,
$$

then $R^{k} \pi_{*}\left(j_{!} \mathbb{C}_{\widetilde{X} \backslash \widetilde{D}}\right)=0$ for all numbers $k$.

Proof. Let $F_{x}$ denote the reduced fiber of $\pi$ over a point $x \in \operatorname{Supp}[D] \cup \pi(E)$. By [Loj64. Thms. 2 and 3] we can find arbitrarily fine triangulations of $\widetilde{X}$ and $\widetilde{D}$ such that $\widetilde{D}$ is a subcomplex of the triangulation of $\widetilde{X}$ and such that $F_{x}$ is a subcomplex of the triangulation of $\widetilde{D}$. It follows that there exist arbitrarily small neighbourhoods $\widetilde{U}=\widetilde{U}\left(F_{x}\right)$ of $F_{x}$ in $\widetilde{X}$ such that the inclusions $F_{x} \hookrightarrow \widetilde{D} \cap \widetilde{U} \hookrightarrow \widetilde{U}$ are homotopy-equivalences. Since $\pi$ is proper, preimages of small open neighbourhoods of $x$ in $X$ form a neighbourhood basis of the fibre $F_{x}$. As a consequence, there exist arbitrarily small neighbourhoods $U$ of $x$ in $X$ such that the natural morphisms

$$
H^{k}\left(\pi^{-1}(U),\left.\mathbb{C}_{\widetilde{X}}\right|_{\pi^{-1}(U)}\right) \rightarrow H^{k}\left(\widetilde{D} \cap \pi^{-1}(U),\left.\mathbb{C}_{\widetilde{D}}\right|_{\widetilde{D} \cap \pi^{-1}(U)}\right)
$$

are isomorphisms for all $k$. The long exact sequence derived from 14.4.1) then implies the claimed vanishing.

14.B. Proof of Theorem 14.1. As observed in Remark14.1.2 we may assume that $p=1$. Consequently, have to prove that $R^{n-1} \pi_{*}\left(\Omega_{\widetilde{X}}^{1}(\log \widetilde{D}) \otimes \mathscr{O}_{\widetilde{X}}(-\widetilde{D})\right)=0$. A straightforward local computation shows that that the following sequence of sheaves is exact,

$$
\begin{aligned}
0 \rightarrow j ! \mathbb{C}_{\widetilde{X} \backslash \widetilde{D}} \rightarrow \mathscr{O}_{X}(-\widetilde{D}) \stackrel{d}{\rightarrow} & \Omega_{\widetilde{X}}^{1}(\log \widetilde{D}) \otimes \mathscr{O}_{\widetilde{X}}(-\widetilde{D}) \stackrel{d}{\rightarrow} \cdots \\
& \cdots \stackrel{d}{\rightarrow} \Omega_{\widetilde{X}}^{n-1}(\log \widetilde{D}) \otimes \mathscr{O}_{\widetilde{X}}(-\widetilde{D}) \stackrel{d}{\rightarrow} \Omega_{\widetilde{X}}^{n} \stackrel{d}{\rightarrow} 0,
\end{aligned}
$$

where $d$ denotes the usual exterior differential. For brevity of notation, set $\mathscr{G}_{p}:=$ $\Omega_{\widetilde{X}}^{p}(\log \widetilde{D}) \otimes \mathscr{O}_{\widetilde{X}}(-\widetilde{D})$. In particular, set $\mathscr{G}_{0}:=\mathscr{O}_{X}(-\widetilde{D})$. 
Claim 14.5. We have $R^{n-1} \pi_{*}\left(d \mathscr{G}_{0}\right)=0$ and $R^{n} \pi_{*}\left(d \mathscr{G}_{0}\right)=0$.

Proof. The following short exact sequence forms the first part of the long exact sequence (14.4.2):

$$
0 \rightarrow j ! \mathbb{C}_{\widetilde{X} \backslash \widetilde{D}} \rightarrow \mathscr{G}_{0} \stackrel{d}{\rightarrow} d \mathscr{G}_{0} \rightarrow 0
$$

Hence it follows from topological vanishing, Lemma 14.4 that $R^{n-1} \pi_{*}\left(d \mathscr{G}_{0}\right) \simeq$ $R^{n-1} \pi_{*} \mathscr{G}_{0}$ and $R^{n} \pi_{*}\left(d \mathscr{G}_{0}\right) \simeq R^{n} \pi_{*} \mathscr{G}_{0}$. While $R^{n} \pi_{*} \mathscr{G}_{0}$ vanishes for dimensional reasons, the vanishing of $R^{n-1} \pi_{*} \mathscr{G}_{0}$ follows from Theorem 13.3. This finishes the proof of Claim 14.5.

Claim 14.6. The differential $d$ induces an isomorphism $R^{n-1} \pi_{*} \mathscr{G}_{1} \simeq R^{n-1} \pi_{*}\left(d \mathscr{G}_{1}\right)$.

Proof. The second short exact sequence derived from 14.4.2],

$$
0 \rightarrow d \mathscr{G}_{0} \rightarrow \mathscr{G}_{1} \stackrel{d}{\rightarrow} d \mathscr{G}_{1} \rightarrow 0,
$$

induces the following long exact sequence of higher push-forward sheaves,

$$
\cdots \rightarrow \underbrace{R^{n-1} \pi_{*}\left(d \mathscr{G}_{0}\right)}_{=0 \text { by Claim }[14.5]} \rightarrow R^{n-1} \pi_{*} \mathscr{G}_{1} \stackrel{d}{\rightarrow} R^{n-1} \pi_{*}\left(d \mathscr{G}_{1}\right) \rightarrow \underbrace{R^{n} \pi_{*}\left(d \mathscr{G}_{0}\right)}_{=0 \text { by Claim }[14.5} \rightarrow \cdots .
$$

Claim 14.6 then follows.

As a consequence of Claim 14.6 in order to prove Theorem 14.1, it suffices to show that $R^{n-1} \pi_{*}\left(d \mathscr{G}_{1}\right)=0$. This certainly follows from the following claim.

Claim 14.7. $R^{n-p} \pi_{*}\left(d \mathscr{G}_{p}\right)=0$ for all $1 \leq p \leq n$.

We prove Claim 14.7 by descending induction on $p$. For $p=n$, the claim is true since $R^{0} \pi_{*}\left(d \mathscr{G}_{n}\right)$ is isomorphic to the push-forward of the zero sheaf, and hence equals the zero sheaf. In general, assume that Claim 14.7 has been shown for all numbers that are larger than $p$, and consider the short exact sequence

$$
0 \rightarrow d \mathscr{G}_{p} \rightarrow \mathscr{G}_{p+1} \rightarrow d \mathscr{G}_{p+1} \rightarrow 0
$$

derived from (14.4.2). This yields a long exact sequence

$$
\cdots \rightarrow R^{n-(p+1)} \pi_{*}\left(d \mathscr{G}_{p+1}\right) \rightarrow R^{n-p} \pi_{*}\left(d \mathscr{G}_{p}\right) \rightarrow R^{n-p} \pi_{*} \mathscr{G}_{p+1} \rightarrow \cdots .
$$

Observe that the first group in (14.7.1) vanishes by induction, and that the last group vanishes by Steenbrink vanishing [Ste85, Thm. 2(b)]. This proves the claim and concludes the proof of Theorem 14.1

Remark 14.7.2. Greuel proves a similar result for isolated complete intersection singularities in [Gre80].

\section{GENERIC BASE CHANGE FOR COHOMOLOGY WITH SUPPORTS}

In this section we provide another technical tool for the proof of the main results: we give a local-to-global statement for cohomology groups with support in a family of normal varieties.

Theorem 15.1 (Generic base change for cohomology with supports). Let $\phi: X \rightarrow S$ be a surjective morphism with connected fibres between normal, irreducible varieties, and let $E \subset X$ be an algebraic subset such that the restriction $\left.\phi\right|_{E}$ is proper. Further, let $\mathscr{F}$ be a locally free sheaf on $X$ such that

$$
H_{E_{s}}^{1}\left(X_{s},\left.\mathscr{F}\right|_{X_{s}}\right)=0 \text { for all } s \in S,
$$




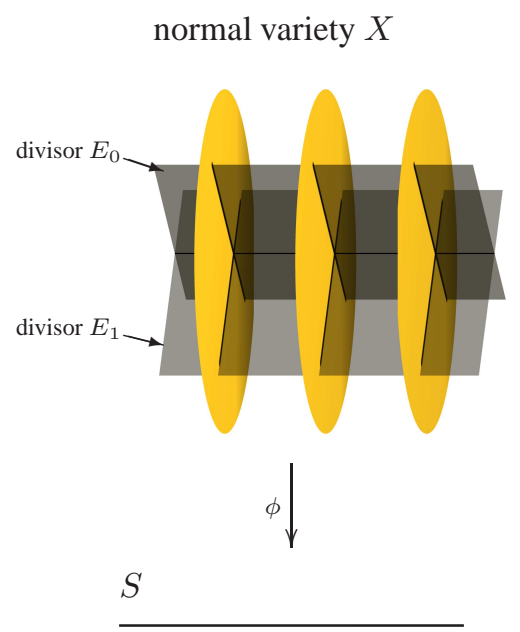

normal variety $X$

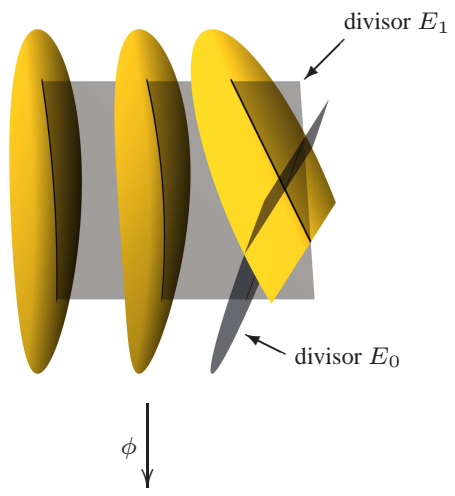

$S$

FIGURE 2. Two morphisms for which the assumptions of Theorem 15.1 hold

where $X_{s}:=\phi^{-1}(s)$ and $E_{s}:=\left(\left.\phi\right|_{E}\right)^{-1}(s)$. Then there exists a non-empty Zariski-open subset $S^{\circ} \subseteq S$, with preimage $X^{\circ}:=\phi^{-1}\left(S^{\circ}\right)$, such that

$$
H_{E \cap X^{\circ}}^{1}\left(X^{\circ},\left.\mathscr{F}\right|_{X^{\circ}}\right)=0
$$

Figure 2 illustrates the setup of Theorem 15.1 We prove Theorem 15.1 in the remainder of the present Section 15

15.A. Proof of Theorem 15.1: simplifications. To start, choose a normal, relative compactification $\bar{X}$ of $X$, i.e., a normal variety $\bar{X}$ that contains $X$ and a morphism $\Phi: \bar{X} \rightarrow S$, such that $\Phi$ is proper and $\left.\Phi\right|_{X}=\phi$. By [Gro60, I. Thm. 9.4.7] there exists a coherent extension $\overline{\mathscr{F}}$ of $\mathscr{F}$, i.e., a coherent sheaf $\overline{\mathscr{F}}$ of $\mathscr{O}_{\bar{X}}$-modules such that $\left.\overline{\mathscr{F}}\right|_{X}=\mathscr{F}$. Then excision for cohomology with supports [Har77, III Ex. 2.3(f)] asserts that the cohomology groups of (15.1.1) and (15.1.2) can be computed on $\bar{X}$. More precisely, if $S^{\circ} \subseteq S$ is a subset with preimages $X^{\circ}:=\phi^{-1}\left(S^{\circ}\right)$ and $\bar{X}^{\circ}:=\Phi^{-1}\left(S^{\circ}\right)$, then it follows from the relative properness of $E$ that

$$
H_{E \cap X^{\circ}}^{1}\left(X^{\circ}, \mathscr{F}\right) \simeq H_{E \cap \bar{X}^{\circ}}^{1}\left(\bar{X}^{\circ}, \overline{\mathscr{F}}\right) .
$$

As a consequence, we see that it suffices to show Theorem 15.1 under the following additional assumptions.

Additional Assumption 15.2. The morphism $\phi$ is proper. In particular, the higher direct image sheaves $R^{i} \phi_{*} \mathscr{F}$ are coherent sheaves of $\mathscr{O}_{S}$-modules for all $i$.

Let $\mathscr{F}_{S}:=\left.\mathscr{F}\right|_{X_{s}}$. Using semicontinuity we can replace $S$ by a suitable subset and assume without loss of generality to be in the following situation.

Additional Assumption 15.3. The variety $S$ is affine, the morphism $\phi$ is flat and the a priori upper-semicontinuous functions $s \mapsto h^{i}\left(X_{s}, \mathscr{F}_{s}\right)$ are constant for all $i$. In particular, the higher direct image sheaves $R^{i} \phi_{*} \mathscr{F}$ are all locally free.

The following excerpt from the standard cohomology sequence for cohomology with support [Har77, III Ex. 2.3(e)]

$$
H^{0}(X, \mathscr{F}) \stackrel{\alpha}{\rightarrow} H^{0}(X \backslash E, \mathscr{F}) \rightarrow H_{E}^{1}(X, \mathscr{F}) \rightarrow H^{1}(X, \mathscr{F}) \stackrel{\beta}{\rightarrow} H^{1}(X \backslash E, \mathscr{F}),
$$


shows that to prove the claim of Theorem 15.1 it is equivalent to show that $\alpha$ is surjective and that $\beta$ is injective. This is what we do next.

15.B. Proof of Theorem 15.1: surjectivity of $\boldsymbol{\alpha}$. To show surjectivity of $\alpha$, let $\sigma \in$ $H^{0}(X \backslash E, \mathscr{F})$ be any element. We need to show that there exists an element $\bar{\sigma} \in$ $H^{0}(X, \mathscr{F})$ such that $\left.\bar{\sigma}\right|_{X \backslash E}=\sigma$.

Decompose $E=E_{\text {div }} \cup E_{\text {small }}$, where $E_{\text {div }}$ has pure codimension one in $X$, and $\operatorname{codim}_{X} E_{\text {small }} \geq 2$. Since $\mathscr{F}$ is locally free in a neighbourhood of $E$, it follows immediately from the normality of $X$ that there exists a section $\sigma^{\prime} \in H^{0}\left(X \backslash E_{\mathrm{div}}, \mathscr{F}\right)$ such that $\left.\sigma^{\prime}\right|_{X \backslash E}=\sigma$. In other words, we may assume that the following holds.

Additional Assumption 15.4. The algebraic set $E$ has pure codimension one in $X$.

Since $\sigma$ is algebraic, it is clear that there exists an extension of $\sigma$ as a rational section. In other words, there exists a minimal number $k \in \mathbb{N}$ and a section

$$
\tau \in H^{0}\left(X, \mathscr{F} \otimes \mathscr{O}_{X}(k E)\right)
$$

with $\left.\tau\right|_{X \backslash E}=\sigma$. To prove surjectivity of $\alpha$, it is then sufficient to show that $k=0$. Now, if $s \in S$ is any point, it follows from the assumption made in 15.1.1) of Theorem 15.1 that there exists a section $\bar{\sigma}_{s} \in H^{0}\left(X_{s}, \mathscr{F}_{s}\right)$ such that $\left.\bar{\sigma}_{s}\right|_{X_{s} \backslash E}=\left.\sigma\right|_{X_{s} \backslash E}=\left.\tau\right|_{X_{s} \backslash E}$. Since $\mathscr{F}$ is locally free near $E$, this immediately implies that $k=0$ and that $\sigma$ is in the image of $\alpha$, as claimed.

15.C. Proof of Theorem 15.1: injectivity of $\beta$. Concerning the injectivity of $\beta$, we consider the following commutative diagram of restrictions

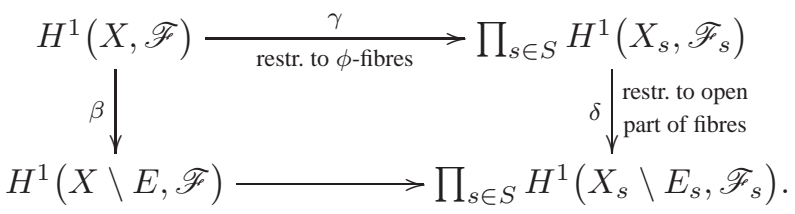

To prove injectivity of $\beta$, it is then sufficient to prove injectivity of $\gamma$ and $\delta$.

15.C.1. Injectivity of $\gamma$. Since $S$ is affine by Assumption 15.3, we have that $H^{p}\left(S, R^{q} \phi_{*} \mathscr{F}\right)=0$ for all $p>0$ and all $q$. The Leray spectral sequence, God73, II. Thm. 4.17.1], thus gives a canonical identification

$$
H^{1}(X, \mathscr{F}) \stackrel{\simeq}{\rightarrow} H^{0}\left(S, R^{1} \phi_{*} \mathscr{F}\right) .
$$

By the second part of Assumption 15.3 we may apply Grauert's Theorem [Har77, III Cor. 12.9] to obtain that the natural map

$$
R^{1} \phi_{*} \mathscr{F} \otimes \mathbb{C}(s) \stackrel{\simeq}{\longrightarrow} H^{1}\left(X_{s}, \mathscr{F}_{s}\right)
$$

is an isomorphism for any point $s \in S$. Hence the map $\gamma$ may be identified with the evaluation map,

$$
H^{0}\left(S, R^{1} \phi_{*} \mathscr{F}\right) \rightarrow \prod_{s \in S} R^{1} \phi_{*} \mathscr{F} \otimes \kappa(s),
$$

that maps a section of the locally free sheaf $R^{1} \phi_{*} \mathscr{F}$ to its values at the points of $S$. This map is clearly injective. 
15.C.2. Injectivity of $\delta$. The injectivity of $\delta$ follows immediately from the assumption made in 15.1.1) of Theorem 15.1 and from the cohomology sequence for cohomology with support, [Har77, III Ex. 2.3(e)], already discussed above. This shows injectivity of $\beta$ and completes the proof of Theorem 15.1

\section{PART V. EXTENSION WITH LOGARITHMIC POLES}

\section{MAin RESUlt OF THIS PART}

In the present Part $\square$ of this paper, we make an important step towards a full proof of the main Extension Theorem 1.5 by proving the following, weaker version of Theorem 1.5

Theorem 16.1 (Extension theorem for differential forms on log canonical pairs). Let $(X, D)$ be a $\log$ canonical pair of dimension $\operatorname{dim} X \geq 2$. Let $\pi: \widetilde{X} \rightarrow X$ be a log resolution of $(X, D)$ with exceptional set $E \subset \widetilde{X}$, and consider the reduced divisor

$$
\widetilde{D}^{\prime}:=\operatorname{supp}\left(E+\pi^{-1}\lfloor D\rfloor\right) .
$$

Then the sheaf $\pi_{*} \Omega_{\widetilde{X}}^{p}\left(\log \widetilde{D}^{\prime}\right)$ is reflexive for any number $0 \leq p \leq n$.

Theorem 1.5 and Theorem 16.1 differ only in the choice of the divisors $\widetilde{D}$ and $\widetilde{D}^{\prime}$, respectively. Theorem 16.1 is weaker than Theorem 1.5 because $\widetilde{D}^{\prime}$ is larger than $\widetilde{D}$, so that Theorem 16.1 allows the extended differential forms to have poles along a larger number of exceptional divisors then Theorem 1.5 would allow.

16.A. Reformulation of Theorem 16.1. In Part VI of this paper, Theorem 16.1 will be used to give a proof of the main Extension Theorem 1.5, and the formulation of Theorem 16.1 is designed to make this application as simple as possible. The formulation is, however, not optimal for proof. Rather than proving Theorem 16.1 directly, we have therefore found it easier to prove the following equivalent reformulation which is more suitable for inductive arguments.

Theorem 16.2 (Reformulation of Theorem 16.1). Let $(X, D)$ be a log canonical pair and let $\pi: \widetilde{X} \rightarrow X$ be a log resolution with exceptional set $E=\operatorname{Exc}(\pi)$. Consider the reduced divisor

$$
\widetilde{D}^{\prime}:=\operatorname{supp}\left(E+\pi^{-1} \operatorname{supp}\lfloor D\rfloor\right) .
$$

If $p$ is any index and $E_{0} \subseteq E$ any irreducible component, then the injective restriction map

$$
r: H^{0}\left(\widetilde{X} \backslash \operatorname{supp}\left(E-E_{0}\right), \Omega_{\widetilde{X}}^{p}\left(\log \widetilde{D}^{\prime}\right)\right) \rightarrow H^{0}\left(\widetilde{X} \backslash E, \Omega_{\widetilde{X}}^{p}\left(\log \widetilde{D}^{\prime}\right)\right)
$$

is in fact an isomorphism.

Explanation 16.3. We aim to show that Theorem 16.2 implies Theorem 16.1 To prove Theorem 16.1 we need to show that for any open set $U \subseteq X$ with preimage $\widetilde{U} \subseteq \widetilde{X}$, the natural restriction map

$$
r_{U}: H^{0}\left(\widetilde{U}, \Omega_{\widetilde{X}}^{p}\left(\log \widetilde{D}^{\prime}\right)\right) \rightarrow H^{0}\left(\widetilde{U} \backslash E, \Omega_{\widetilde{X}}^{p}\left(\log \widetilde{D}^{\prime}\right)\right)
$$

is in fact surjective. Thus, let $U \subseteq X$ be any open set, and let $\sigma \in H^{0}\left(\widetilde{U} \backslash E, \Omega_{\widetilde{X}}^{p}\left(\log \widetilde{D}^{\prime}\right)\right)$ be any form.

Assuming that Theorem 16.2 holds, it can be applied to the lc pair $(U, D)$ and to its log resolution $\left.\pi\right|_{\widetilde{U}}: \widetilde{U} \rightarrow U$. A repeated application of 16.2.1) shows that $\sigma$ extends over every single component of $E \cap \widetilde{U}$, and therefore over all of $E \cap \widetilde{U}$. Surjectivity of the map $r_{U}$ then follows, and Theorem 16.1 is shown. 


\section{Proof of Theorem 16.2}

The proof of Theorem 16.2 will be presented in this section. We will maintain the assumptions and the notation of (16.2). Since the proof is long, we chose to present it as a sequence of clearly marked and relatively independent steps.

17.A. Setup of notation and of the main induction loop. An elementary computation, explained in all detail in [GKK10, Lem. 2.13], shows that to prove Theorem 16.2 for all $\log$ resolutions of a given pair, it suffices to prove the result for one log resolution only. We may therefore assume the following without loss of generality.

Additional Assumption 17.1. The $\log$ resolution morphism $\pi$ is a strong $\log$ resolution.

The proof of Theorem 16.2 involves two nested induction loops. The main, outer loop considers pairs of numbers $\left(\operatorname{dim} X, \operatorname{codim} \pi\left(E_{0}\right)\right)$, which we order lexicographically as indicated in Table 1

\begin{tabular}{lccccccccccc} 
No. & 1 & 2 & 3 & 4 & 5 & 6 & 7 & 8 & 9 & 10 & $\cdots$ \\
\hline $\operatorname{dim} X$ & 2 & 3 & 3 & 4 & 4 & 4 & 5 & 5 & 5 & 5 & $\cdots$ \\
$\operatorname{codim} \pi\left(E_{0}\right)$ & 2 & 2 & 3 & 2 & 3 & 4 & 2 & 3 & 4 & 5 & $\cdots$
\end{tabular}

TABLE 1. Lexicographical ordering of dimensions and codimensions

17.B. Main induction loop: start of induction. The first column of Table 1 describes the case where $\operatorname{dim} X=2$ and $\operatorname{codim}_{X} \pi\left(E_{0}\right)=2$. After some reductions, it will turn out that this case has essentially been treated previously, in [GKK10]. Given a surface pair $(X, D)$ as in Theorem 16.2, consider the open subsets

$$
X^{0}:=X \backslash \operatorname{supp}(D) \text { and } X^{1}:=(X, D)_{\text {reg }} \cup \operatorname{supp}(D) \text {. }
$$

Observe that $X^{1}$ is open and that the complement of $(X, D)_{\text {reg }}$ is finite. For $i \in\{0,1\}$, we also consider the preimages $\widetilde{X}^{i}:=\pi^{-1}\left(X^{i}\right)$ and induced $\log$ resolution $\pi^{i}: \widetilde{X}^{i} \rightarrow X^{i}$. Since the statement of Theorem 16.2 is local on $X$, and since $X=X^{0} \cup X^{1}$ it suffices to prove Theorem 16.2 for the two pairs $\left(X^{0}, \emptyset\right)$ and $\left(X^{1}, D\right)$ independently.

17.B.1. Resolutions of the pair $\left(X^{0}, \emptyset\right)$. Since $X$ is a surface, the index $p$ is either zero, one or two. The case where $p=0$ is trivial. Since $\left(X^{0}, \emptyset\right)$ is reduced and log canonical, the two remaining cases are covered by earlier results. For $p=1$, Theorem 16.2 is shown in [GKK10, Prop. 7.1]. The case where $p=2$ is covered by [GKK10, Prop. 5.1].

17.B.2. Resolutions of the pair $\left(X^{1}, D\right)$. Again, we aim to apply the results of [GKK10], this time employing ideas from the discussion of boundary-lc pairs, [GKK10, Sect 3.2], for the reduction to known cases.

In complete analogy to the argument of the previous Section 17.B.1 Theorem 16.2 follows if we can apply [GKK10, Prop. 5.1 and Prop. 7.1] to the reduced pair $\left(X^{1},\lfloor D\rfloor\right)$. For that, it suffices to show that the pair $\left(X^{1},\lfloor D\rfloor\right)$ is $\log$ canonical. This follows trivially from the monotonicity of discrepancies, [KM98, Lem. 2.27], once we show that the variety $X^{1}$ is $\mathbb{Q}$-factorial.

To this end, observe that for any sufficiently small rational number $\varepsilon>0$, the nonreduced pair $\left(X^{1},(1-\varepsilon) D\right)$ is numerically dlt; see [KM98, Notation 4.1] for the definition and use [KM98, Lem. 3.41] for an explicit discrepancy computation. By [KM98, Prop. 4.11], the space $X^{1}$ is then $\mathbb{Q}$-factorial, as required. 
17.C. Main induction loop: proof of the inductive step. We are now in a setting where $\operatorname{dim} X \geq 3$. We assume that a number $p \leq \operatorname{dim} X$ and an irreducible component $E_{0} \subseteq E$ are given.

Notation 17.2. If $E$ is reducible, we denote the irreducible components of $E$ by $E_{0}, \ldots, E_{N}$, numbered in a way such that $\operatorname{dim} \pi\left(E_{1}\right) \leq \operatorname{dim} \pi\left(E_{2}\right) \leq \cdots \leq \operatorname{dim} \pi\left(E_{N}\right)$. In particular, if $E$ is reducible, then there exists a number $k \geq 0$ so that

$$
\operatorname{dim} \pi\left(E_{i}\right)>\operatorname{dim} \pi\left(E_{0}\right) \Leftrightarrow N \geq i>k .
$$

If $E$ is irreducible, we use the following obvious notational convention.

Convention 17.3. If $E$ is irreducible, set $k:=N:=0$, and write

$$
\begin{aligned}
& E_{1} \cup \cdots \cup E_{k}:=E_{1} \cup \cdots \cup E_{N}:=\emptyset, \quad \text { and } \\
& E_{0} \cup \cdots \cup E_{k}:=E_{0} \cup \cdots \cup E_{N}:=E_{0} .
\end{aligned}
$$

Convention 17.3 admittedly abuses notation. However, it has the advantage that we can give uniform formulas that work both in the irreducible and the reducible case. For instance, the restriction morphism 16.2.11 of Theorem 16.2 can now be written as

$r: H^{0}\left(\widetilde{X} \backslash\left(E_{1} \cup \cdots \cup E_{N}\right), \Omega_{\widetilde{X}}^{p}\left(\log \widetilde{D}^{\prime}\right)\right) \rightarrow H^{0}\left(\widetilde{X} \backslash\left(E_{0} \cup \cdots \cup E_{N}\right), \Omega_{\widetilde{X}}^{p}\left(\log \widetilde{D}^{\prime}\right)\right)$.

17.C.1. Main induction loop: induction hypothesis. The induction hypothesis asserts that Theorem 16.2 holds for all $\log$ resolutions of $\log$ canonical pairs $(\bar{X}, \bar{D})$ with $\operatorname{dim} \bar{X}<$ $\operatorname{dim} X$, and if $\operatorname{dim} \bar{X}=\operatorname{dim} X$, then 16.2 .1 holds for all divisors $E_{i} \subseteq E \subset \widetilde{X}$ with $\operatorname{dim} \pi\left(E_{i}\right)>\operatorname{dim} \pi\left(E_{0}\right)$.

Using Convention 17.3 and Formula 17.2.1 of Notation 17.2, the second part of the induction hypothesis implies that the horizontal arrows in following commutative diagram of restriction morphisms are both isomorphic,

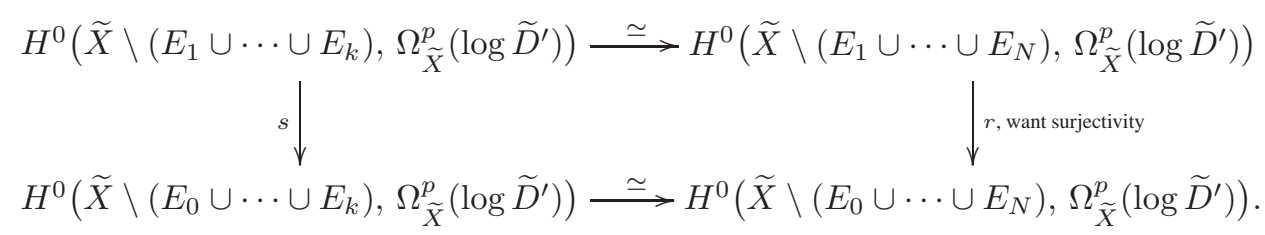

In particular, we obtain the following reformulation of the problem.

Claim 17.4. To prove Theorem 16.2 and to show surjectivity of 16.2.1), it suffices to show that the natural restriction map $s$ is surjective.

17.C.2. Simplifications. To show surjectivity of $s$ and to prove Theorem 16.2, it suffices to consider a Zariski-open subset of $X$ that intersects $\pi\left(E_{0}\right)$ non-trivially. This will allow us to simplify the setup substantially, here and in Section 17.C.4 below.

Claim 17.5. Let $X^{\circ} \subseteq X$ be any open set that intersects $\pi\left(E_{0}\right)$ non-trivially, and let $\widetilde{X}^{\circ}:=\pi^{-1}\left(X^{\circ}\right)$ be its preimage. If the restriction map

$s^{\circ}: H^{0}\left(\widetilde{X}^{\circ} \backslash\left(E_{1} \cup \cdots \cup E_{k}\right), \Omega_{\widetilde{X}}^{p}\left(\log \widetilde{D}^{\prime}\right)\right) \rightarrow H^{0}\left(\widetilde{X}^{\circ} \backslash\left(E_{0} \cup \cdots \cup E_{k}\right), \Omega_{\widetilde{X}}^{p}\left(\log \widetilde{D}^{\prime}\right)\right)$ is surjective, then the map $s$ is surjective and Theorem 16.1 holds.

Proof. Given an open set $X^{\circ}$ and assuming that the associated restriction map $s^{\circ}$ is surjective, we need to show surjectivity of $s$. As in Explanation 16.3, let

$$
\sigma \in H^{0}\left(\widetilde{X} \backslash\left(E_{0} \cup \cdots \cup E_{k}\right), \Omega_{\widetilde{X}}^{p}\left(\log \widetilde{D}^{\prime}\right)\right)
$$




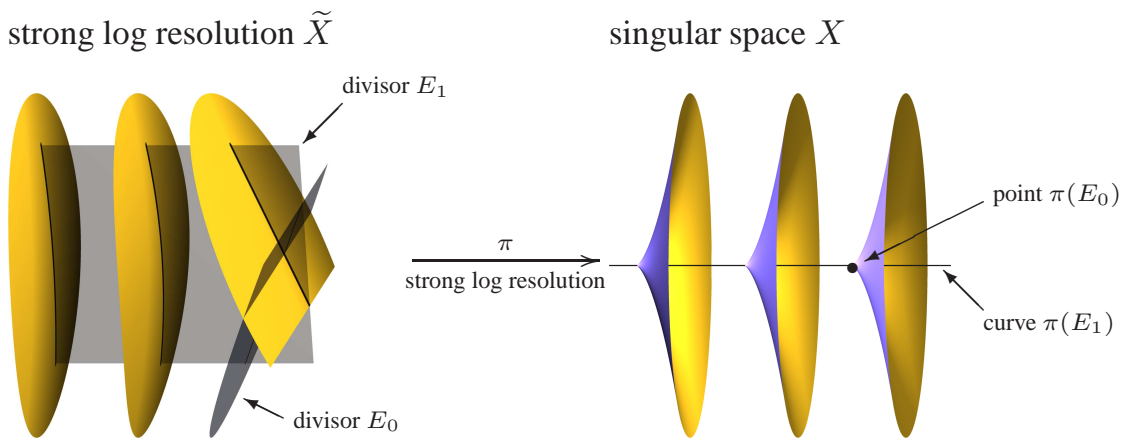

The figure sketches a situation where Assumption 17.8 holds. Here, $X$ is a threefold whose singular locus is a curve. The exceptional set of the strong $\log$ resolution $\pi$ contains two divisors $E_{0}$ and $E_{1}$. Assumption 17.8 is satisfied because $E_{0}$ is mapped to a point that is contained in the image of $E_{1}$. Another example where $\pi\left(E_{0}\right)=\pi\left(E_{1}\right)$ is shown in Figure 4 on page 49

FIGURE 3. A three-dimensional example where Assumption 17.8 holds

be any form defined away from $E_{0} \cup \cdots \cup E_{k}$, and let $c \in \mathbb{N}$ be the minimal number such that $\sigma$ extends to a section

$$
\widetilde{\sigma} \in H^{0}\left(\widetilde{X} \backslash\left(E_{1} \cup \cdots \cup E_{k}\right), \mathscr{O}_{\widetilde{X}}\left(c E_{0}\right) \otimes \Omega_{\widetilde{X}}^{p}\left(\log \widetilde{D}^{\prime}\right)\right) .
$$

We need to show that $c=0$. However, it follows from the surjectivity of 16.2.1) on $\widetilde{X}^{\circ}$ that

$$
\left.\widetilde{\sigma}\right|_{\widetilde{X}^{\circ} \backslash\left(E_{1} \cup \cdots \cup E_{k}\right)} \in H^{0}\left(\widetilde{X}^{\circ} \backslash\left(E_{1} \cup \cdots \cup E_{k}\right), \Omega_{\widetilde{X}}^{p}\left(\log \widetilde{D}^{\prime}\right)\right) .
$$

Since $\left(\widetilde{X}^{\circ} \backslash\left(E_{1} \cup \cdots \cup E_{k}\right)\right) \cap E_{0} \neq \emptyset$, this shows the claim.

17.6. We will use Claim 17.5 to simplify the situtation by replacing $X$ with appropriate open subsets successively.

Additional Assumption 17.7. The variety $X$ is affine.

Claim 17.5 also allows to remove from $X$ all images $\pi\left(E_{i}\right)$ of exceptional divisors $E_{i} \subseteq E$ with $\pi\left(E_{0}\right) \nsubseteq \pi\left(E_{i}\right)$, bringing us to the situation sketched in Figure 3. This will again simplify notation substantially.

Additional Assumption 17.8. If $E_{i} \subseteq E$ is an irreducible component, then $\pi\left(E_{0}\right) \subseteq \pi\left(E_{i}\right)$.

Observation 17.9. We have $\pi\left(E_{0}\right)=\cdots=\pi\left(E_{k}\right), E_{0} \cup \cdots \cup E_{k} \subseteq \pi^{-1}\left(\pi\left(E_{0}\right)\right)$, and

$$
\operatorname{codim}_{\tilde{X}} \pi^{-1}\left(\pi\left(E_{0}\right)\right) \backslash\left(E_{0} \cup \cdots \cup E_{k}\right) \geq 2 .
$$

Assumption 17.8 has further consequences. Because of the inequality 17.9.1), and because $\Omega_{\widetilde{X}}^{p}\left(\log \widetilde{D}^{\prime}\right)$ is a locally free sheaf on $\widetilde{X}$, any $p$-form defined on $\widetilde{X} \backslash \pi^{-1}\left(\pi\left(E_{0}\right)\right)$ will immediately extend to a $p$-form on $\widetilde{X} \backslash\left(E_{0} \cup \cdots \cup E_{k}\right)$. It follows that the bottom 
arrow in the following commutative diagram of restriction maps is in fact an isomorphism,

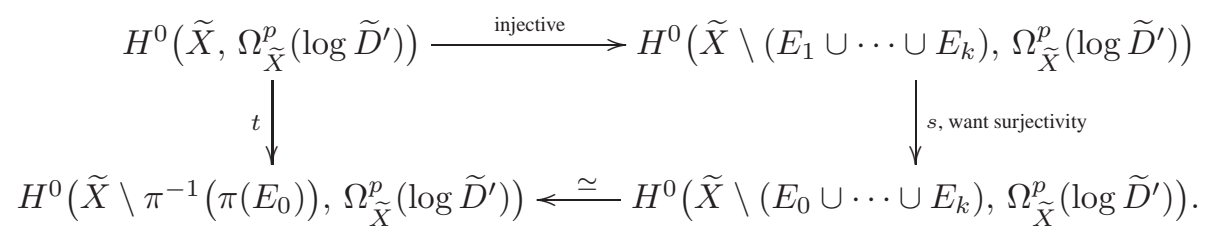

Maintaining Assumptions 17.7 and 17.8 the following is thus immediate.

Observation 17.10. To show surjectivity of $s$ and to prove Theorem 16.2, it suffices to show that the natural restriction map $t$ is surjective.

17.C.3. The case $\operatorname{dim} \pi\left(E_{0}\right)=0$. If the divisor $E_{0}$ is mapped to a point, Steenbrinktype vanishing for cohomology with supports, Corollary 14.2 applies. More precisely, the surjectivity statement 14.211 of Remark 14.2.1 asserts that the restriction morphism $t$ is surjective. This will finish the proof in case where $\operatorname{dim} \pi\left(E_{0}\right)=0$. We can therefore assume from now on that $E_{0}$ is not mapped to a point.

Additional Assumption 17.11. The variety $\pi\left(E_{0}\right)$ is smooth and has positive dimension.

17.C.4. Projection to $\pi\left(E_{0}\right)$. Given a base change diagram

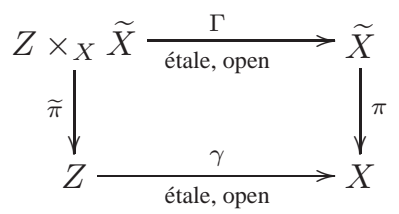

such that $\gamma^{-1}\left(\pi\left(E_{0}\right)\right) \neq \emptyset$, surjectivity of the restriction map $t$ will follow as soon as we prove surjectivity of the analogously defined map

$$
H^{0}\left(\widetilde{Z}, \Omega_{\widetilde{Z}}^{p}\left(\log \widetilde{\Delta}^{\prime}\right)\right) \rightarrow H^{0}\left(\widetilde{Z} \backslash \pi^{-1}\left(\pi\left(F_{0}\right)\right), \Omega_{\widetilde{Z}}^{p}\left(\log \widetilde{\Delta}^{\prime}\right)\right)
$$

where $\widetilde{Z}:=Z \times_{X} \widetilde{X}, \widetilde{\Delta}^{\prime}=\Gamma^{-1}\left(\widetilde{D}^{\prime}\right)$, and $F_{0}$ is a component of $\Gamma^{-1}\left(E_{0}\right)$. Since $X$ is affine by Assumption 17.7 one such diagram is given by Proposition 2.25 when projecting to the affine subvariety $\pi\left(E_{0}\right) \subset X$. Observing that $\left(Z, \gamma^{*}(D)\right)$ is lc with log-resolution $\tilde{\pi}$, that

$$
\widetilde{\Delta}^{\prime}:=\operatorname{supp}\left((\widetilde{\pi} \text {-exceptional set })+\widetilde{\pi}^{-1}\left(\operatorname{supp}\left\lfloor\gamma^{*} D\right\rfloor\right)\right),
$$

and that all additional assumptions made so far will also hold for $\widetilde{\pi}: \widetilde{Z} \rightarrow Z$, we may replace $X$ by $Z$ and assume the following without loss of generality.

Additional Assumption 17.12. There exists a smooth affine variety $T$ with a free sheaf of differentials, $\Omega_{T}^{1} \simeq \mathscr{O}_{T}^{\oplus \operatorname{dim} T}$, and a commutative diagram of surjective morphisms

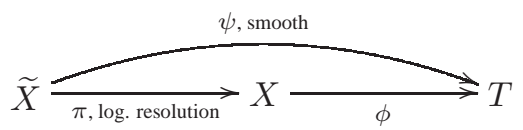

where the restriction $\left.\phi\right|_{\pi\left(E_{0}\right)}: \pi\left(E_{0}\right) \rightarrow T$ is an isomorphism and both $\phi$ and $\psi$ have connected fibres.

Additional Assumption 17.13. The composition $\psi:=\phi \circ \pi$ is an snc morphism of the pair $\left(\widetilde{X}, \widetilde{D}^{\prime}\right)$, in the sense of Definition 2.9 In particular, recall from Remark 2.10 that if $t \in T$ is any point, then the scheme-theoretic intersection $\widetilde{D}^{\prime} \cap \psi^{-1}(t)$ is reduced, of pure codimension one in $\psi^{-1}(t)$, and has simple normal crossing support. 


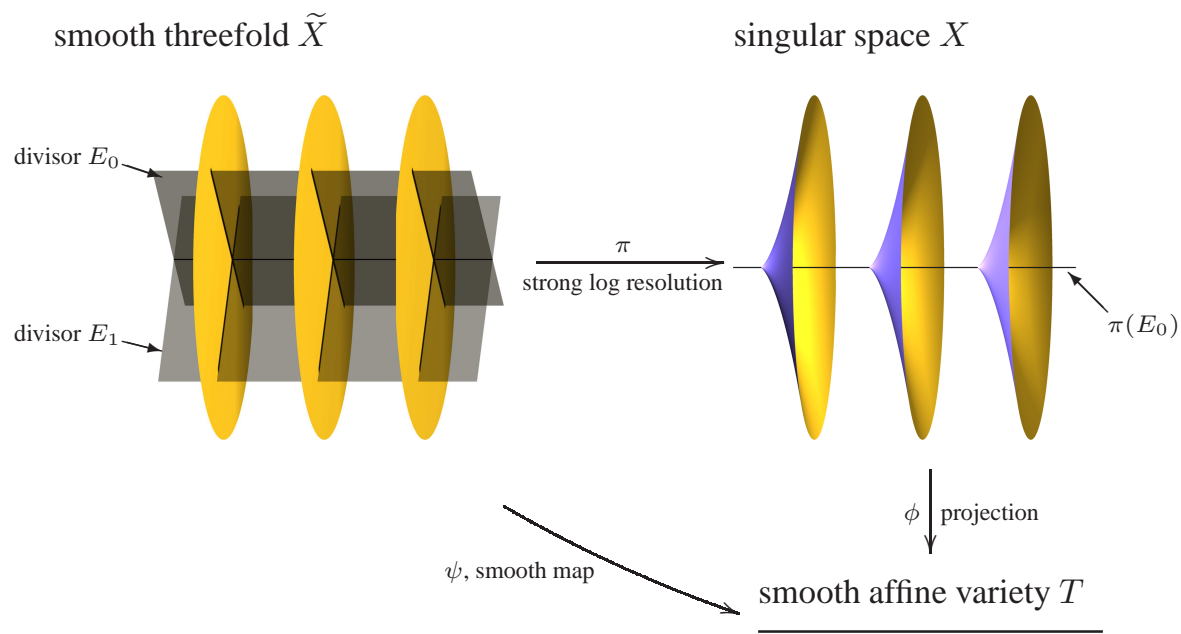

The figure sketches a situation where Assumption 17.13 holds, in the simple case where $\lfloor D\rfloor=0$ and $\widetilde{\Delta}^{\prime}=E_{0} \cup E_{1}$. The morphism $\pi_{t}$ maps the curves $E_{0, t}$ to isolated singularities of $\phi$-fibres. The morphism $\psi$ is an snc morphism of the pair $(\widetilde{X}, \widetilde{D})$.

FIGURE 4. Situation after projection to $\pi\left(E_{0}\right)$

Notation 17.14. If $t \in T$ is any point, we consider the varieties $X_{t}:=\phi^{-1}(t), \widetilde{X}_{t}:=$ $\psi^{-1}(t)$, divisors $E_{t}:=E \cap \widetilde{X}_{t}, E_{0, t}:=E_{0} \cap \widetilde{X}_{t}, \widetilde{D}_{t}^{\prime}:=\widetilde{D}^{\prime} \cap \widetilde{X}_{t}, \ldots$, and morphisms $\pi_{t}:=\left.\pi\right|_{\widetilde{X}_{t}}: \widetilde{X}_{t} \rightarrow X_{t}, \ldots$

The present setup is sketched in Figure 4 . We will now show that all assumptions made in Theorem 16.2 also hold for the general fibre $\widetilde{X}_{t}$ of $\psi$. Better still, the morphism $\pi_{t}$ maps $E_{0, t}$ to a point. In Section 17.C.5 we will then be able to apply Corollary 14.2 to fibres of $\psi$. A vanishing result for cohomology with support will follow.

Claim 17.15. If $t \in T$ is a general point, then $\left(X_{t}, D_{t}\right)$ is a $\log$ canonical pair, and the morphism $\pi_{t}: \widetilde{X}_{t} \rightarrow X_{t}$ is a $\log$ resolution of the pair $\left(X_{t}, D_{t}\right)$ which has $E_{t}$ as its exceptional set and contracts the divisor $E_{0, t}$ to a point. Further, we have

$$
\widetilde{D}_{t}^{\prime}=\left(\operatorname{supp} E_{t}\right) \cup \pi_{t}^{-1}\left(\operatorname{supp}\left\lfloor D_{t}\right\rfloor\right) .
$$

Proof. The fact that $\pi_{t}\left(E_{0, t}\right)$ is a point is immediate from Assumption17.12 The remaining assertions follow from Lemma 2.22 and 2.23 on page 9

Again, shrinking $T$ and $X$ to simplify notation, we may assume without loss of generality that the following holds.

Additional Assumption 17.16. The conclusion of Claim 17.15 holds for all points $t \in T$.

17.C.5. Vanishing results for relative differentials. Claim 17.15 asserts that $\pi$ maps $E_{0, t}:=E_{0} \cap \widetilde{X}_{t}$ to a single point. The Steenbrink-type vanishing result for cohomology with supports, Corollary 14.2, therefore guarantees the vanishing of cohomology groups with support on $E_{0, t}$, for sheaves of differentials on $\widetilde{X}_{t}$.

Claim 17.17. If $t \in T$ is any point, and if $z \in \pi\left(E_{0}\right)$ is the unique point with $\phi(z)=t$, then $H_{\pi^{-1}(z)}^{1}\left(\widetilde{X}_{t}, \Omega_{\widetilde{X}_{t}}^{q}\left(\log \widetilde{D}_{t}^{\prime}\right)\right)=0$ for all numbers $0 \leq q \leq \operatorname{dim} X-\operatorname{dim} T$. 
Claim 17.17 and the Generic Base Change Theorem for cohomology with supports, Theorem 15.1, then immediately give the following vanishing of cohomology with support on $E_{0}$, for sheaves of relative differentials on $\widetilde{X}$, possibly after shrinking $T$.

Claim 17.18. We have $H_{\pi^{-1}\left(\pi\left(E_{0}\right)\right)}^{1}\left(\widetilde{X}, \Omega_{\widetilde{X} / T}^{q}\left(\log \widetilde{D}^{\prime}\right)\right)=0$ for all numbers $1 \leq q \leq$ $\operatorname{dim} X-\operatorname{dim} T$.

17.C.6. Relative differential sequences, completion of the proof. By Assumption 17.13. the divisor $\widetilde{D}^{\prime}$ is relatively snc over $T$. As we have recalled in Section 10.A this implies the existence of a filtration

$$
\Omega_{\widetilde{X}^{p}}\left(\log \widetilde{D}^{\prime}\right)=\mathscr{F}^{0} \supseteq \mathscr{F}^{1} \supseteq \cdots \supseteq \mathscr{F}^{p} \supseteq \mathscr{F}^{p+1}=0
$$

with quotients

$$
0 \longrightarrow \mathscr{F}^{r+1} \longrightarrow \mathscr{F}^{r} \longrightarrow \psi^{*} \Omega_{T}^{r} \otimes \Omega_{\widetilde{X} / T}^{p-r}\left(\log \widetilde{D}^{\prime}\right) \longrightarrow 0 .
$$

By Assumption 17.12, the pull-backs $\psi^{*} \Omega_{T}^{r}$ are trivial vector bundles, and the sheaves $\mathscr{F}^{r} / \mathscr{F}^{r+1}$ are therefore isomorphic to direct sums of several copies of $\Omega_{\widetilde{X} / T}^{p-r}\left(\log \widetilde{D}^{\prime}\right)$. For simplicity, we will therefore use the somewhat sloppy notation

$$
\mathscr{F}^{r} / \mathscr{F}^{r+1}=\Omega_{\widetilde{X} / T}^{p-r}\left(\log \widetilde{D}^{\prime}\right)^{\oplus \bullet}
$$

Recall Observation 17.10, which asserts that to prove Theorem 16.2 it suffices to show that the injective restriction map

$$
t: H^{0}\left(\widetilde{X}, \mathscr{F}^{0}\right) \rightarrow H^{0}(\underbrace{\tilde{X} \backslash \pi^{-1}\left(\pi\left(E_{0}\right)\right)}_{=: \widetilde{X}^{\circ}}, \mathscr{F}^{0}) .
$$

is surjective. To this end, we consider the long exact cohomology sequences associated with 17.18.1, and with its restriction to $\tilde{X}^{\circ}=\tilde{X} \backslash \pi^{-1}\left(\pi\left(E_{0}\right)\right)$. Table 2 on the facing page shows an excerpt of the commutative diagram that is relevant to our discussion.

Note that the restriction map $t$ of 17.18.2 appears under the name $b_{0}$ in Table 2 While it is clear that the restriction morphisms $a_{r}, b_{r}$ and $c_{r}$ are injective, surjectivity of $c_{r}$ and injectivity of $f_{r}$ both follow from Claim 17.18 when one applies the standard long exact sequence for cohomology with supports, [Har77. III Ex. 2.3(e)], to the sheaf $\mathscr{A}:=\Omega_{\widetilde{X} / T}^{p-r}\left(\log \widetilde{D}^{\prime}\right)^{\oplus \bullet}$,

$$
\begin{aligned}
& \underbrace{H_{\pi^{-1}\left(\pi\left(E_{0}\right)\right)}^{0}(\tilde{X}, \mathscr{A})}_{=\{0\} \text { because } \mathscr{A} \text { is torsion free }} \rightarrow H^{0}(\tilde{X}, \mathscr{A}) \stackrel{c_{r}}{\rightarrow} H^{0}\left(\widetilde{\left.X^{\circ}, \mathscr{A}\right)}\right. \\
& \rightarrow \underbrace{H_{\pi^{-1}\left(\pi\left(E_{0}\right)\right)}^{1}(\widetilde{X}, \mathscr{A})}_{=\{0\} \text { by Claim } 17.18} \rightarrow H^{1}(\widetilde{X}, \mathscr{A}) \stackrel{f_{r}}{\rightarrow} H^{1}\left(\widetilde{X}^{\circ}, \mathscr{A}\right) \rightarrow \cdots
\end{aligned}
$$

In this setting, surjectivity of the restriction map $t=b_{0}$ follows from an inductive argument. More precisely, we use descending induction to show that the following stronger statement holds true.

Claim 17.19. For all numbers $r \leq p$ the following two statements hold true.

(17.19.1) The map $b_{r}: H^{0}\left(\widetilde{X}, \mathscr{F}^{r}\right) \rightarrow H^{0}\left(\widetilde{X}^{\circ}, \mathscr{F}^{r}\right)$ is surjective.

(17.19.2) The map $e_{r}: H^{1}\left(\tilde{X}, \mathscr{F}^{r}\right) \rightarrow H^{1}\left(\widetilde{X}^{\circ}, \mathscr{F}^{r}\right)$ is injective. 


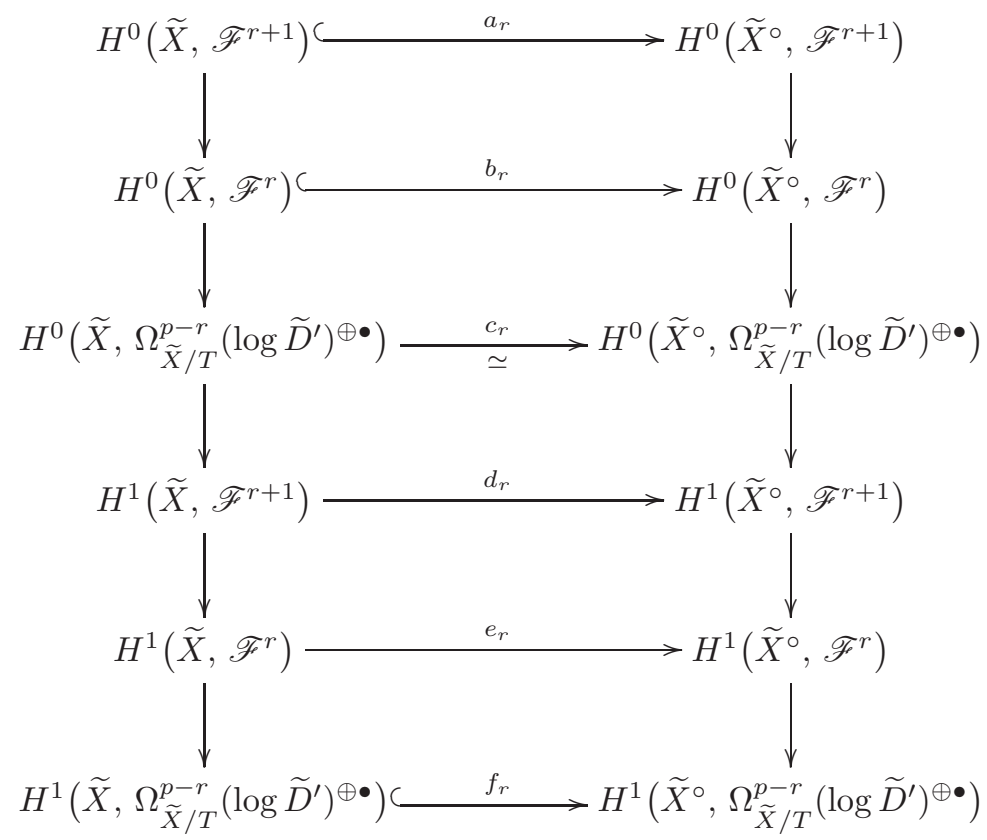

TABLE 2. Long exact cohomology sequences for relative differentials

Proof of Claim 17.19 start of induction: $r=p$. In this setup, $\mathscr{F}^{r+1}=0$, the map $d_{r}$ is obviously injective, and $a_{r}$ is surjective. Statement (17.19.1) follows when one applies the Four-Lemma for Surjectivity, Lemma C.2 to the first four rows of Table 2 Statement (17.19.2) then immediately follows when one applies the Four-Lemma for Injectivity, LemmaC.1 to the last four rows of Table 2 .

Proof of Claim 17.19 inductive step. Let $r<p$ be any given number and assume that Statements (17.19.1) and (17.19.2) were known for all indices larger than $r$. Since $d_{r}=$ $e_{r+1}$ is injective by assumption, and $a_{r}=b_{r+1}$ is surjective, we argue as in case $r=p$ above: Statement (17.19.1) follows from the Four-Lemma for Surjectivity, Lemma C.2, and the first four rows of Table 2. Statement (17.19.2) follows from the Four-Lemma for Injectivity, Lemma C.1 and the last four rows of Table 2

Summary. In summary, we have shown surjectivity of the restriction map $t=b_{0}$. This completes the proof of Theorem 16.2 and hence of Theorem 16.1

\section{PART VI. PROOF OF THE EXTENSION THEOREM 1.5}

\section{Proof of TheOREM 1.5, IDEA OF ProOF}

To explain the main ideas in the proof of the Extension Theorem 1.5] consider the case where $X$ is a klt space that contains a single isolated singularity, and let $\pi: \widetilde{X} \rightarrow X$ be a strong $\log$ resolution of the pair $(X, \emptyset)$, with $\pi$-exceptional divisor $E \subset \widetilde{X}$. As explained in Remark 1.5 .2 on page 4 to prove Theorem 1.5 we need to show that for any open set $U \subseteq X$ with preimage $\tilde{U}$, any differential form defined on $\widetilde{U} \backslash E$ extends across $E$, to give a differential form defined on all of $\widetilde{U}$. To this end, fix an open set $U \subseteq X$ and let 
snc surface pair $\left(X_{0}, E_{1}+E_{2}\right) \quad$ dlt surface pair $\left(X_{1}, E_{2,1}\right)$
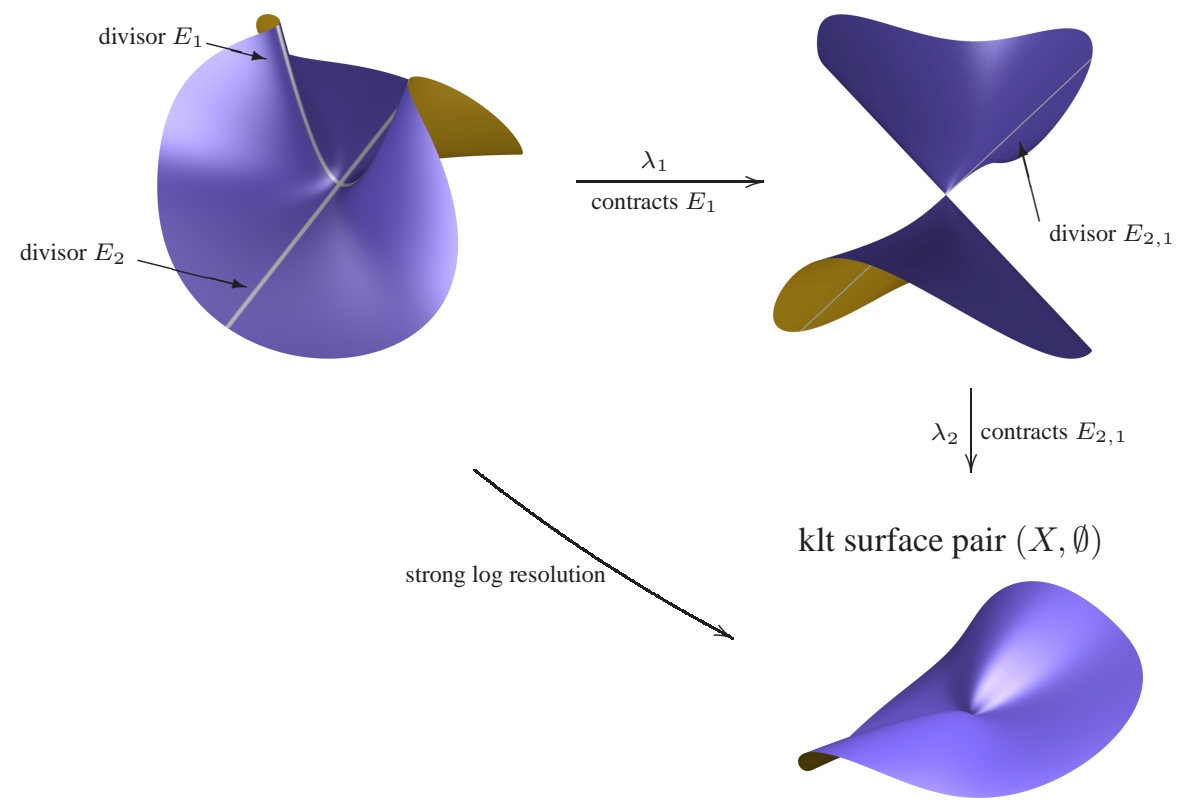

This sketch shows the strong log resolution of an isolated klt surface singularity, and the decomposition of the strong log resolution given by the minimal model program of the snc pair $\left(X_{0}, E_{1}+E_{2}\right)$. The example is taken from [Bau07.

FIGURE 5. Strong log resolution of an isolated klt surface singularity

$\sigma \in H^{0}\left(\widetilde{U} \backslash E, \Omega_{\widetilde{X}}^{p}\right)$ be any form. For simplicity of notation, we assume without loss of generality that $U=X$. Also, we consider only the case where $p>1$ in this sketch.

As a first step towards the extension of $\sigma$, we have seen in Theorem 16.1 on page 44 that $\sigma$ extends as a form with logarithmic poles along $E$, say $\bar{\sigma} \in H^{0}\left(\tilde{X} \backslash E, \Omega_{\widetilde{X}}^{p}(\log E)\right)$. Next, we need to show that $\bar{\sigma}$ really does not have any poles along $E$. To motivate the strategy of proof, we consider two simple cases first.

18.A. The case where $E$ is irreducible. Assume that $E$ is irreducible. To show that $\bar{\sigma}$ does not have any logarithmic poles along $E$, recall from Fact 11.3 on page 30 that it suffices to show that $\bar{\sigma}$ is in the kernel of the residue map

$$
\rho^{p}: H^{0}\left(\tilde{X}, \Omega_{\widetilde{X}}^{p}(\log E)\right) \rightarrow H^{0}\left(E, \Omega_{E}^{p-1}\right) .
$$

On the other hand, we know from a result of Hacon-McKernan, [HM07, Cor. 1.5(2)], that $E$ is rationally connected, so that $h^{0}\left(E, \Omega_{E}^{p-1}\right)=0$. This clearly shows that $\bar{\sigma}$ is in the kernel of $\rho^{p}$ and completes the proof when $E$ is irreducible.

18.B. The case where $(\widetilde{X}, E)$ has a simple mmp. In general, the divisor $E$ need not be irreducible. Let us therefore consider the next difficult case that where $E$ is reducible with two components, say $E=E_{1} \cup E_{2}$. The strong $\log$ resolution $\pi$ will then factor via a $\pi$-relative minimal model program of the pair $(\widetilde{X}, E)$, which we assume for simplicity to 
have the following particularly special form, sketched also in Figure 5 on the facing page

$$
\tilde{X}=X_{0} \underset{\text { contracts } E_{1} \text { to a point }}{\longrightarrow} X_{1} \underset{\text { contracts } E_{2,1}:=\left(\lambda_{1}\right)_{*}\left(E_{2}\right) \text { to a point }}{\longrightarrow} X \text {. }
$$

In this setting, the arguments of Section $18 . \mathrm{A}$ apply to show that $\bar{\sigma}$ has no poles along the divisor $E_{1}$. To show that $\bar{\sigma}$ does not have any poles along the remaining component $E_{2}$, observe that it suffices to consider the induced reflexive form on the possibly singular space $X_{1}$, say $\bar{\sigma}_{1} \in H^{0}\left(X_{1}, \Omega_{X_{1}}^{[p]}\left(\log E_{2,1}\right)\right)$, where $E_{2,1}:=\left(\lambda_{1}\right)_{*}\left(E_{2}\right)$, and to show that $\bar{\sigma}_{1}$ does not have any poles along $E_{2,1}$. For that, we follow the arguments of Section 18.A once more, carefully accounting for the singularities of the pair $\left(X_{1}, E_{2,1}\right)$.

The pair $\left(X_{1}, E_{2,1}\right)$ is dlt, and it follows that the divisor $E_{2,1}$ is necessarily normal, [KM98, Cor. 5.52]. Using the residue map for reflexive differentials on dlt pairs that was constructed in Theorem 11.7 on page 31 .

$$
\rho^{[p]}: H^{0}\left(X_{1}, \Omega_{X_{1}}^{[p]}\left(\log E_{2,1}\right)\right) \rightarrow H^{0}\left(E_{2,1}, \Omega_{E_{2,1}}^{[p-1]}\right),
$$

we have seen in Remark 11.8 that it suffices to show that $\rho^{[p]}\left(\bar{\sigma}_{1}\right)=0$. Because the morphism $\lambda_{2}$ contracts the divisor $E_{2,1}$ to a point, the result of Hacon-McKernan will again apply to show that $E_{2,1}$ is rationally connected. Even though there are numerous examples of rationally connected spaces that carry non-trivial reflexive forms, we claim that in our special setup we do have the vanishing

$$
h^{0}\left(E_{2,1}, \Omega_{E_{2,1}}^{[p-1]}\right)=0 .
$$

Recall from the adjunction theory for Weil divisors on normal spaces, [Kol92, Chapt. 16 and Prop. 16.5] and [Cor07, Sect. 3.9 and Glossary], that there exists a Weil divisor $D_{E}$ on the normal variety $E_{2,1}$ which makes the pair $\left(E_{2,1}, D_{E}\right)$ klt. Now, if we knew that the extension theorem would hold for the pair $\left(E_{2,1}, D_{E}\right)$, we can prove the vanishing (18.1), arguing exactly as in the proof of Theorem 5.1 on page 15 , where we show the non-existence of reflexive forms on rationally connected klt spaces as a corollary of the Extension Theorem 1.5. Since $\operatorname{dim} E_{2,1}<\operatorname{dim} X$, this suggests an inductive proof, beginning with easy-to-prove extension theorems for reflexive forms on surfaces, and working our way up to higher-dimensional varieties. The proof of Theorem 1.5 follows this inductive pattern.

18.C. The general case. The assumptions made in Sections 18.A 18.B of course do not hold in general. To handle the general case, we need to work with pairs $(X, D)$ where $D$ is not necessarily empty, the $\pi$-relative minimal model program might involve flips, and the singularities of $X$ need not be isolated. All this leads to a slightly protracted inductive argument, which is outlined in all detail in the next section.

\section{Proof of THEOREM 1.5, OVERVIEW OF THE PROOF}

19.A. Notation used in the induction. We aim to prove Theorem 1.5 for $\log$ canonical pairs of arbitrary dimension. As we will argue by induction, we often need to prove statements of the form "If Proposition 19.3 holds for all pairs of a given dimension $n \geq 2$, then Proposition 19.1 will hold for all pairs of the same dimension $n$ ". It makes sense to introduce the following shorthand notation for this,

$$
\forall n \geq 2: \text { Proposition } 19.3(n) \Longrightarrow \operatorname{Proposition~} 19.1(n) .
$$


Likewise, to say that "Given any number $n \geq 2$, if Proposition 19.4 holds for all pairs of dimension $n^{\prime} \leq n$, then Proposition 19.1 will hold for all pairs of dimension $n+1$ ", we will write

$$
\left.\forall n \geq 2:\left(\text { Proposition } 19.4\left(n^{\prime}\right), \forall n^{\prime} \leq n\right) \Longrightarrow \text { Proposition 19.3 } n+1\right)
$$

If we want to say that Proposition 19.3 holds for surface pairs, we will often write

$$
\text { Proposition } 19.3(n=2) \text {. }
$$

19.B. Theorems and propositions that appear in the induction. Before giving an overview of the induction process and listing the implications that we will prove, we have gathered in this section a complete list of the theorems and propositions that will play a role in the proof.

In the setup of the Extension Theorem 1.5 we have seen in Theorem 16.1 on page 44 that any differential form on $\widetilde{X}$ which is defined away from the $\pi$-exceptional set $E$ extends as a form with logarithmic poles along $E$. As a consequence, we will see in Section 20 that to prove the Extension Theorem 1.5, it suffices to show that the following Proposition holds for all numbers $n \geq 2$.

Proposition 19.1 (Non-existence of logarithmic poles for pairs of dimension $n$ ). Let $(X, D)$ be a log canonical pair of dimension $\operatorname{dim} X=n$, and let $\pi: \widetilde{X} \rightarrow X$ be a $\log$ resolution of $(X, D)$, with exceptional set $E \subset \widetilde{X}$. Consider the two divisors

$$
\begin{aligned}
\widetilde{D} & :=\text { largest reduced divisor contained in } \operatorname{supp} \pi^{-1} \text { (non-klt locus), } \\
\widetilde{D}^{\prime} & :=\operatorname{supp}\left(E+\pi^{-1} \operatorname{supp}\lfloor D\rfloor\right)
\end{aligned}
$$

and observe that $\widetilde{D} \subseteq \widetilde{D}^{\prime}$. Then the natural injection

$$
H^{0}\left(\widetilde{X}, \Omega_{\widetilde{X}}^{p}(\log \widetilde{D})\right) \rightarrow H^{0}\left(\widetilde{X}, \Omega_{\widetilde{X}}^{p}\left(\log \widetilde{D}^{\prime}\right)\right)
$$

is in fact isomorphic.

Remark 19.1.2. Recall from Lemma 2.15 on page 7 that the pair $\left(\widetilde{X}, \widetilde{D}^{\prime}\right)$ is reduced and snc. Being a subdivisor of a divisor with simple normal crossing support, the pair $(\widetilde{X}, \widetilde{D})$ is likewise reduced and snc. In particular, it follows that the sheaves $\Omega_{\widetilde{X}}^{p}(\log \widetilde{D})$ and $\Omega_{\widetilde{X}}^{p}\left(\log \widetilde{D}^{\prime}\right)$ mentioned in (19.1.1) are locally free.

As indicated in Section 18, we aim to prove Proposition 19.1 by using the $\pi$-relative minimal model program of the pair $(X, E)$, in order to contract one irreducible component of $E$ at a time. The proof of Proposition 19.1 will then depend on the following statements, which assert that differential forms extend across irreducible, contractible divisors. For technical reasons, we handle the cases of 1 -forms and of $p$-forms separately.

Proposition 19.2 (Extension of 1-forms over contractible divisors). Let $(X, D)$ be a dlt pair of dimension $\operatorname{dim} X \geq 2$, where $X$ is $\mathbb{Q}$-factorial, and let $\lambda: X \rightarrow X_{\lambda}$ be a divisorial contraction of a minimal model program associated with the pair $(X, D)$, contracting an irreducible divisor $D_{0} \subseteq \operatorname{supp}\lfloor D\rfloor$. Then the natural injection

$$
H^{0}\left(X, \Omega_{X}^{[1]}\right) \rightarrow H^{0}\left(X, \Omega_{X}^{[1]}\left(\log D_{0}\right)\right)
$$

is isomorphic. 
Proposition 19.3 (Extension of $p$-forms over contractible divisors in dimension $n$ ). Let $(X, D)$ be a dlt pair of dimension $\operatorname{dim} X=n$, where $X$ is $\mathbb{Q}$-factorial, and let $\lambda$ : $X \rightarrow X_{\lambda}$ be a divisorial contraction of a minimal model program associated with the pair $(X, D)$, contracting an irreducible divisor $D_{0} \subseteq \operatorname{supp}\lfloor D\rfloor$. Then the natural injection

$$
H^{0}\left(X, \Omega_{X}^{[p]}\right) \rightarrow H^{0}\left(X, \Omega_{X}^{[p]}\left(\log D_{0}\right)\right)
$$

is isomorphic for all numbers $1<p \leq \operatorname{dim} X$.

Finally, we have seen in Section 18 that the non-existence of reflexive differentials on rationally chain connected klt spaces enters the proof of Proposition 19.2. The relevant statement is this, compare also Theorem 5.1 on page 15

Proposition 19.4 (Reflexive differentials on rcc pairs of dimension $n$ ). Let $(X, D)$ be a klt pair of dimension $\operatorname{dim} X=n$. If $X$ is rationally chain connected, then $X$ is rationally connected and $H^{0}\left(X, \Omega_{X}^{[p]}\right)=0$ for all numbers $1 \leq p \leq \operatorname{dim} X$.

19.C. Overview of the induction process. Using the notation introduced in Section 19. A on page 53 . Table 3 shows the structure of the inductive proof of the Extension Theorem 1.5. The steps are carried out in Sections 20 25, respectively, Step 5 being by far the most involved.

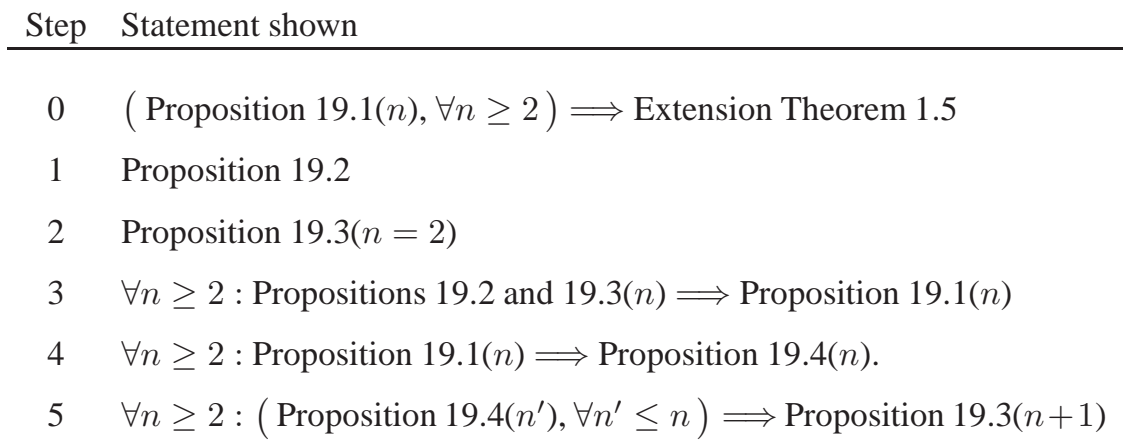

TABLE 3. Overview of the induction used to prove Theorem 1.5.

\section{Step 0 IN THE PROOF OF TheOREM 1.5}

Assuming that Proposition 19.1 holds for log canonical pairs of arbitrary dimension, we show in this section that the Extension Theorem 1.5 follows. To prove Theorem 1.5 let $(X, D)$ be an arbitrary lc pair, and let $\pi: \widetilde{X} \rightarrow X$ be an arbitrary log resolution, with exceptional set $E \subset \widetilde{X}$. Following Remark 1.5.2, we need to show that for any open set $U \subseteq X$ with preimage $\widetilde{U} \subseteq \widetilde{X}$, any differential form

$$
\sigma \in H^{0}\left(\widetilde{U} \backslash E, \Omega_{\widetilde{X}}^{p}(\log \widetilde{D})\right) \quad \text { extends to a form } \quad \widetilde{\sigma} \in H^{0}\left(\widetilde{U}, \Omega_{\widetilde{X}}^{p}(\log \widetilde{D})\right),
$$

where $\widetilde{D}$ is the divisor on $\widetilde{X}$ defined in Theorem 1.5

As a first step in this direction, given an open set $U$ and a form $\sigma$, apply Theorem 16.1 to the pair $(U, D)$, to obtain an extension of $\sigma$ to a differential form

$$
\widetilde{\sigma}^{\prime} \in H^{0}\left(\widetilde{U}, \Omega_{\widetilde{X}}^{p}\left(\log \widetilde{D}^{\prime}\right)\right),
$$


where $\widetilde{D}^{\prime} \supseteq \widetilde{D}$ is the larger divisor defined in Theorem 16.1 An application of Proposition 19.1 to the pair $(U, D)$ will then show that $\widetilde{\sigma}^{\prime}$ in fact does not have any logarithmic poles along the difference divisor $\widetilde{D}^{\prime}-\widetilde{D}$. This finishes Step 0 in the proof of Theorem 1.5

\section{SteP 1 IN THE PROOF OF THEOREM 1.5}

In this section, we will prove Proposition 19.2. We maintain the assumptions and the notation of the proposition. As we will see, the assertion follows from the Extension Theorem [GKK10, Thm. 1.1] for 1-forms on reduced, log canonical pairs. Let $r: \widetilde{X} \rightarrow X_{\lambda}$ be a $\log$ resolution of $\left(X_{\lambda}, \emptyset\right)$ that factors through $X$. We obtain a diagram

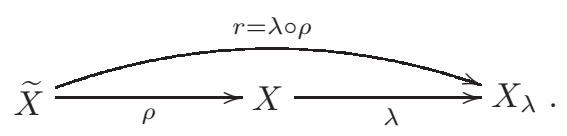

Now let $\sigma \in H^{0}\left(X, \Omega_{X}^{[1]}\left(\log D_{0}\right)\right)$ be any given reflexive form on $X$, possibly with logarithmic poles along $D_{0}$. Since the divisor $D_{0}$ is contracted by $\lambda$, the form $\sigma$ induces a reflexive form $\sigma_{\lambda} \in H^{0}\left(X_{\lambda}, \Omega_{X_{\lambda}}^{[1]}\right)$, without any poles.

Claim 21.1. The direct image sheaf $r_{*} \Omega_{\widetilde{X}}^{1}$ is reflexive. In particular, the pull-back of $\sigma_{\lambda}$ to $\widetilde{X}$ by $r$ defines a regular form $\widetilde{\sigma} \in H^{0}\left(\widetilde{X}, \Omega_{\widetilde{X}}^{1}\right)$, which agrees with the pull-back of $\sigma$ by $\rho$ wherever the morphism $\rho$ is isomorphic.

Remark 21.1.1. We refer to Remark 1.5 .2 on page 4 for an explanation why reflexivity of $r_{*}\left(\Omega_{\widetilde{X}}^{p}\right)$ and the extension of pull-back forms are equivalent.

Proof of Claim 21.1] Let $r^{\prime}: \widetilde{X}^{\prime} \rightarrow X_{\lambda}$ be any strong log resolution of the pair $\left(X_{\lambda}, \emptyset\right)$. The Comparison Lemma, [GKK10, Lem. 2.13], then asserts that the direct image sheaf $r_{*}\left(\Omega_{\widetilde{X}}^{1}\right)$ is reflexive if $\left(r^{\prime}\right)_{*}\left(\Omega_{\widetilde{X}}^{1}\right)$ is reflexive. Reflexivity of $\left(r^{\prime}\right)_{*}\left(\Omega_{\tilde{X}}^{1}\right)$, however, follows from the Extension Theorem [GKK10, Thm. 1.1] for 1-forms on reduced, log canonical pairs once we show that $\left(X_{\lambda}, \emptyset\right)$ is klt.

To this end, recall from [KM98, Sect. 3.31] that $X_{\lambda}$ is $\mathbb{Q}$-factorial, and that the pair $\left(X_{\lambda}, \lambda_{*} D\right)$ is again dlt. The fact that $\left(X_{\lambda}, \emptyset\right)$ is klt then follows from [KM98, Cor. 2.39 and Prop. 2.41] because $\lambda_{*} D$ will be $\mathbb{Q}$-Cartier. This completes the proof of Claim 21.1.

By Claim 21.1, the pull-back form $\widetilde{\sigma}$ does not have any poles along the strict transform of $D_{0}$, this shows that $\sigma$ does not have any poles along $D_{0}$, as claimed. This completes the proof of Proposition 19.2 and therefore finishes Step 1 in the proof of Theorem 1.5 .

\section{STEP 2 IN THE PROOF OF THEOREM 1.5}

We will now prove Proposition $19.3 n=2$ ). Thus $n=\operatorname{dim} X=2$ and $p=2$. Let $\sigma \in H^{0}\left(X, \Omega_{X}^{[2]}\left(\log D_{0}\right)\right)$ be any given reflexive form on $X$. Recall from Theorem 11.7 on page 31 that there exists a residue map for reflexive differentials,

$$
\rho^{[2]}: \Omega_{X}^{[2]}\left(\log D_{0}\right) \rightarrow \Omega_{D_{0}}^{[1]},
$$

which agrees with the residue map of the standard residue sequence 11.1) wherever the dlt pair $\left(X, D_{0}\right)$ is snc. Also, recall from [KM98, Cor. 2.39(1) and Cor. 5.52] that $\left(X, D_{0}\right)$ is dlt, and that $D_{0}$ is normal. The divisor $D_{0}$ is therefore a smooth curve, and $\Omega_{D_{0}}^{[1]}=\Omega_{D_{0}}^{1}$. Adjunction together with the fact that $-\left(K_{X}+D\right)$ is $\lambda$-ample implies that $D_{0} \simeq \mathbb{P}^{1}$. The space of differentials of $D_{0}$ is therefore trivial, $H^{0}\left(D_{0}, \Omega_{D_{0}}^{1}\right)=0$. In particular, it follows 
that $\rho^{[2]}(\sigma)=0$. It follows from the fact that the residue map acts as a test for logarithmic poles, see Remark 11.8, that $\sigma \in H^{0}\left(X, \Omega_{X}^{[2]}\right)$, as claimed. This completes the proof of Proposition 19.3 $n=2)$ and therefore finishes Step 2 in the proof of Theorem 1.5 .

\section{STEP 3 IN THE PROOF OF THEOREM 1.5}

Let $(X, D)$ be a $\log$ canonical pair of dimension $n$, and let $\pi: \tilde{X} \rightarrow X$ be a log resolution. We need to show surjectivity of the natural inclusion map 19.1.1), assuming that Proposition $19.3 n$ ) holds. Observing that the statement of Proposition $19.1(n)$ is local on $X$, we may assume that the following holds.

Additional Assumption 23.1. The space $X$ is affine.

Furthermore, if $\widetilde{D}_{0}^{\prime} \subset \widetilde{D}^{\prime}$ is any irreducible component such that $\pi\left(\widetilde{D}_{0}^{\prime}\right)$ is contained in the non-klt locus of $(X, D)$, then $\widetilde{D}_{0}^{\prime}$ is also contained in $\widetilde{D}$. We may therefore assume without loss of generality that the following holds

Additional Assumption 23.2. The pair $(X, D)$ is klt.

Let $E \subset \widetilde{X}$ denote the $\pi$-exceptional set. In order to prove surjectivity of 19.1.1) it is equivalent to show that the natural map

$$
H^{0}\left(\widetilde{X}, \Omega_{\widetilde{X}}^{p}\right) \rightarrow H^{0}\left(\widetilde{X}, \Omega_{\widetilde{X}}^{p}(\log E)\right)
$$

is surjective. By the definition of klt there exist effective $\pi$-exceptional divisors $F$ and $G$ without common components such that $\lfloor F\rfloor=0$ and such that the following $\mathbb{Q}$-linear equivalence holds:

$$
K_{\widetilde{X}}+\pi_{*}^{-1} D+F \sim_{\mathbb{Q}} \pi^{*}\left(K_{X}+D\right)+G .
$$

Let $\Delta_{\varepsilon}=\pi_{*}^{-1} D+F+\varepsilon E$. Choosing a small enough $0<\varepsilon \ll 1$ we may assume that the pair $\left(X, \Delta_{\varepsilon}\right)$ is klt. Let $H \subset \widetilde{X}$ be a $\pi$-ample divisor such that $\left(X, \Delta_{\varepsilon}+H\right)$ is still klt and $K_{\widetilde{X}}+\Delta_{\varepsilon}+H$ is $\pi$-nef. We may then run the $\pi$-relative $\left(\widetilde{X}, \Delta_{\epsilon}\right)$ minimal model program with scaling of $H$ cf. [BCHM10, Cor. 1.4.2], [HK10, Thms. 5.54,5.63]. Therefore there exists a commutative diagram

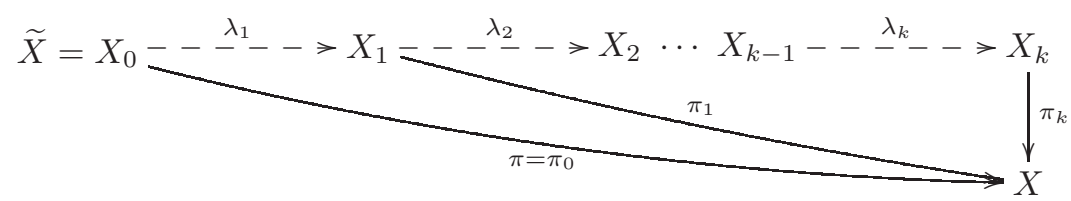

where the $\lambda_{i}$ are either divisorial contractions or flips. The spaces $X_{i}$ are normal, $\mathbb{Q}$ factorial, and if $\Delta_{i} \subset X_{i}$ denotes the cycle-theoretic image of $\Delta_{\varepsilon}$, then the pairs $\left(X_{i}, \Delta_{i}\right)$ are klt for all $i$. The minimal model program terminates with a pair $\left(X_{k}, \Delta_{k}\right)$ whose associated $\mathbb{Q}$-divisor $K_{X_{k}}+\Delta_{k}$ is $\pi_{k}$-nef.

Notation 23.3. Given any $0 \leq i \leq k$, let $E_{i}$ (respectively $G_{i}$ ) denote the cycle-theoretic image of $E$ (respectively $G$ ) on $X_{i}$.

Claim 23.4. The morphism $\pi_{k}$ is a small map. In particular, $E_{k}=\emptyset$.

Proof. It is clear from the construction that $\operatorname{supp} E_{i}$ is precisely the divisorial part of the $\pi_{i}$-exceptional set. Then the $\mathbb{Q}$-linear equivalence 23.2.1 implies that

$$
K_{X_{i}}+\Delta_{i} \sim_{\mathbb{Q}} \pi_{i}^{*}\left(K_{X}+D\right)+G_{i}+\varepsilon E_{i},
$$


where $G_{i}+\varepsilon E_{i}$ is effective and $\operatorname{supp}\left(G_{i}+\varepsilon E_{i}\right)=\operatorname{supp}\left(E_{i}\right)$. By item A.13 of Lemma A.1 this implies that $K_{X_{i}}+\Delta_{i}$ is not $\pi_{i}$-nef as long as $E_{i} \neq \emptyset$. It follows that $E_{k}$, the divisorial part of the $\pi_{k}$-exceptional set, is empty. This shows Claim 23.4

Let $\sigma \in H^{0}\left(\widetilde{X}, \Omega_{\widetilde{X}}^{p}(\log E)\right)$ be arbitrary. In order to complete Step 3 we need to show that $\sigma \in H^{0}\left(\tilde{X}, \Omega_{\tilde{X}}^{p}\right)$. Clearly, $\sigma$ induces reflexive forms $\sigma_{i} \in H^{0}\left(X_{i}, \Omega_{X_{i}}^{[p]}\left(\log E_{i}\right)\right)$, for all $i$. Since $E_{k}=\emptyset$, the reflexive form $\sigma_{k}$ does not have any logarithmic poles at all, that is, $\sigma_{k} \in H^{0}\left(X_{k}, \Omega_{X_{k}}^{[p]}\right)$. Now consider the map $\lambda_{k}: X_{k-1} \rightarrow X_{k}$.

- If $\lambda_{k}$ is a flip, then $\lambda_{k}$ is isomorphic in codimension one and it is clear that $\sigma_{k-1}$ again does not have logarithmic poles along any divisor.

- If $\lambda_{k}$ is a divisorial contraction, then the $\lambda_{k}$-exceptional set is contained in $E_{k-1}$, and either Proposition 19.2 or Proposition 19.3 $n$ ) applies to the map $\lambda_{k}$.

In either case, we obtain that $\sigma_{k-1} \in H^{0}\left(X_{k-1}, \Omega_{X_{k-1}}^{[p]}\right)$. Applying the same argument successively to $\lambda_{k}, \lambda_{k-1}, \ldots, \lambda_{1}$, we find that

$$
\sigma=\sigma_{0} \in H^{0}\left(\widetilde{X}, \Omega_{\widetilde{X}}^{p}\right)
$$

as claimed. This completes the proof of Proposition $19.1 \mathrm{n}$ ), once Propositions 19.2 and 19.3 $n$ ) are known to hold. Step 3 in the proof of Theorem 1.5 is thus finished.

\section{Step 4 IN THE PROOF OF THEOREM 1.5}

As in Proposition 19.4 let $(X, D)$ be a klt pair of dimension $\operatorname{dim} X=n$, and assume that $X$ is rationally chain connected. Assuming that Proposition $19.1(n)$ holds, we need to show that $X$ is rationally connected, and that $H^{0}\left(X, \Omega_{X}^{[p]}\right)=0$ for all numbers $1 \leq p \leq n$.

To this end, choose a strong $\log$ resolution $\pi: \widetilde{X} \rightarrow X$. Since klt pairs are also dlt, a result of Hacon and McKernan, [HM07, Cor. 1.5(2)], applies to show that $X$ and $\widetilde{X}$ are both rationally connected. In particular, recall from [Kol96, IV. Cor. 3.8] that

$$
H^{0}\left(\widetilde{X}, \Omega_{\widetilde{X}}^{p}\right)=0 \quad \forall p>0 .
$$

Next, let $\sigma \in H^{0}\left(X, \Omega_{X}^{[p]}\right)$ be any reflexive form. We need to show that $\sigma=0$. We consider the pull-back $\widetilde{\sigma}$, which is a differential form on $\widetilde{X}$, possibly with poles along the $\pi$-exceptional set $E$. However, since $(X, D)$ is klt, Theorem 16.1 from page 44 asserts that $\widetilde{\sigma}$ has at most logarithmic poles along $E$. Proposition $19.1(n)$ then applies to show that $\widetilde{\sigma}$ does in fact not have any poles at all. The assertion that $\widetilde{\sigma}=0$ then follows from 24.1 .

This shows that Proposition 19.4 $(n)$ follows from Proposition 19.1 $(n)$, and finishes Step 4 in the proof of Theorem 1.5

\section{Step 5 IN THE PROOF OF THEOREM 1.5}

25.A. Setup. Throughout the present Section 25, we consider the following setup.

Setup 25.1. Let $(X, D)$ be a dlt pair of dimension $\operatorname{dim} X=n+1>2$, where $X$ is is $\mathbb{Q}$-factorial, and let $\lambda: X \rightarrow X_{\lambda}$ be a divisorial contraction of a minimal model program associated with the pair $(X, D)$, contracting a divisor $D_{0} \subseteq \operatorname{supp}\lfloor D\rfloor$. We assume that Proposition $19.4 n^{\prime}$ ) holds for all numbers $n^{\prime} \leq n$.

Remark 25.2. Since $\lambda$ is a divisorial contraction of a minimal model program, the space $X_{\lambda}$ is again $\mathbb{Q}$-factorial, and the pair $\left(X_{\lambda}, \lambda_{*} D\right)$ is dlt. By $\mathbb{Q}$-factoriality, the pairs $\left(X, D_{0}\right)$, $(X, \emptyset)$ and $\left(X_{\lambda}, \emptyset\right)$ will likewise be dlt, [KM98, Cor. 2.39]. 
In order to prove Proposition $19.3(n+1)$ and thus to complete the proof of Theorem 1.5 we need to show that the natural inclusion map

$$
H^{0}\left(X, \Omega_{X}^{[p]}\right) \rightarrow H^{0}\left(X, \Omega_{X}^{[p]}\left(\log D_{0}\right)\right)
$$

is surjective for all numbers $1<p \leq \operatorname{dim} X$. To this end, let $\sigma \in H^{0}\left(X, \Omega_{X}^{[p]}\left(\log D_{0}\right)\right)$ be any given reflexive form on $X$. We show that the following holds.

Claim 25.3. The reflexive form $\sigma$ does not have any $\log$ poles, i.e., $\sigma \in H^{0}\left(X, \Omega_{X}^{[p]}\right)$.

We will prove Claim 25.3 in Sections $25 . \mathrm{E}$ and $25 . \mathrm{F}$ considering the cases where $\operatorname{dim} \lambda\left(D_{0}\right)=0$ and $\operatorname{dim} \lambda\left(D_{0}\right)>0$ separately. Before starting with the proof, we include preparatory Sections 25.B 25.D where we recall facts used in the proof, set up notation, and discuss the (non)existence of reflexive relative differentials on $D_{0}$.

25.B. Adjunction for the divisor $D_{0}$ in $\boldsymbol{X}$. By inversion of adjunction the support of the divisor $D_{0}$ is normal, [KM98, Cor. 5.52]. A technical difficulty occurring in our reasoning will be the fact that $D_{0}$ need not be Cartier, so that one cannot apply adjunction naïvely. It is generally not even true that $K_{D_{0}}$ or $K_{D_{0}}+\left.\left(D-D_{0}\right)\right|_{D_{0}}$ are $\mathbb{Q}$-Cartier. In particular, it does not make sense to say that $\left(D_{0},\left.\left(D-D_{0}\right)\right|_{D_{0}}\right)$ is klt. However, a more elaborate adjunction procedure, which involves a correction term Diff $D_{0}(0)$ that accounts for the failure of $D_{0}$ to be Cartier, is known to give the following.

Lemma 25.4 (Existence of a divisor making $D_{0} \mathrm{klt}$ ). There exists an effective $\mathbb{Q}$-Weil divisor Diff $D_{0}(0)$ on $D_{0}$ such that the pair $\left(D_{0}\right.$, Diff $\left.D_{0}(0)\right)$ is klt.

Proof. The divisor $D_{0}$ being normal, it follows from the Adjunction Formula for Weil divisors on normal spaces, [Ko192, Chapt. 16 and Prop. 16.5] see also [Cor07, Sect. 3.9 and Glossary], that there exists an effective $\mathbb{Q}$-Weil divisor Diff $D_{0}(0)$ on $D_{0}$ such that $K_{D_{0}}+$ Diff $_{D_{0}}(0)$ is $\mathbb{Q}$-Cartier and such that the following $\mathbb{Q}$-linear equivalence holds,

$$
K_{D_{0}}+\left.\operatorname{Diff}_{D_{0}}(0) \sim_{\mathbb{Q}}\left(K_{X}+D_{0}\right)\right|_{D_{0}} .
$$

Better still, since $D_{0}$ is irreducible, it follows from [KM98, Prop. 5.51] that the pair $\left(X, D_{0}\right)$ is actually plt, and [Kol92, Thm. 17.6] then gives that the pair $\left(D_{0}\right.$, Diff $\left.D_{0}(0)\right)$ is klt, as claimed.

25.C. Simplifications and notation. Observe that Claim 25.3 may be checked locally on $X_{\lambda}$. Better still, we may always replace $X_{\lambda}$ with an open subset $X_{\lambda}^{\circ} \subseteq X_{\lambda}$, as long as $X_{\lambda}^{\circ} \cap \pi\left(D_{0}\right) \neq \emptyset$. In complete analogy with the arguments of Section 17.C.2, we may therefore assume the following.

Additional Assumption 25.5. The variety $X_{\lambda}$ is affine. The image $T:=\lambda\left(D_{0}\right)$, taken with its reduced structure, is smooth and has a free sheaf of differentials, $\Omega_{T}^{1} \simeq \mathscr{O}_{T}^{\oplus \operatorname{dim} T}$.

Note that, as in Section 17.C.4 Assumption 25.5 allows to apply Noether normalisation to the affine variety $T$. Shrinking $X_{\lambda}$ further, and performing an étale base change, if necessary, Proposition 2.25 thus allows to assume the following.

Additional Assumption 25.6. There exists a commutative diagram of surjective morphisms

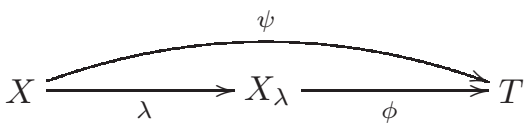

where the restriction $\left.\phi\right|_{\lambda\left(D_{0}\right)}: \lambda\left(D_{0}\right) \rightarrow T$ is isomorphic. 
Notation 25.7. If $t \in T$ is any point, we consider the scheme-theoretic fibres $X_{t}:=$ $\psi^{-1}(t), X_{\lambda, t}:=\phi^{-1}(t)$ and $D_{0, t}:=\left(\left.\psi\right|_{D_{0}}\right)^{-1}(t)$.

Shrinking $T$ - and thereby $X_{\lambda}$ - yet further, if necessary, the Cutting-Down Lemma 2.24 allows to assume that the appropriate fibre pairs are again dlt or klt. More precisely, we may assume that the following holds.

Additional Assumption 25.8. If $t \in T$ is any point, then $X_{t}$ and $X_{\lambda, t}$ are normal. The pairs $\left(X_{t}, D_{0, t}\right)$ and $\left(X_{\lambda, t}, \emptyset\right)$ are dlt, and $\left(D_{0, t}, \operatorname{Diff}_{D_{0}}(0) \cap D_{0, t}\right)$ are klt.

Remark 25.2 asserts that $\left(X, D_{0}\right)$ and $(X, \emptyset)$ are both dlt. Theorems 10.6 and 11.7 therefore apply, showing the existence of a filtration for relative reflexive differentials and the existence of a residue map over a suitable open set of $T$. Shrinking $T$ again, we may thus assume that the following holds.

Additional Assumption 25.9. The conclusions of Theorems 10.6 and 11.7 hold for the pairs $\left(X, D_{0}\right)$ and $(X, \emptyset)$ without further shrinking of $T$.

25.D. Vanishing of relative reflexive differentials on $D_{0}$. As we have seen in Section 18 , the non-existence of reflexive differentials on $D_{0}$ is an important ingredient in the proof of Theorem 1.5. Unlike the setup of Section 18, we do not assume that $D_{0}$ maps to a point, and a discussion of relative reflexive differentials is needed.

Lemma 25.10 (Vanishing of reflexive differentials on $D_{0, t}$ ). If $t \in T$ is any point, then $H^{0}\left(D_{0, t}, \Omega_{D_{0, t}}^{[q]}\right)=0$ for all $1 \leq q \leq n$.

Proof. Let $t \in T$ be any point and recall from [HM07, Cor. 1.3(2)] that $D_{0, t}$, which is a fibre of the map $\left.\lambda\right|_{X_{t}}: X_{t} \rightarrow X_{\lambda, t}$, is rationally chain connected. Since we argue under the inductive hypothesis that Proposition 19.4 $\left(n^{\prime}\right)$ holds for all numbers $n^{\prime} \leq n$ and since the pair $\left(D_{0, t}\right.$, Diff $\left._{D_{0}}(0) \cap D_{0, t}\right)$ is klt by Assumption 25.8, we obtain the vanishing $H^{0}\left(D_{0, t}, \Omega_{D_{0, t}}^{[q]}\right)=0$ for all $1 \leq q \leq n$, ending the proof.

Lemma 25.11 (Vanishing of relative reflexive differentials on $D_{0}$ ). We have $H^{0}\left(D_{0}, \Omega_{D_{0} / T}^{[q]}\right)=0$ for all $1 \leq q \leq n$.

Proof. We argue by contradiction and assume that there exists a non-zero section $\tau \in$ $H^{0}\left(D_{0}, \Omega_{D_{0} / T}^{[q]}\right)$. Let $D_{0}^{\circ} \subseteq D_{0}$ be the maximal open subset where the morphism $\left.\psi\right|_{D_{0}}$ is smooth, and let $Z:=D_{0} \backslash D_{0}^{\circ}$ be its complement. As before, set $D_{0, t}^{\circ}:=D_{0, t} \cap D_{0}^{\circ}$ and $Z_{t}:=D_{0, t} \cap Z$. Since $D_{0}$ is normal, it is clear that $\operatorname{codim}_{D_{0}} Z \geq 2$. If $t \in T$ is a general point, it is likewise clear that $\operatorname{codim}_{D_{0, t}} Z_{t} \geq 2$.

If $t \in T$ is general, the restriction of the non-zero section $\tau$ to $D_{0, t}^{\circ}$ does not vanish,

$$
\left.\tau\right|_{D_{0, t}^{\circ}} \in H^{0}\left(D_{0, t}^{\circ},\left.\Omega_{D_{0}^{\circ} / T}^{[q]}\right|_{D_{0, t}^{\circ}}\right) \backslash\{0\} .
$$

However, since $\left.\psi\right|_{D_{0}}$ is smooth along $D_{0, t}^{\circ}$, and since $\operatorname{codim}_{D_{0, t}} Z_{t} \geq 2$, we have isomorphisms

$$
H^{0}\left(D_{0, t}^{\circ},\left.\Omega_{D_{0}^{\circ} / T}^{[q]}\right|_{D_{0, t}^{\circ}}\right) \simeq H^{0}\left(D_{0, t}^{\circ}, \Omega_{D_{0, t}^{\circ}}^{q}\right) \simeq H^{0}\left(D_{0, t}, \Omega_{D_{0, t}}^{[q]}\right) .
$$

But Lemma 25.10 asserts that the right-hand side of 25.11.2 is zero, contradicting (25.11.1). The assumption that there exists a non-zero section $\tau$ is thus absurd, and Lemma 25.11 follows. 
25.E. Proof of Claim 25.3 if $\operatorname{dim} \pi\left(D_{0}\right)=0$. Theorem 11.7 assert that a residue map

$$
\rho^{[p]}: \Omega_{X}^{[p]}\left(\log D_{0}\right) \rightarrow \Omega_{D_{0}}^{[p-1]}
$$

exists. Since $p>1$, Lemma25.10 implies

$$
H^{0}\left(D_{0}, \Omega_{D_{0}}^{[p-1]}\right)=0
$$

so that $\rho^{[p]}(\sigma)=0$. As observed in Remark 11.8 on page 32, this shows that $\sigma \in$ $H^{0}\left(X, \Omega_{X}^{[p]}\right)$, finishing the proof of Proposition 19.3 in case $\operatorname{dim} \pi\left(D_{0}\right)=0$.

25.F. Proof of Claim 25.3 if $\operatorname{dim} \pi\left(D_{0}\right)>0$. The proof of Claim 25.3 in case $\operatorname{dim} \pi\left(D_{0}\right)>0$ is at its core rather similar to the arguments of the preceding Section 25.E However, rather than applying the residue sequence directly to obtain a reflexive differential on $D_{0}$, we need to discuss the filtrations induced by relative differentials. Dealing with reflexive sheaves on singular spaces poses a few technical problems which will be discussed — and eventually overcome — in the following few subsections.

25.F.1. Relating Claim 25.3 to the reflexive restriction of $\sigma$. To prove Claim 25.3, we need to show that $\sigma \in H^{0}\left(X, \Omega_{X}^{[p]}\right)$. Since all sheaves in question are torsion-free, this may be checked on any open subset of $X$ which intersects $D_{0}$ non-trivially. To be more specific, let $X^{\circ} \subseteq X$ be the maximal open set where the pair $\left(X, D_{0}\right)$ is snc, and where the morphism $\psi$ is an snc morphism both of $(X, \emptyset)$ and of $\left(X, D_{0}\right)$. To prove Claim 25.3, it will then suffice to show that $\left.\sigma\right|_{X^{\circ}} \in H^{0}\left(X^{\circ}, \Omega_{X^{\circ}}^{p}\right)$.

We aim to study $\sigma$ by looking at its restriction $\left.\sigma\right|_{D_{0}^{\circ}}$, where $D_{0}^{\circ}:=D_{0} \cap X^{\circ}$. The restriction is governed by the following commutative diagram, whose first row is the standard residue sequence (11.1). The second row is the obvious restriction to $D_{0}^{\circ}$,

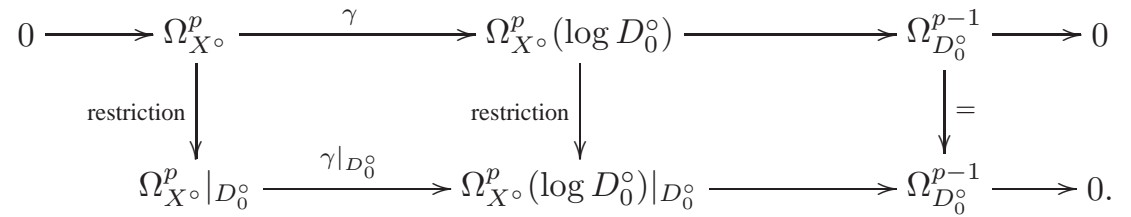

A quick diagram chase thus reveals that to show $\left.\sigma\right|_{X^{\circ}} \in H^{0}\left(X^{\circ}, \Omega_{X^{\circ}}^{p}\right)$, it suffices to show that the restriction of $\left.\sigma\right|_{D_{0}^{\circ}}$ comes from $\left.\Omega_{X^{\circ}}^{p}\right|_{D_{0}^{\circ}}$. More precisely, we see that to prove Claim 25.3 it suffices to show that

$$
\left.\sigma\right|_{D_{0}^{\circ}} \in \operatorname{Im}\left[\left.\gamma\right|_{D_{0}^{\circ}}: H^{0}\left(D_{0}^{\circ},\left.\Omega_{X^{\circ}}^{p}\right|_{D_{0}^{\circ}}\right) \rightarrow H^{0}\left(D_{0}^{\circ},\left.\Omega_{X^{\circ}}^{p}\left(\log D_{0}^{\circ}\right)\right|_{D_{0}^{\circ}}\right)\right] .
$$

Next, we aim to express the inclusion in 25.11.3 in terms of reflexive differentials which are globally defined along the divisor $D_{0}$, making the statement more amenable to the methods developed in Part

$$
\left.\left.\left(\left.\Omega_{X}^{[p]}\right|_{D_{0}} ^{* *}\right)\right|_{D_{0}^{\circ}} \simeq \Omega_{X^{\circ}}^{p}\right|_{D_{0}^{\circ}} \text { and }\left.\left.\left(\left.\Omega_{X}^{[p]}\left(\log D_{0}\right)\right|_{D_{0}} ^{* *}\right)\right|_{D_{0}^{\circ}} \simeq \Omega_{X^{\circ}}^{p}\left(\log D_{0}^{\circ}\right)\right|_{D_{0}^{\circ}} .
$$

Thus, if $\widetilde{\sigma}_{D_{0}} \in H^{0}\left(D_{0},\left.\Omega_{X}^{[p]}\left(\log D_{0}\right)\right|_{D_{0}} ^{* *}\right)$ denotes the image of $\left.\sigma\right|_{D_{0}}$ in the reflexive hull of $\left.\Omega_{X}^{[p]}\left(\log D_{0}\right)\right|_{D_{0}}$, then the inclusion in (25.11.3) will hold if we show that

$$
\widetilde{\sigma}_{D_{0}} \in \operatorname{Im}\left[H^{0}\left(D_{0},\left.\Omega_{X}^{[p]}\right|_{D_{0}} ^{* *}\right) \rightarrow H^{0}\left(D_{0},\left.\Omega_{X}^{[p]}\left(\log D_{0}\right)\right|_{D_{0}} ^{* *}\right)\right] .
$$

We will show more, namely, that $\widetilde{\sigma}_{D_{0}}$ is not only in the image of the sheaf $\left.\Omega_{X}^{[p]}\right|_{D_{0}} ^{* *}$, but that it is already in the image of the subsheaf $\left.\psi^{*} \Omega_{T}^{r}\right|_{D_{0}}$. The following lemma will be useful in the formulation of that claim. 
Lemma 25.12. The natural inclusions $\psi^{*} \Omega_{T}^{p} \hookrightarrow \Omega_{X}^{[p]} \hookrightarrow \Omega_{X}^{[p]}\left(\log D_{0}\right)$ yield a diagram of sheaves as follows,

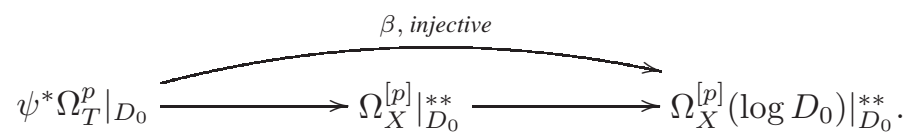

Proof. Assumption 25.9 allows to apply Theorem 10.6 from Page 27 (existence of relative differential sequences) to the sheaves $\Omega_{X}^{[p]}$ and $\Omega_{X}^{[p]}\left(\log D_{0}\right)$, obtaining a commutative diagram of injective sheaf morphisms,

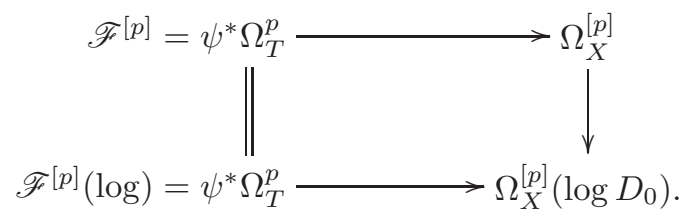

The diagram 25.12.1) is obtained by restricting (25.12.2) to $D_{0}$ and taking double duals. Injectivity of the map $\beta$ follows from a repeated application of Corollary 10.7 to the sheaf $\psi^{*} \Omega_{T}^{p}=\left.\mathscr{F}[p](\log )\right|_{D_{0}} ^{* *}$. This finishes the proof of Lemma 25.12

Returning to the proof of Claim 25.3, observe that Lemma 25.12 allows us to view $\left.\psi^{*} \Omega_{T}^{p}\right|_{D_{0}}$ as a subsheaf

$$
\left.\psi^{*} \Omega_{T}^{p}\right|_{D_{0}} \subseteq \operatorname{Im}\left[\left(\left.\Omega_{X}^{[p]}\right|_{D_{0}}\right)^{* *} \rightarrow\left(\left.\Omega_{X}^{[p]}\left(\log D_{0}\right)\right|_{D_{0}}\right)^{* *}\right] .
$$

With this notation, to prove the inclusion in 25.11.4), it is thus sufficient to prove the following claim.

Claim 25.13 (Proves 25.11.4) and hence Proposition 19.3 $n+1$ )). The section $\widetilde{\sigma}_{D_{0}}$ comes from $T$. More precisely, we claim that we have inclusions

$$
\widetilde{\sigma}_{D_{0}} \in H^{0}(D_{0}, \underbrace{\left.\psi^{*} \Omega_{T}^{p}\right|_{D_{0}}}_{=\left.\mathscr{F}[p](\log )\right|_{D_{0}^{* *}} ^{* *}}) \subseteq H^{0}\left(D_{0},\left(\left.\Omega_{X}^{[p]}\left(\log D_{0}\right)\right|_{D_{0}}\right)^{* *}\right) .
$$

25.F.2. Filtrations induced by relative differentials and their inclusions. Recall from Assumption 25.9 Theorem 10.6 and Corollary 10.7 that there exists a filtration of $\Omega_{X}^{[p]}\left(\log D_{0}\right)$,

$$
\Omega_{X}^{[p]}\left(\log D_{0}\right)=\mathscr{F}^{[0]}(\log ) \supseteq \mathscr{F}^{[1]}(\log ) \supseteq \cdots \supseteq \mathscr{F}^{[p]}(\log ) \supseteq\{0\}
$$

giving rise to exact sequences

$$
\left.\left.\left.0 \rightarrow \mathscr{F}^{[r+1]}(\log )\right|_{D_{0}} ^{* *} \rightarrow \mathscr{F}^{[r]}(\log )\right|_{D_{0}} ^{* *} \rightarrow \psi^{*} \Omega_{T}^{r} \otimes \Omega_{X / T}^{[p-r]}\left(\log D_{0}\right)\right|_{D_{0}} ^{* *} .
$$

Since $\psi^{*} \Omega_{T}^{p}$ is a trivial vector bundle, we see that to prove Claim 25.13 it is sufficient to prove the following.

Claim 25.14. For all numbers $q>0$, we have $H^{0}\left(D_{0},\left.\Omega_{X / T}^{[q]}\left(\log D_{0}\right)\right|_{D_{0}} ^{* *}\right)=0$. 
25.F.3. Proof of Claim 25.14 in case $q=1$. We argue by contradiction and assume that there exists a non-zero section $\tau \in H^{0}\left(D_{0},\left.\Omega_{X / T}^{[1]}\left(\log D_{0}\right)\right|_{D_{0}} ^{* *}\right)$.

We maintain the notation introduced in Section 25.F.1 If $t \in T$ is general, the section $\tau$ will then induce a non-zero section

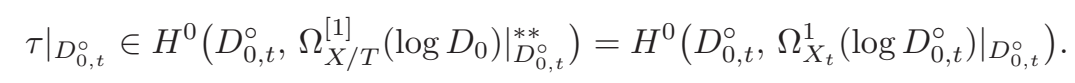

On the other hand, let $\beta$ be the composition of the following canonical morphisms

$$
\begin{aligned}
& H^{0}\left(X,\left.\Omega_{X / T}^{[1]}\left(\log D_{0}\right)\right|_{X_{t}} ^{* *}\right) \underset{\text { restr. to } X^{\circ}}{\simeq} H^{0}\left(X^{\circ}, \Omega_{X^{\circ} / T}^{1}\left(\log D_{0}^{\circ}\right)\right) \longrightarrow \\
& \underset{\text { restr. to } X_{t}^{\circ}}{\longrightarrow} H^{0}\left(X_{t}^{\circ},\left.\Omega_{X^{\circ} / T}^{1}\left(\log D_{0}^{\circ}\right)\right|_{X_{t}^{\circ}}\right) \underset{\psi_{X^{\circ}} \text { is snc }}{\simeq} H^{0}\left(X_{t}^{\circ}, \Omega_{X_{t}^{\circ}}^{1}\left(\log D_{0, t}^{\circ}\right)\right) \longrightarrow \\
& \underset{\text { restr. to } X_{t}^{\mathrm{o}}}{\stackrel{\simeq}{\longrightarrow}} H^{0}\left(X_{t}, \Omega_{X_{t}}^{[1]}\left(\log D_{0, t}\right)\right) \underset{\text { restr. to } D_{0, t}}{\longrightarrow} H^{0}\left(D_{0, t},\left.\Omega_{X_{t}}^{[1]}\left(\log D_{0, t}\right)\right|_{D_{0, t}}\right) \longrightarrow \\
& \underset{\text { refl. hull }}{\longrightarrow} H^{0}\left(D_{0, t},\left.\Omega_{X_{t}}^{[1]}\left(\log D_{0, t}\right)\right|_{D_{0, t}} ^{* *}\right) .
\end{aligned}
$$

Then a comparison with 25.14.1 immediately shows that $\left.\beta(\tau)\right|_{D_{0, t}^{\circ}} \neq 0$. In particular, we obtain that

$$
H^{0}\left(D_{0, t},\left.\Omega_{X_{t}}^{[1]}\left(\log D_{0, t}\right)\right|_{D_{0, t}} ^{* *}\right) \neq 0
$$

On the other hand, Theorem 12.2 on page 35 (description of Chern class by residue sequence) shows that there exists a smooth open subset $D_{0, t}^{\circ} \subseteq D_{0, t}$ with small complement,

$$
\operatorname{codim}_{D_{0, t}}\left(D_{0, t} \backslash D_{0, t}^{\circ \circ}\right) \geq 2 \text {, }
$$

and an exact sequence,

$$
\begin{aligned}
0 \rightarrow \underbrace{H^{0}\left(D_{0, t}^{\circ}, \Omega_{\left.D_{0, t}^{\circ}\right)}^{1}\right)}_{=: \mathrm{A}} & \rightarrow \underbrace{H^{0}\left(D_{0, t}^{\circ \circ},\left.\Omega_{X_{t}}^{[1]}\left(\log D_{0, t}\right)\right|_{\left.D_{0, t}^{\circ}\right)} ^{* *}\right)}_{=: \mathrm{B}} \rightarrow \\
& \rightarrow \underbrace{H^{0}\left(D_{0, t}^{\circ \circ}, \mathscr{O}_{\left.D_{0, t}^{\circ \circ}\right)}\right.}_{=: \mathrm{C}} \stackrel{\delta}{\rightarrow} H^{1}\left(D_{0, t}^{\circ \circ}, \Omega_{D_{0, t}^{\circ \circ}}^{1}\right) \rightarrow \cdots,
\end{aligned}
$$

where $\delta(m \cdot \mathbf{1})=c_{1}\left(\mathscr{O}_{D_{0, t}^{\circ \circ}}\left(m \cdot D_{0, t}^{\circ \circ}\right)\right)$, for $m$ sufficiently large and divisible. Observing that

$$
\begin{aligned}
& \mathrm{A} \simeq H^{0}\left(D_{0, t}, \Omega_{D_{0, t}}^{[1]}\right)=0 \\
& \mathrm{~B} \simeq H^{0}\left(D_{0, t},\left.\Omega_{X_{t}}^{[1]}\left(\log D_{0, t}\right)\right|_{D_{0, t}} ^{* *}\right) \neq 0 \\
& \mathrm{C} \simeq H^{0}\left(D_{0, t}, \mathscr{O}_{D_{0, t}}\right) \simeq \mathbb{C}
\end{aligned}
$$

The sequence 25.14.4 immediately implies that $c_{1}\left(\mathscr{O}_{D_{0, t}^{\circ \circ}}\left(m D_{0, t}^{\circ \circ}\right)\right)=0$. That, however, cannot be true, as the contraction $\left.\lambda\right|_{X_{t}}: X_{t} \rightarrow X_{\lambda, t}$ contracts the divisor $D_{0, t} \subset X_{t}$ to a point, so that Assertion A.12 of the Negativity Lemma A.1 implies that $D_{0, t}$ is actually $\mathbb{Q}$-anti-ample, relatively with respect to the contraction morphism $\left.\lambda\right|_{X_{t}}$. By the inequality (25.14.3), it is then also clear that $c_{1}\left(\mathscr{O}_{D_{0, t}^{\circ \circ}}\left(m D_{0, t}^{\circ \circ}\right)\right) \in H^{2}\left(D_{0, t}^{\circ \circ}, \mathbb{R}\right)$ cannot be zero. In fact, choose a complete curve $C \subset D_{0, t}^{\circ \circ}$ and observe that the restriction $\mathscr{O}_{D_{0, t}^{\circ \circ}}^{\circ}\left(m D_{0, t}^{\circ \circ}\right) \mid C$ is a negative line bundle. We obtain a contradiction which shows that the original assumption about the existence of a non-zero section $\tau$ was absurd. This completes the proof of Claim 25.14 in case $q=1$. 
25.F.4. Proof of Claim 25.14 in case $q>1$. Using Assumption 25.9 and applying the left-exact section functor $\Gamma$ to the residue sequence 11.711 constructed in Theorem 11.7 we obtain an exact sequence,

$$
0 \rightarrow \underbrace{H^{0}\left(D_{0}, \Omega_{D_{0} / T}^{[q]}\right)}_{=0 \text { by Lemma 25.11 }} \rightarrow H^{0}\left(D_{0},\left.\Omega_{X / T}^{[q]}\left(\log D_{0}\right)\right|_{D_{0}} ^{* *}\right) \rightarrow \underbrace{H^{0}\left(D_{0}, \Omega_{D_{0} / T}^{[q-1]}\right)}_{=0 \text { by Lemma [25.11 }},
$$

and Claim 25.14 follows immediately. This finishes the proof of Proposition 19.3 in case $\operatorname{dim} \pi\left(D_{0}\right)>0$.

\section{PART VII. Appendix}

\section{APPENDIX A. EFFECTIVE LINEAR COMBINATIONS OF EXCEPTIONAL DIVISORS}

The following Negativity Lemma is well-known to experts, and variants are found in the literature. Since the Negativity Lemma is central to our arguments, we reproduce a full proof here for the reader's convenience.

Lemma A.1 (Negativity Lemma for exceptional divisors, cf. [BCHM10, Lem. 3.6.2]). Let $\pi: \widetilde{X} \rightarrow X$ be a birational, projective and surjective morphism between irreducible and normal quasi-projective varieties.

(A.1.1) If $D$ is a $\pi$-exceptional $\mathbb{Q}$-divisor on $\widetilde{X}$ which is $\mathbb{Q}$-Cartier and $\pi$-anti-ample, then $D$ is effective, and $\operatorname{supp}(D)=E$.

(A.1.2) If $X$ is $\mathbb{Q}$-factorial, then there exists an effective and $\pi$-anti-ample Cartier divisor $D$ on $\widetilde{X}$ with $\operatorname{supp}(D)=E$. In particular, the $\pi$-exceptional set is of pure codimension one in $\widetilde{X}$.

(A.1.3) If $D \subset \widetilde{X}$ is any non-trivial effective $\mathbb{Q}$-Cartier divisor with $\operatorname{supp}(D) \subseteq E$, then $D$ is not $\pi$-nef.

Proof of A.1]Z. Since A.111 is local on $X$, we may assume without loss of generality that $X$ is affine, and that there exists a number $m \in \mathbb{N}$ such that the divisor $m D$ is integral, Cartier, and such that the linear system $|-m D|$ is relatively basepoint-free.

Decompose $D=D_{\text {pos }}-D_{\text {neg, }}$, where $D_{\text {pos }}$ and $D_{\text {neg }}$ are both effective, and do not share a common component. A section $\sigma \in H^{0}\left(\widetilde{X}, \mathscr{O}_{\widetilde{X}}(-m D)\right)$ is then seen as a rational function $f_{\sigma}$ on $\tilde{X}$ with prescribed zeros along $D_{\text {pos }}$, and possibly with poles of bounded order along $D_{\text {neg. }}$. It is, however, clear that $f_{\sigma}$ cannot have any poles at all: the function $f_{\sigma}$, which is certainly regular away from $E$, defines a function $g_{\sigma}$ in $X \backslash \pi(E)$. Since $\operatorname{codim}_{X} \pi(E) \geq 2$, the function $g_{\sigma}$ will extend to a function which is regular all over $X$, and whose pull-back necessarily agrees with $f_{\sigma}$. In summary, we obtain that the linear system $|-m D|$ has basepoints along $D_{\text {neg. }}$. It follows that $D_{\text {neg }}=0$.

It remains to show that $\operatorname{supp}(D)=E$. That, however, follows from the fact that the $\pi$-anti-ample divisor $D$ intersects every curve in $C \subset E$ negatively if the curve is mapped to a point in $X$.

Proof of (A.112). Let $D^{\prime} \subset \tilde{X}$ be any divisor which is $\pi$-anti-ample; $D^{\prime}$ exists because the morphism $\pi$ is assumed to be projective. By assumption, there exists a number $m$ such that $m$ times the cycle-theoretic image $\pi_{*} D^{\prime}$ is Cartier. The divisor $D:=m D^{\prime}-\pi^{*}\left(m \pi_{*} D^{\prime}\right)$ is then $\pi$-anti-ample and supported on $E$. Apply A.111 to conclude that $D$ is effective and that $\operatorname{supp}(D)=E$. 
Proof of (A.1,3]. Let $d:=\operatorname{dim} \pi(E)$. Choose general hypersurfaces $H_{1}, \ldots, H_{d} \subset X$ and $H_{d+1}, \ldots, H_{\operatorname{dim} X-2} \subset \widetilde{X}$. Further, set

$$
H:=\pi^{-1}\left(H_{1}\right) \cap \cdots \pi^{-1}\left(H_{d}\right) \cap H_{d+1} \cap \cdots \cap H_{\operatorname{dim} X-2} \subset \widetilde{X} .
$$

By Seidenberg's Theorem, [BS95, Thm. 1.7.1], the intersection $H$ is then a normal surface. Further, it follows from the construction that the cycle-theoretic intersection $D_{H}:=D \cap$

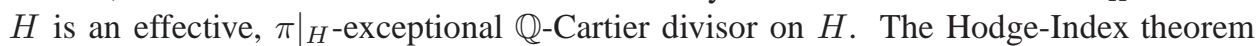
therefore asserts that $\left(D_{H}\right)^{2}<0$. It follows that there exists a curve $C \subseteq \operatorname{supp} D_{H} \subset$ $H \subset X$ which is contained in the $\pi$-exceptional set and intersects $D$ negatively, $D . C<0$. This completes the proof.

\section{APPENDIX B. FINITE GROUP ACTIONS ON COHERENT SHEAVES}

Let $G$ be a finite group acting on a normal variety $X$. In this appendix, we consider $G$-sheaves on $X$ and their associated push-forward sheaves on the quotient space. Some results presented here are well-known to experts. LemmaB.3 is contained for example in the unpublished preprint [Kol]. However, since we were not able to find published proofs of any of these result we decided to include them here in order to keep our exposition as self-contained as possible.

Definition B.1 ( $G$-sheaf and morphisms of $G$-sheaves). Let $G$ be a finite group acting on a normal variety $X$. If $g \in G$ is any element, we denote the associated automorphism of $X$ by $\phi_{g}$. A $G$-sheaf $\mathscr{F}$ on $X$ is a coherent sheaf of $\mathscr{O}_{X}$-modules such that for any open set $U \subseteq X$ is any open set, there exist natural push-forward morphisms

$$
\left(\phi_{g}\right)_{*}: \mathscr{F}(U) \rightarrow \mathscr{F}\left(\phi_{g}(U)\right)
$$

that satisfy the usual compatibility conditions. A morphism $\alpha: \mathscr{F} \rightarrow \mathscr{G}$ of $G$-sheaves is a sheaf morphism such that for any open set $U$ and any element $g \in G$, then there are commutative diagrams

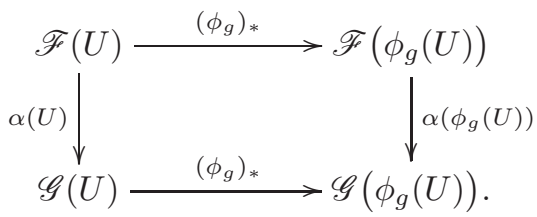

Definition B.2 (Invariant sheaves). If $G$ acts trivially on $X$, and if $\mathscr{F}$ is any $G$-sheaf, the associated sheaf of invariants, denoted $\mathscr{F}^{G}$, is the sheaf associated to the complete presheaf

$$
\mathscr{F}^{G}(U):=(\mathscr{F}(U))^{G}
$$

where $(\mathscr{F}(U))^{G}$ denotes the submodule of $G$-invariant elements of the $\mathscr{O}_{X}(U)$-module $\mathscr{F}(U)$.

In the remainder of the present Section B we consider the setup where $G$ acts on $X$, with quotient morphism $q: X \rightarrow X / G$. Let $\mathscr{G}$ be a coherent $G$-sheaf of $\mathscr{O}_{X}$-modules. Then, the push-forward $q_{*} \mathscr{G}$ is a $G$-sheaf on $X / G$ for the trivial $G$-action on $X / G$, and its associated sheaf of invariants will be denoted by $\left(q_{*} \mathscr{G}\right)^{G}$. The following lemmas collect fundamental properties of the functor $q_{*}(\cdot)^{G}$.

Lemma B.3 (Exactness Lemma). Let $G$ be a finite group acting on a normal variety $X$, and let $q: X \rightarrow X / G$ be the quotient morphism. Let $\mathscr{G}$ be a coherent $G$-sheaf of 
$\mathscr{O}_{X}$-modules. Then, the G-invariant push-forward $\left(q_{*} \mathscr{G}\right)^{G}$ is a coherent sheaf of $\mathscr{O}_{X / G^{-}}$ modules. Furthermore, if

$$
0 \rightarrow \mathscr{F} \rightarrow \mathscr{G} \rightarrow \mathscr{H} \rightarrow 0
$$

is a $G$-equivariant exact sequence of $\mathscr{O}_{X}$-modules, the induced sequence

$$
0 \rightarrow\left(q_{*} \mathscr{F}\right)^{G} \rightarrow\left(q_{*} \mathscr{G}\right)^{G} \rightarrow\left(q_{*} \mathscr{H}\right)^{G} \rightarrow 0
$$

is likewise exact.

Proof. The sequence (B.3.1) is clearly left-exact. For right-exactness, it follows from a classical result of Maschke [Mas1899] that any finite group in characteristic zero is linearly reductive. In other words, any finite-dimensional representation of $G$ splits as a direct sum of irreducible $G$-subrepresentations. It follows that for every $G$-representation $V$, there exists a Reynolds operator, i.e., a $G$-invariant projection $R: V \rightarrow V^{G}$, see for example, [Fog69. Sect. V-2]. It follows that $V^{G}$ is a direct summand of $V$.

So, if $\mathscr{G}$ is any coherent $G$-sheaf on $X$, it follows from the above that $\left(q_{*} \mathscr{G}\right)^{G}$ is a direct summand of the coherent sheaf $q_{*}(\mathscr{G})$ on $X / G$. Consequently, $\left(q_{*} \mathscr{G}\right)^{G}$ is likewise coherent.

Another consequence of the existence of the Reynolds operator is that for every $G$-equivariant map $\varphi: V \rightarrow W$ between (not necessarily finite-dimensional) $G$ representations, the induced map $\varphi^{G}: V^{G} \rightarrow W^{G}$ between the subspaces of invariants is still surjective. This shows right-exactness of (B.3.1) and implies the claim.

Lemma B.4 (Reflexivity Lemma). Let $G$ be a finite group, $X$ a normal $G$-variety, and $\mathscr{G}$ a reflexive coherent $G$-sheaf. Then, the $G$-invariant push-forward $\left(q_{*} \mathscr{G}\right)^{G}$ is also reflexive.

Proof. We have to show that $\left(q_{*} \mathscr{G}\right)^{G}$ is torsion-free and normal. Since $\mathscr{G}$ is torsion-free, $q_{*} \mathscr{G}$ is torsion-free, and hence $\left(q_{*} \mathscr{G}\right)^{G}$ is torsion-free as a subsheaf of $q_{*} \mathscr{G}$. To prove normality, let $U$ be an affine open subset of $X / G$ and $Z \subset U$ a closed subvariety of codimension at least 2 . Let

$$
s \in H^{0}\left(U \backslash Z,\left(q_{*} \mathscr{G}\right)^{G}\right)=H^{0}\left(q^{-1}(U) \backslash q^{-1}(Z), \mathscr{G}\right)^{G} .
$$

Since $q$ is finite, $q^{-1}(Z)$ has codimension at least 2 in $q^{-1}(U)$. Since $\mathscr{G}$ is reflexive, hence normal, the section $s$ extends to a $G$-invariant section of $\mathscr{G}$ over $q^{-1}(U)$.

Lemma B.5 (Splitting Lemma). Let $G$ be a finite group acting on a normal variety $X$ with quotient $q: X \rightarrow X / G$. Let

$$
0 \rightarrow \mathscr{H} \rightarrow \mathscr{F} \rightarrow \mathscr{G} \rightarrow 0
$$

be a $G$-equivariant exact sequence of locally free $G$-sheaves on $Y$. Then, the induced exact sequence

$$
0 \rightarrow\left(q_{*} \mathscr{H}\right)^{G} \rightarrow\left(q_{*} \mathscr{F}\right)^{G} \rightarrow\left(q_{*} \mathscr{G}\right)^{G} \rightarrow 0
$$

is locally split in the analytic topology.

Proof. As shown in Figure 6 on the facing page, let $z \in X / G$ be any point and $x \in q^{-1}(z)$ any preimage point, with isotropy group $G_{x}$. By the holomorphic slice theorem, cf. [Hol61, Hilfssatz 1] or [Hei91, Sect. 5.5], there exists an open Stein neighbourhood $U=U(z) \subseteq$ $X / G$ and an open $G_{x}$-invariant Stein neighbourhood $V=V(x) \subseteq X$ such that $q^{-1}(U)$ is $G$-equivariantly biholomorphic to the twisted product

$$
G \times_{G_{x}} V:=(G \times V) / G_{x},
$$




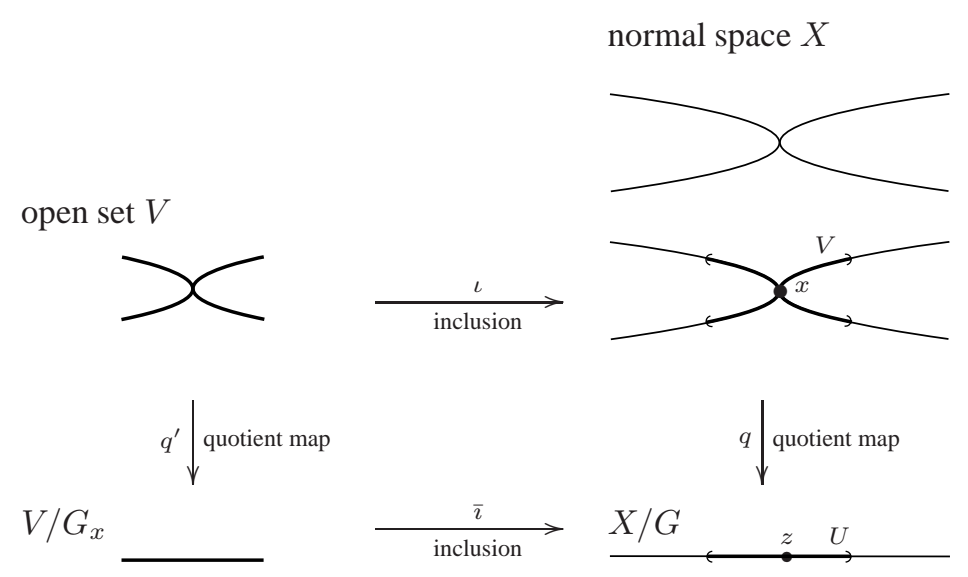

FIGURE 6. Setup in the proof of the Splitting Lemma B.4

where $G_{x}$ acts on $G \times V$ as

$$
\begin{array}{ccc}
G_{x} \times(G \times V) & \rightarrow & G \times V \\
(h,(g, v)) & \mapsto & \left(g h^{-1}, h \cdot v\right) .
\end{array}
$$

Let $q^{\prime}: V \rightarrow V / G_{x}$ denote the quotient of $V$ by $G_{x}$. Observe then that the inclusion $\imath: V \hookrightarrow q^{-1}(U)$ induces a biholomorphic map

$$
\bar{\imath}: V / G_{x} \stackrel{\simeq}{\longrightarrow} U=q^{-1}(U) / G .
$$

Shrinking $U$, if necessary, we may assume that the sequence $\overline{B .5 .1}$ is split on $V$ with splitting $s:\left.\left.\mathscr{G}\right|_{V} \rightarrow \mathscr{F}\right|_{V}$. By averaging $s$ over $G_{x}$ we obtain a sheaf morphism $\bar{s}$ : $\left(q_{*}^{\prime}\left(\left.\mathscr{G}\right|_{V}\right)\right)^{G_{x}} \rightarrow\left(q_{*}^{\prime}\left(\left.\mathscr{F}\right|_{V}\right)\right)^{G_{x}}$ that splits the exact sequence

$$
0 \rightarrow\left(q_{*}^{\prime}\left(\left.\mathscr{H}\right|_{V}\right)\right)^{G_{x}} \rightarrow\left(q_{*}^{\prime}\left(\left.\mathscr{F}\right|_{V}\right)\right)^{G_{x}} \rightarrow\left(q_{*}^{\prime}\left(\left.\mathscr{G}\right|_{V}\right)\right)^{G_{x}} \rightarrow 0 .
$$

Finally we notice that for any coherent $G$-sheaf $\mathscr{S}$ on $q^{-1}(U)$, the inclusion $\imath$ induces a canonical isomorphism

$$
\phi_{\mathscr{S}}: \bar{\imath}^{*}\left(q_{*} \mathscr{S}\right)^{G} \stackrel{\simeq}{\longrightarrow}\left(q_{*}^{\prime}\left(\left.\mathscr{S}\right|_{V}\right)\right)^{G_{x}} .
$$

Applying this observation to $\mathscr{F}$ and $\mathscr{G}$, we obtain a commutative diagram

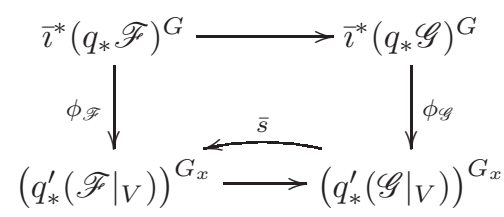

The map $\phi_{\mathscr{F}} \circ \bar{s} \circ \phi_{\mathscr{G}}$ then is the desired splitting.

Lemma B.6 (Restriction Lemma). Let $G$ be a finite group, $X$ a normal $G$-variety, and $\mathscr{F}$ locally free coherent $G$-sheaf on $X$. Let $q: X \rightarrow X / G$ be the quotient map, and let $\Delta$ be a normal $G$-invariant subvariety of $X$ with image $D=q(\Delta)$. Then, we have a canonical surjection

$$
\left(\left.\left(q_{*} \mathscr{F}\right)^{G}\right|_{D}\right)^{* *} \rightarrow\left(\left.q\right|_{\Delta}\right)_{*}\left(\left.\mathscr{F}\right|_{\Delta}\right)^{G} .
$$


Proof. Let $\imath: \Delta \hookrightarrow X$ denote the inclusion. Clearly, the restriction morphism $\mathscr{F} \rightarrow \imath_{*}\left(\left.\mathscr{F}\right|_{\Delta}\right)$ is $G$-equivariant. Since $q$ is finite, we obtain a surjection $q_{*}(\mathscr{F}) \rightarrow$ $q_{*}\left(\imath_{*}\left(\left.\mathscr{F}\right|_{\Delta}\right)\right)$. The Exactness Lemma B.3 implies that the induced map of invariants $\left(q_{*} \mathscr{F}\right)^{G} \rightarrow q_{*}\left(\imath_{*}\left(\left.\mathscr{F}\right|_{\Delta}\right)\right)^{G}$ is still surjective. This morphism stays surjective after restriction to $D$, i.e. we obtain a surjection

$$
\varphi:\left.\left.\left(q_{*} \mathscr{F}\right)^{G}\right|_{D} \rightarrow q_{*}\left(\imath_{*}\left(\left.\mathscr{F}\right|_{\Delta}\right)\right)^{G}\right|_{D}=\left(\left.q\right|_{\Delta}\right)_{*}\left(\left.\mathscr{F}\right|_{\Delta}\right)^{G} .
$$

Since the restriction $\left.\mathscr{F}\right|_{\Delta}$ is locally free and $\Delta$ is normal by assumption, the Reflexivity Lemma B.4 implies that $\left(\left.q\right|_{\Delta}\right)_{*}\left(\left.\mathscr{F}\right|_{\Delta}\right)^{G}$ is reflexive and hence torsion-free. As a consequence $\varphi$ factors over the natural map $\left.\left(q_{*} \mathscr{F}\right)^{G}\right|_{D} \rightarrow\left(\left.\left(q_{*} \mathscr{F}\right)^{G}\right|_{D}\right)^{* *}$. This shows the claim.

\section{APPENDiX C. THE FOUR-LEMMAS FOR VECTOR SPACES}

For the reader's convenience, we recall the elementary 4-Lemmas from commutative algebra.

Lemma C.1 (Four-Lemma for injectivity, [ML95, XII Lem. 3.1(i)]). Consider a commutative diagram of linear maps between vector spaces, as follows

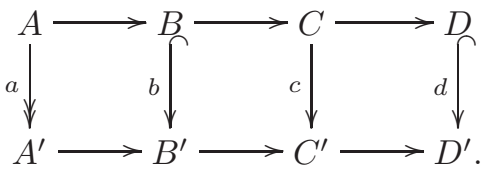

If the horizontal sequences are exact, then $c$ is injective.

Lemma C.2 (Four-Lemma for surjectivity, [ML95, XII Lem. 3.1(ii)]). Consider a commutative diagram of linear maps between vector spaces, as follows

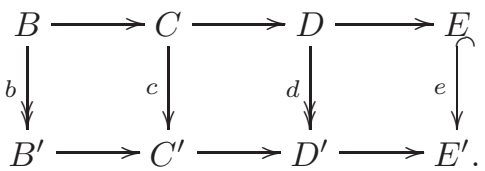

If the horizontal sequences are exact, then $c$ is surjective.

\section{REFERENCES}

[Bar78] D. BARLET: Le faisceau $\omega_{X}$ sur un espace analytique $X$ de dimension pure, Fonctions de plusieurs variables complexes III (Sém. François Norguet, 1975-1977), pp. 187--204, Lecture Notes in Math., 670, Springer, Berlin, 1978. MR0521919 (80i:32037)

[Bau07] J. BAUM: Aufblasungen und Desingularisierungen, Staatsexamensarbeit, Universität zu Köln. Available on the internet at http://home.mathematik.uni-freiburg.de/kebekus/teaching/thesis-de.html, 2007.

[BS95] M. C. BELTRAMETTI AND A. J. SOMmESE: The adjunction theory of complex projective varieties, de Gruyter Expositions in Mathematics, vol. 16, Walter de Gruyter \& Co., Berlin, 1995. 96f:14004

[BCHM10] C. BIRKAR, P. CASCINI, C. D. HACON, AND J. MCKERNAN: Existence of minimal models for varieties of log general type, Journal of the AMS 23 (2010), 405-468, DOI:10.1090/S0894-0347-09-00649-3

[Cam04] F. CAMPANA: Orbifolds, special varieties and classification theory, Ann. Inst. Fourier (Grenoble) 54 (2004), no. 3, 499-630. MR2097416 (2006c:14013)

[Car85] J. A. CARLson: Polyhedral resolutions of algebraic varieties, Trans. Amer. Math. Soc. 292 (1985), no. 2, 595-612. MR808740 (87i:14008)

[Cor07] A. CoRTi ET AL.: Flips for 3-folds and 4-folds, Oxford Lecture Series in Mathematics and its Applications, vol. 35, Oxford University Press, Oxford, 2007. MR2352762 (2008j:14031) 
[Del70] P. Deligne: Équations différentielles à points singuliers réguliers, Springer-Verlag, Berlin, 1970, Lecture Notes in Mathematics, Vol. 163. 54 \#5232

[DB81] P. Du BoIs: Complexe de de Rham filtré d'une variété singulière, Bull. Soc. Math. France 109 (1981), no. 1, 41-81. MR613848 (82j:14006)

[DBJ74] P. DU BOIS AND P. JARRAUD: Une propriété de commutation au changement de base des images directes supérieures du faisceau structural, C. R. Acad. Sci. Paris Sér. A 279 (1974), 745-747. MR0376678 (51 \#12853)

[dJS04] A. J. DE Jong AND J. STARR: Cubic fourfolds and spaces of rational curves, Illinois J. Math. 48 (2004), no. 2, 415-450. MR2085418 (2006e:14007)

[EV82] H. Esnault And E. Viehweg: Revêtements cycliques, in: Algebraic threefolds (Varenna, 1981). Springer-Verlag, Berlin, 1982, Lecture Notes in Mathematics, Vol. 947, pp. 241-250. MR0672621 (84m:14015)

[EV90] H. ESNAULT AND E. VIEHWEG: Effective bounds for semipositive sheaves and for the height of points on curves over complex function fields, Compositio Math. 76 (1990), no. 1-2, 69-85, Algebraic geometry (Berlin, 1988). MR1078858 (91m:14038)

[EV92] H. Esnault AND E. Viehweg: Lectures on vanishing theorems, DMV Seminar, vol. 20, Birkhäuser Verlag, Basel, 1992. MR1193913 (94a:14017)

[FGI $\left.{ }^{+} 05\right]$ B. Fantechi, L. Göttsche, L. Illusie, S. L. Kleiman, N. Nitsure, and A. Vistoli: Fundamental algebraic geometry, Mathematical Surveys and Monographs, vol. 123, American Mathematical Society, Providence, RI, 2005, Grothendieck's FGA explained. MR2222646 (2007f:14001)

[Fle88] H. FlenNer: Extendability of differential forms on nonisolated singularities, Invent. Math. 94 (1988), no. 2, 317-326. MR958835 (89j:14001)

[Fog69] J. FogarTY: Invariant theory, W. A. Benjamin, Inc., New York-Amsterdam, 1969. MR0240104 (39 \#1458)

[God73] R. Godement: Topologie algébrique et théorie des faisceaux, Hermann, Paris, 1973, Troisième édition revue et corrigée, Publications de l'Institut de Mathématique de l'Université de Strasbourg, XIII, Actualités Scientifiques et Industrielles, No. 1252. MR0345092 (49 \#9831)

[Gra72] H. GRAUERT: Über die Deformation isolierter Singularitäten analytischer Mengen, Invent. Math. 15 (1972), 171-198. MR0293127 (45 \#2206)

[GR70] H. GRAUERT AND O. RIEMENSCHNEIDER: Verschwindungssätze für analytische Kohomologiegruppen auf komplexen Räumen, Invent. Math. 11 (1970), 263-292. MR0302938 (46 \#2081)

[GKK10] D. GReb, S. KebeKUs, AND S. J. KovÁcs: Extension theorems for differential forms, and Bogomolov-Sommese vanishing on log canonical varieties, Compos. Math. 146 (2010), 193-219, DOI:10.1112/S0010437X09004321

[GLS07] G.-M. GReuel, C. Lossen, And E. Shustin: Introduction to singularities and deformations, Springer Monographs in Mathematics, Springer, Berlin, 2007. MR2290112 (2008b:32013)

[Gre80] G.-M. GReUEL: Dualität in der lokalen Kohomologie isolierter Singularitäten, Math. Ann. 250 (1980), no. 2, 157-173. MR582515 (82e:32009)

[Gro60] A. GROTHENDIECK: Éléments de géométrie algébrique. I. Le langage des schémas, Inst. Hautes Études Sci. Publ. Math. (1960), no. 4, 228. MR0217083 (36 \#177a)

[Gro71] A. Grothendieck: Revêtements étales et groupe fondamental, Springer-Verlag, Berlin, 1971, Séminaire de Géométrie Algébrique du Bois Marie 1960-1961 (SGA 1), Dirigé par Alexandre Grothendieck. Augmenté de deux exposés de M. Raynaud, Lecture Notes in Mathematics, Vol. 224. MR0354651 (50 \#7129)

[GNPP88] F. Guillén, V. Navarro Aznar, P. Pascual Gainza, and F. Puerta: Hyperrésolutions cubiques et descente cohomologique, Lecture Notes in Mathematics, vol. 1335, Springer-Verlag, Berlin, 1988, Papers from the Seminar on Hodge-Deligne Theory held in Barcelona, 1982. MR972983 (90a:14024)

[HK10] C. D. HACON AND S. J. KovÁCS: Classification of higher dimensional algebraic varieties, Oberwolfach Seminars, Birkhäuser Boston, Boston, MA, 2010.

[HM07] C. D. HACON AND J. MCKeRnAn: On Shokurov's rational connectedness conjecture, Duke Math. J. 138 (2007), no. 1, 119-136. MR2309156 (2008f:14030)

[Har77] R. HARTShorne: Algebraic geometry, Springer-Verlag, New York, 1977, Graduate Texts in Mathematics, No. 52. MR0463157 (57 \#3116)

[HM89] H. HAUSER AND G. MÜLlER: The trivial locus of an analytic map germ, Ann. Inst. Fourier (Grenoble) 39 (1989), no. 4, 831-844. MR1036334 (91m:32035)

[Hei91] P. HeInZneR: Geometric invariant theory on Stein spaces, Math. Ann. 289 (1991), no. 4, 631-662. MR1103041 (92j:32116) 
[Hir62] H. Hironaka: On resolution of singularities (characteristic zero), Proc. Int. Cong. Math., 1962, pp. 507-521.

[Hoc75] M. Hochster: The Zariski-Lipman conjecture for homogeneous complete intersections, Proc. Amer. Math. Soc. 49 (1975), 261-262. MR0360585 (50 \#13033)

[Hol61] H. Holmann: Quotienten komplexer Räume, Math. Ann. 142 (1960/1961), 407-440. MR0120665 (22\#11414)

[HL97] D. HUYBRECHTS AND M. LEHN: The geometry of moduli spaces of sheaves, Aspects of Mathematics, E31, Friedr. Vieweg \& Sohn, Braunschweig, 1997. MR1450870 (98g:14012)

[JK09a] K. JABBUSCH AND S. KebeKUS: Families over special base manifolds and a conjecture of Campana, appeared electronically Math. Zeitschrift. To appear in print. DOI:10.1007/s00209-010-0758-6. arXiv:0905.1746. May 2009.

[JK09b] K. JabBusCh AND S. Kebenus: Positive sheaves of differentials on coarse moduli spaces, to appear in Ann. Inst. Fourier (Grenoble). arXiv:0904.2445 April 2009.

[Kaw88] Y. KaWAMATA: Crepant blowing-up of 3-dimensional canonical singularities and its application to degenerations of surfaces, Ann. of Math. (2) 127 (1988), no. 1, 93-163. MR924674 (89d:14023)

[KK07] S. KEBEKUS AND S. J. KovÁcs: The structure of surfaces mapping to the moduli stack of canonically polarized varieties, preprint arXiv:0707.2054 July 2007.

[KK08] S. KEBEKUS AND S. J. KovÁCs: Families of canonically polarized varieties over surfaces, Invent. Math. 172 (2008), no. 3, 657-682, DOI:10.1007/s00222-008-0128-8 MR2393082

[KK10a] S. KeBeKUS AND S. J. KovÁCS: The structure of surfaces and threefolds mapping to the moduli stack of canonically polarized varieties, Duke Math. J. 155 (2010), no. 1, 1-33. MR2730371. arXiv:0812.2305

[Kol] J. KOLLÁR: Algebraic groups acting on schemes, Undated, unfinished manuscript. Available on the author's website at www.math.princeton.edu/ kollar

[Kol96] J. Kollár: Rational curves on algebraic varieties, Ergebnisse der Mathematik und ihrer Grenzgebiete. 3. Folge. A Series of Modern Surveys in Mathematics, vol. 32, Springer-Verlag, Berlin, 1996. MR1440180 (98c:14001)

[Kol07] J. Kollár: Lectures on resolution of singularities, Annals of Mathematics Studies, vol. 166, Princeton University Press, Princeton, NJ, 2007. MR2289519

[KK10b] J. Kollár AND S. J. KovÁcs: Log canonical singularities are Du Bois, JAMS (2010), 23 pages, published electronically. DOI:10.1090/S0894-0347-10-00663-6

[KM98] J. KollÁR AND S. MORI: Birational geometry of algebraic varieties, Cambridge Tracts in Mathematics, vol. 134, Cambridge University Press, Cambridge, 1998, With the collaboration of C. H. Clemens and A. Corti, Translated from the 1998 Japanese original. 2000b:14018

[Ko192] J. Kollár ET AL.: Flips and abundance for algebraic threefolds, Société Mathématique de France, Paris, 1992, Papers from the Second Summer Seminar on Algebraic Geometry held at the University of Utah, Salt Lake City, Utah, August 1991, Astérisque No. 211 (1992). MR1225842 (94f:14013)

[KS09] S. J. KovÁCS AND K. SCHWEDE: Hodge theory meets the minimal model program: a survey of log canonical and Du Bois singularities, preprint, 2009. arXiv:0909.0993v1

[Lau73] H. B. LAUfER: Taut two-dimensional singularities, Math. Ann. 205 (1973), 131-164. MR0333238 (48 \#11563)

[Lip65] J. Lipman: Free derivation modules on algebraic varieties, Amer. J. Math. 87 (1965), 874-898. MR0186672 (32 \#4130)

[Loj64] S. Lojasiewicz: Triangulation of semi-analytic sets, Ann. Scuola Norm. Sup. Pisa (3) 18 (1964), 449-474. MR0173265 (30 \#3478)

[ML95] S. Mac Lane: Homology, Classics in Mathematics, Springer-Verlag, Berlin, 1995, Reprint of the 1975 edition. MR1344215 (96d:18001)

[Mas1899] H. MASchKe: Beweis des Satzes, dass diejenigen endlichen linearen Substitutionsgruppen, in welchen einige durchgehends verschwindende Coefficienten auftreten, intransitiv sind, Math. Ann. 52 (1899), no. 2-3, 363-368. MR1511061

[Nam01] Y. NAmiKaWA: Extension of 2-forms and symplectic varieties, J. Reine Angew. Math. 539 (2001), 123-147. MR1863856 (2002i:32011)

[OSS80] C. OKONEK, M. SCHNEIDER, AND H. SPINDLER: Vector bundles on complex projective spaces, Progress in Mathematics, vol. 3, Birkhäuser Boston, Mass., 1980. MR561910 (81b:14001)

[PS08] C. A. M. Peters And J. H. M. Steenbrink: Mixed Hodge structures, Ergebnisse der Mathematik und ihrer Grenzgebiete. 3. Folge. A Series of Modern Surveys in Mathematics [Results in Mathematics and Related Areas. 3rd Series. A Series of Modern Surveys in Mathematics], vol. 52, Springer-Verlag, Berlin, 2008. MR2393625 
[Pri67] D. PRILL: Local classification of quotients of complex manifolds by discontinuous groups, Duke Math. J. 34 (1967), 375-386. MR0210944 (35 \#1829)

[Rei80] M. ReID: Canonical 3-folds, Algebraic Geometry Angers 1979 (Alphen aan den Rijn) (A. Beauville, ed.), Sijthoff \& Noordhoff, 1980.

[Rei87] M. REID: Young person's guide to canonical singularities, Algebraic geometry, Bowdoin, 1985 (Brunswick, Maine, 1985), Proc. Sympos. Pure Math., vol. 46, Amer. Math. Soc., Providence, RI, 1987, pp. 345-414. MR927963 (89b:14016)

[SS72] G. SCHEJA AND U. STORCH: Differentielle Eigenschaften der Lokalisierungen analytischer Algebren, Math. Ann. 197 (1972), 137-170. MR0306172 (46 \#5299)

[Sei50] A. SeIDEnBERG: The hyperplane sections of normal varieties, Trans. Amer. Math. Soc. 69 (1950), 357-386. MR0037548 (12,279a)

[Sha94] I. R. ShAFAREVICH: Basic algebraic geometry. 1, second ed., Springer-Verlag, Berlin, 1994, Varieties in projective space, Translated from the 1988 Russian edition and with notes by Miles Reid. MR1328833 (95m:14001)

[SvS85] J. STEENBRINK AND D. VAN STRATEN: Extendability of holomorphic differential forms near isolated hypersurface singularities, Abh. Math. Sem. Univ. Hamburg 55 (1985), 97-110. MR831521 (87j:32025)

[Ste85] J. H. M. SteEnbrink: Vanishing theorems on singular spaces, Astérisque (1985), no. 130, 330341, Differential systems and singularities (Luminy, 1983). MR804061 (87j:14026)

[Sza94] E. Szabó: Divisorial log terminal singularities, J. Math. Sci. Univ. Tokyo 1 (1994), no. 3, 631-639. MR1322695 (96f:14019)

[Tei77] B. TEISSIER: The hunting of invariants in the geometry of discriminants, Real and complex singularities (Proc. Ninth Nordic Summer School/NAVF Sympos. Math., Oslo, 1976), Sijthoff and Noordhoff, Alphen aan den Rijn, 1977, pp. 565-678. MR0568901 (58 \#27964)

[Ver76] J.-L. VERDIER: Stratifications de Whitney et théorème de Bertini-Sard, Invent. Math. 36 (1976), 295-312. MR0481096 (58 \#1242)

[Vie10] E. VIEHWEG: Compactifications of smooth families and of moduli spaces of polarized manifolds, Ann. of Math. (2) 172 (2010), no. 2, 809-910. arXiv:math/0605093 MR2680483

[VZ02] E. VIEHWEG AND K. ZUO: Base spaces of non-isotrivial families of smooth minimal models, Complex geometry (Göttingen, 2000), Springer, Berlin, 2002, pp. 279-328. MR1922109 (2003h:14019)

[Wah85] J. M. WAHL: A characterization of quasihomogeneous Gorenstein surface singularities, Compositio Math. 55 (1985), no. 3, 269-288. MR799816 (87e:32013)

\section{LIST OF FIGURES}

1 A special case of the Pull-back theorem 4.3.

2 Two morphisms for which the assumptions of Theorem 15.1 hold 42

3 A three-dimensional example where Assumption 17.8 holds 47

4 Situation after projection to $\pi\left(E_{0}\right)$

5 Strong log resolution of an isolated klt surface singularity 52

6 Setup in the proof of the Splitting Lemma B.4 67

\section{LIST OF TABLES}

1 Lexicographical ordering of dimensions and codimensions 45

2 Long exact cohomology sequences for relative differentials 51 
3 Overview of the induction used to prove Theorem 1.5.

Daniel Greb, Mathematisches Institut, Albert-Ludwigs-Universität Freiburg, EckerSTRASSE 1, 79104 FREIBURG IM BREISGAU, GERMANY

E-mail address: daniel.greb@math.uni-freiburg.de

URL: http://home.mathematik.uni-freiburg.de/dgreb

Stefan Kebekus, Mathematisches Institut, Albert-Ludwigs-Universität Freiburg, EcKERSTRASSE 1, 79104 FREIBURG IM BREISGAU, GERMANY

E-mail address: stefan.kebekus@math.uni-freiburg.de

$U R L:$ http://home.mathematik.uni-freiburg.de/kebekus

Sándor Kovács, University of Washington, Department of Mathematics, Box 354350 , SEATTLE, WA 98195 , U.S.A.

E-mail address: kovacs@math.washington.edu

$U R L:$ http://www.math.washington.edu/ kovacs

Thomas Peternell, Institut für Mathematik, Universität Bayreuth, 95440 Bayreuth, GERMANY

E-mail address: thomas.peternelleuni-bayreuth.de

URL: http://btm8×5.mat.uni-bayreuth.de/mathe1 